\title{
Samarium(II)-mediated linker cleavage-cyclization in fluorous synthesis: reactions of samarium enolates
}

\author{
Karen M. James, ${ }^{a}$ Nigel Willetts ${ }^{b}$ and David J. Procter ${ }^{a}{ }^{*}$ \\ ${ }^{a}$ The School of Chemistry, The University of Manchester, Oxford Road, M13 9PL, UK. \\ ${ }^{b}$ Syngenta, Jealott's Hill International Research Centre, Bracknell, Berkshire, RG42 6EY, \\ UK.
}

Supporting Information

Table of Contents

Pages

General considerations

S2

Experimental

S3

X-ray crystallography data for $\mathbf{8}$

S28

${ }^{1} \mathrm{H}$ and ${ }^{13} \mathrm{C}$ spectra

S33 


\section{General Considerations}

All experiments were performed under an atmosphere of $\mathrm{N}_{2}$, using anhydrous solvents, unless stated otherwise. Reactions were carried out using oven-dried glassware. THF was distilled from sodium/benzophenone, $\mathrm{CH}_{2} \mathrm{Cl}_{2}$ was distilled from $\mathrm{CaH}_{2}, \mathrm{Et}_{2} \mathrm{O}$ was distilled from $\mathrm{CaH}_{2},{ }^{\mathrm{i}} \mathrm{PrNH}_{2}$ was distilled from $\mathrm{CaH}_{2}$. $\mathrm{Et}_{3} \mathrm{~N}$ was distilled from $\mathrm{CaH}_{2}$ and stored over $\mathrm{KOH}$ and under $\mathrm{N}_{2}$. DMSO was distilled from $\mathrm{CaH}_{2}$ and stored over molecular sieves and under $\mathrm{N}_{2}$.

${ }^{1} \mathrm{H}$ NMR and ${ }^{13} \mathrm{C}$ NMR were recorded on a Fourier transform spectrometer, with chemical shift values being reported in ppm relative to residual chloroform $\left(\delta_{\mathrm{H}}=7.27\right.$ or $\left.\delta_{\mathrm{C}}=77.2\right)$ as internal standard unless otherwise stated. NMR signals were assigned using DEPT-135, HMQC and COSY spectra. All coupling constants $(J)$ are reported in Hertz $(\mathrm{Hz})$. Mass spectra and microanalyses were recorded at the University of Manchester. IR spectra were recorded using a FT/IR spectrometer.

Column chromatography was carried out using silica gel 60 and FluoroFlash silica. Aluminium backed plates, pre-coated with silica gel $60\left(\mathrm{UV}_{254}\right)$ were used for thin layer chromatography and were visualized by UV or staining with alkali $\mathrm{KMnO}_{4}$. 


\section{Experimental}

\section{5-Bromo-3-(2-vinylsulfonylethyl)-3-(3,3,4,4,5,5,6,6,7,7,8,8,9,9,10,10,10- heptadecafluorodecylsulfonyl)-1-propyl-1,3-dihydroindol-2-one (5)}

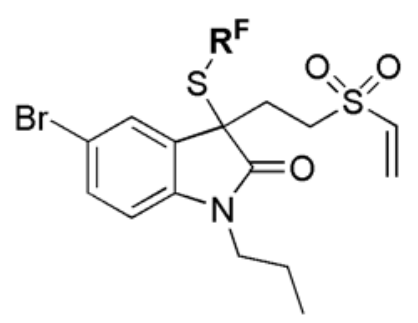

To a solution of 5-bromo-3-(3,3,4,4,5,5,6,6,7,7,8,8,9,9,10,10,10heptadecafluorodecylsulfanyl)-1-propyl-1,3-dihydroindol-2-one (0.207 g, $0.283 \mathrm{mmol}, 1$ eq) in $\mathrm{CH}_{2} \mathrm{Cl}_{2}(26 \mathrm{ml})$ was added DBU $(0.106 \mathrm{ml}, 0.708 \mathrm{mmol}, 2.5 \mathrm{eq})$ and divinyl sulfone $(0.040 \mathrm{ml}, 0.368 \mathrm{mmol}, 1.3 \mathrm{eq})$ at room temperature and the reaction allowed to stir for 18 hours. The reaction mixture was then quenched with aqueous saturated $\mathrm{NH}_{4} \mathrm{Cl}$ $(10 \mathrm{ml})$ and the organic layer dried $\left(\mathrm{MgSO}_{4}\right)$, filtered and concentrated in vacuo to give the crude product as a yellow solid which was purified by fluorous chromatography to give 5-bromo-3-(2-vinylsulfonylethyl)-3-(3,3,4,4,5,5,6,6,7,7,8,8,9,9,10,10,10heptadecafluorodecylsulfonyl)-1-propyl-1,3-dihydroindol-2-one 5 (0.196 g, $0.231 \mathrm{mmol}$, $81 \%$ ) as a white solid (mpt $92.1-93.6^{\circ} \mathrm{C}$ recrystallized from $\mathrm{MeOH}$ ).

${ }^{1} \mathrm{H}$ NMR $\left(500 \mathrm{MHz}, \mathrm{CDCl}_{3}\right) \delta 0.96\left(3 \mathrm{H}, \mathrm{t}, \mathrm{J}=7.5 \mathrm{~Hz}, \mathrm{CH}_{3}\right), 1.65-1.72\left(2 \mathrm{H}, \mathrm{m}, \mathrm{CH}_{2} \mathrm{CH}_{3}\right)$, 2.15-2.26 (2H, m, $\mathrm{CH}_{2} \mathrm{CF}_{2}$ ), 2.39-2.52 (2H, m, $\left.\mathrm{CH}_{2} \mathrm{CS}\right), 2.62-2.68\left(1 \mathrm{H}, \mathrm{m}, 1 \mathrm{H}\right.$ of $\left.\mathrm{CH}_{2} \mathrm{~S}\right)$, 2.70-2.76 $\left(1 \mathrm{H}, \mathrm{m}, 1 \mathrm{H}\right.$ of $\left.\mathrm{CH}_{2} \mathrm{~S}\right), 2.89-2.92\left(2 \mathrm{H}, \mathrm{m}, \mathrm{CH}_{2} \mathrm{SO}_{2}\right), 3.60-3.65(1 \mathrm{H}, \mathrm{m}, 1 \mathrm{H}$ of $\left.\mathrm{CH}_{2} \mathrm{~N}\right), 3.68-3.74\left(1 \mathrm{H}, \mathrm{m}, 1 \mathrm{H}\right.$ of $\left.\mathrm{CH}_{2} \mathrm{~N}\right), 6.23\left(1 \mathrm{H}, \mathrm{d}, \mathrm{J}=9.8 \mathrm{~Hz}, 1 \mathrm{H}\right.$ of $\left.\mathrm{CH}_{2}=\mathrm{CH}\right), 6.46$ $\left(1 \mathrm{H}, \mathrm{d}, \mathrm{J}=16.6 \mathrm{~Hz}, 1 \mathrm{H}\right.$ of $\left.\mathrm{CH}_{2}=\mathrm{CH}\right), 6.59\left(1 \mathrm{H}, \mathrm{dd}, \mathrm{J}=9.8,16.6 \mathrm{~Hz}, \mathrm{CH}=\mathrm{CH}_{2}\right), 6.79(1 \mathrm{H}$, $\mathrm{d}, \mathrm{J}=8.4 \mathrm{~Hz}, \operatorname{ArCH}), 7.44(1 \mathrm{H}, \mathrm{d}, \mathrm{J}=1.9 \mathrm{~Hz}, \operatorname{ArCH}), 7.50(1 \mathrm{H}, \mathrm{dd}, \mathrm{J}=1.9,8.4 \mathrm{~Hz}$, $\mathrm{ArCH})$.

${ }^{13} \mathrm{C}$ NMR (126 MHz, $\left.\mathrm{CDCl}_{3}\right) \delta 11.4\left(\mathrm{CH}_{3}\right), 19.8\left(\mathrm{CH}_{2} \mathrm{~S}\right), 20.8\left(\mathrm{CH}_{2} \mathrm{CH}_{3}\right), 28.7\left(\mathrm{CH}_{2} \mathrm{CS}\right)$, $31.5\left(\mathrm{CH}_{2} \mathrm{CF}_{2}, \mathrm{t}, \mathrm{J}=22.7 \mathrm{~Hz}\right), 42.2\left(\mathrm{CH}_{2} \mathrm{~N}\right), 49.3\left(\mathrm{CH}_{2} \mathrm{SO}_{2}\right), 52.5(C \mathrm{Ar}), 110.7(\mathrm{ArCH})$, 
$116.3(\mathrm{ArC}), 127.4(\mathrm{ArCH}), 129.8(\mathrm{ArC}), 131.9\left(\mathrm{CH}_{2}=\mathrm{CH}\right), 133.1(\mathrm{ArCH}), 135.4$ $\left(\mathrm{CH}=\mathrm{CH}_{2}\right), 141.4(\mathrm{ArC}), 174.7(\mathrm{C}=\mathrm{O})$.

IR $v_{\max } /\left(\mathrm{cm}^{-1}\right): 3421,2093,1702,1608,1482,1204$.

MS $m / z$ (ES ${ }^{+}$mode): $869\left(\mathrm{M}^{+}+\mathrm{H}+\mathrm{NH}_{4}, 37 \%\right), 789$ (18), 611 (12), 581 (19), 490 (58), 391 (70), 367 (81), 265 (22), 225 (11), 191 (22), 153 (100).

$\mathrm{C}_{25} \mathrm{H}_{25} \mathrm{O}_{3} \mathrm{~N}_{2} \mathrm{BrF}_{17} \mathrm{~S}_{2}$ requires 867.0213, found 867.0192.

\section{3-(2-Vinylsulfonylethyl)-3-(3,3,4,4,5,5,6,6,7,7,8,8,9,9,10,10,10-}

heptadecafluorodecylsulfanyl)-1-methyl-1,3-dihydroindol-2-one (6)

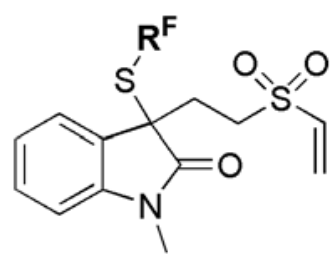

To a solution of 3-(3,3,4,4,5,5,6,6,7,7,8,8,9,9,10,10,10-heptadecafluorodecylsulfanyl)-1methyl-1,3-dihydroindol-2-one (0.075 g, $0.120 \mathrm{mmol}, 1 \mathrm{eq})$ in $\mathrm{CH}_{2} \mathrm{Cl}_{2}(5 \mathrm{ml})$ was added DBU $(0.050 \mathrm{ml}, 0.300 \mathrm{mmol}, 2.5 \mathrm{eq})$ and divinyl sulfone $(0.020 \mathrm{ml}, 0.156 \mathrm{mmol}, 1.3 \mathrm{eq})$ at room temperature and the reaction allowed to stir for 18 hours. The reaction mixture was then quenched with aqueous saturated $\mathrm{NH}_{4} \mathrm{Cl}(2 \mathrm{ml})$ and the organic layer dried $\left(\mathrm{MgSO}_{4}\right)$, filtered and concentrated in vacuo to give the crude product as a yellow solid which was purified by fluorous chromatography to give 3-(2-vinylsulfonylethyl)-3(3,3,4,4,5,5,6,6,7,7,8,8,9,9,10,10,10-heptadecafluorodecylsulfanyl)-1-methyl-1,3dihydroindol-2-one $6(0.083 \mathrm{~g}, 0.112 \mathrm{mmol}, 93 \%)$ as a yellow solid (mpt $71.0-72.0{ }^{\circ} \mathrm{C}$ recrystallized from $\mathrm{MeOH})$.

${ }^{1} \mathrm{H}$ NMR (500 MHz, $\mathrm{CDCl}_{3}$ ) $\delta$ 2.10-2.21 (2H, m, $\left.\mathrm{CH}_{2} \mathrm{CF}_{2}\right), 2.42-2.53$ (2H, m, CH 2.57-2.63 $\left(1 \mathrm{H}, \mathrm{m}, 1 \mathrm{H}\right.$ of $\left.\mathrm{CH}_{2} \mathrm{~S}\right), 2.66-2.72\left(1 \mathrm{H}, \mathrm{m}, 1 \mathrm{H}\right.$ of $\left.\mathrm{CH}_{2} \mathrm{~S}\right), 2.88-2.91(2 \mathrm{H}, \mathrm{m}$, $\left.\mathrm{CH}_{2} \mathrm{SO}_{2}\right), 3.24\left(3 \mathrm{H}, \mathrm{s}, \mathrm{CH}_{3}\right), 6.21\left(1 \mathrm{H}, \mathrm{d}, \mathrm{J}=9.6 \mathrm{~Hz}, 1 \mathrm{H}\right.$ of $\left.\mathrm{CH}_{2}=\mathrm{CH}\right), 6.45(1 \mathrm{H}, \mathrm{d}, \mathrm{J}=$ $16.5 \mathrm{~Hz}, 1 \mathrm{H}$ of $\left.\mathrm{CH}_{2}=\mathrm{CH}\right), 6.57\left(1 \mathrm{H}, \mathrm{dd}, \mathrm{J}=9.6,16.5 \mathrm{~Hz}, \mathrm{CH}=\mathrm{CH}_{2}\right), 6.90(1 \mathrm{H}, \mathrm{d}, \mathrm{J}=7.6$ 
$\mathrm{Hz}, \operatorname{ArCH}), 7.17(1 \mathrm{H}, \mathrm{dt}, \mathrm{J}=1.1,7.6 \mathrm{~Hz}, \operatorname{ArCH}), 7.32(1 \mathrm{H}, \mathrm{d}, \mathrm{J}=7.6 \mathrm{~Hz}, \operatorname{ArCH}), 7.39$ $(1 \mathrm{H}, \mathrm{dt}, \mathrm{J}=1.1,7.6 \mathrm{~Hz}, \mathrm{ArCH})$.

${ }^{13} \mathrm{C}$ NMR (126 MHz, $\left.\mathrm{CDCl}_{3}\right)$ d $19.9\left(\mathrm{CH}_{2} \mathrm{~S}\right), 26.7\left(\mathrm{CH}_{3}\right), 28.8\left(\mathrm{CH}_{2} \mathrm{CS}\right), 31.5\left(\mathrm{CH}_{2} \mathrm{CF}_{2}, \mathrm{t}\right.$, $\mathrm{J}=22.7 \mathrm{~Hz}), 49.5\left(\mathrm{CH}_{2} \mathrm{SO}_{2}\right), 52.8(\mathrm{CAr}), 109.0(\mathrm{ArCH}), 124.0(\mathrm{ArCH}), 124.1(\mathrm{ArCH})$, $127.5(\mathrm{ArC}), 130.3(\mathrm{ArCH}), 131.8\left(\mathrm{CH}_{2}=\mathrm{CH}\right), 135.5\left(\mathrm{CH}=\mathrm{CH}_{2}\right), 143.0(\mathrm{ArC}), 175.4$ $(C=\mathrm{O})$.

IR $v_{\max } /\left(\mathrm{cm}^{-1}\right): 3418,2306,1712,1612,1422,1319,1270,1152,982,896$.

MS $m / z$ (ES ${ }^{+}$mode): $766\left(\mathrm{M}^{+}+\mathrm{Na}, 100 \%\right), 389$ (23).

$\mathrm{C}_{23} \mathrm{H}_{18} \mathrm{O}_{3} \mathrm{NF}_{17} \mathrm{NaS}_{2}$ requires 766.0349 , found 766.0337 .

\section{Spirocyclic sulfone (7)}

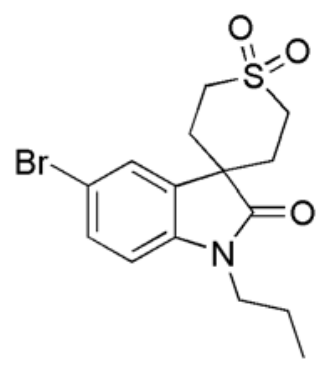

To a solution of 5-bromo-3-(2-vinylsulfonylethyl)-3-(3,3,4,4,5,5,6,6,7,7,8,8,9,9,10,10,10heptadecafluorodecylsulfonyl)-1-propyl-1,3-dihydroindol-2-one (0.229 g, $0.269 \mathrm{mmol}, 1$ eq) in degassed THF $(24 \mathrm{ml})$ was added $\mathrm{SmI}_{2}(6 \mathrm{ml}, 0.1 \mathrm{M}$ solution in THF, $0.592 \mathrm{mmol}$, 2.2 eq) at room temperature and the reaction allowed to stir for 7 hours. The reaction mixture was then stirred in the presence of air until it turned yellow in colour. The reaction was then quenched with aqueous saturated $\mathrm{NaHCO}_{3}(10 \mathrm{ml})$ and the aqueous layer extracted with EtOAc $(3 \times 10 \mathrm{ml})$. The organic layer was dried $\left(\mathrm{MgSO}_{4}\right)$, filtered and concentrated in vacuo to give the crude product as a yellow oil which was purified by flash chromatography using $10 \%$ ethyl acetate in petroleum ether to give $7(0.062 \mathrm{~g}$, $0.167 \mathrm{mmol}, 62 \%$ ) as a white solid ( $\mathrm{mpt} 184.8-186.3{ }^{\circ} \mathrm{C}$ recrystallized from $\mathrm{CHCl}_{3}$ ).

${ }^{1} \mathrm{H}$ NMR (500 MHz, $\left.\mathrm{CDCl}_{3}\right) \delta 0.95\left(3 \mathrm{H}, \mathrm{t}, \mathrm{J}=7.2 \mathrm{~Hz}, \mathrm{CH}_{3}\right), 1.66-1.74\left(2 \mathrm{H}, \mathrm{m}, \mathrm{CH}_{2} \mathrm{CH}_{3}\right)$, $2.06\left(2 \mathrm{H}, \mathrm{d}, \mathrm{J}=15.0 \mathrm{~Hz}, \mathrm{CH}_{2}\right), 2.65\left(2 \mathrm{H}, \mathrm{dt}, \mathrm{J}=3.5,15.0 \mathrm{~Hz}, \mathrm{CH}_{2}\right), 2.95(2 \mathrm{H}, \mathrm{dd}, \mathrm{J}=3.5$, 
$\left.15.0 \mathrm{~Hz}, \mathrm{CH}_{2}\right), 3.66\left(2 \mathrm{H}, \mathrm{t}, \mathrm{J}=7.2 \mathrm{~Hz}, \mathrm{CH}_{2} \mathrm{~N}\right), 4.05\left(2 \mathrm{H}, \mathrm{dt}, \mathrm{J}=3.5,13.9 \mathrm{~Hz}, \mathrm{CH}_{2}\right), 6.77$ $(1 \mathrm{H}, \mathrm{d}, \mathrm{J}=8.2 \mathrm{~Hz}, \operatorname{ArCH}), 7.39(1 \mathrm{H}, \mathrm{d}, \mathrm{J}=2.1 \mathrm{~Hz}, \operatorname{ArCH}), 7.44(1 \mathrm{H}, \mathrm{dd}, \mathrm{J}=2.1,8.2 \mathrm{~Hz}$, $\mathrm{ArCH})$.

${ }^{13} \mathrm{C}$ NMR $\left(126 \mathrm{MHz}, \mathrm{CDCl}_{3}\right) \delta 11.4\left(\mathrm{CH}_{3}\right), 20.7\left(\mathrm{CH}_{2} \mathrm{CH}_{3}\right), 32.0\left(2 \times \mathrm{CH}_{2}\right), 41.5$ $\left(\mathrm{CH}_{2} \mathrm{~N}\right), 43.6(\mathrm{CAr}), 46.1\left(2 \times \mathrm{CH}_{2}\right), 110.5(\mathrm{ArCH}), 115.7(\mathrm{ArC}), 126.2(\mathrm{ArCH}), 131.8$ $(\operatorname{ArCH}), 134.0(\operatorname{ArC}), 141.0(\operatorname{ArC}), 177.5(C=\mathrm{O})$.

IR $v_{\max } /\left(\mathrm{cm}^{-1}\right): 3436,2305,2125,1702,1608,1422,1269,1126,896$.

MS $m / z$ (CI mode): $389\left(\mathrm{M}^{+}+\mathrm{NH}_{4}, 12 \%\right), 374$ (19), 311 (40), 294 (31), 160 (10), 138 (10), 127 (22), 113 (40), 96 (35), 90 (80), 84 (37), 71 (40), 58 (100).

$\mathrm{C}_{15} \mathrm{H}_{19} \mathrm{O}_{3} \mathrm{NBrS}$ requires 372.0264 , found 372.0259 .

\section{Spirocyclic sulfone (8)}

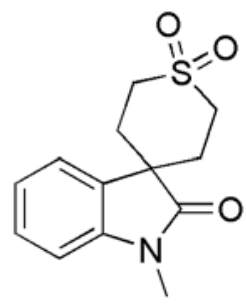

To a solution of 3-(2-vinylsulfonylethyl)-3-(3,3,4,4,5,5,6,6,7,7,8,8,9,9,10,10,10heptadecafluorodecylsulfanyl)-1-methyl-1,3-dihydroindol-2-one (0.167 g, $0.225 \mathrm{mmol}, 1$ eq) in degassed THF $(10 \mathrm{ml})$ was added $\mathrm{SmI}_{2}(5 \mathrm{ml}, 0.1 \mathrm{M}$ solution in THF, $0.494 \mathrm{mmol}$, 2.2 eq) at room temperature and the reaction allowed to stir for 3 hours. The reaction mixture was then stirred in the presence of air until it turned yellow in colour. The reaction was then quenched with aqueous saturated $\mathrm{NaHCO}_{3}(5 \mathrm{ml})$ and the aqueous layer extracted with EtOAc $(3 \times 5 \mathrm{ml})$. The organic layer was dried $\left(\mathrm{MgSO}_{4}\right)$, filtered and concentrated in vacuo to give the crude product as a yellow solid which was purified by flash chromatography using $20 \%$ ethyl acetate in petroleum ether to give $8(0.239 \mathrm{~g}$, $0.090 \mathrm{mmol}, 40 \%$ ) as a white solid (mpt $257.2-259.6{ }^{\circ} \mathrm{C}$ recrystallized from $\mathrm{CHCl}_{3}$ ). 
${ }^{1} \mathrm{H}$ NMR $\left(500 \mathrm{MHz}, \mathrm{CDCl}_{3}\right) \delta 2.08\left(2 \mathrm{H}, \mathrm{d}, \mathrm{J}=15.2 \mathrm{~Hz}, \mathrm{CH} \mathrm{CAr}_{2}\right), 2.68(2 \mathrm{H}, \mathrm{dt}, \mathrm{J}=3.6$, $\left.15.2 \mathrm{~Hz}, \mathrm{CH}_{2} \mathrm{CAr}\right), 2.95\left(2 \mathrm{H}, \mathrm{dd}, \mathrm{J}=3.6,14.3 \mathrm{~Hz}, \mathrm{CH}_{2} \mathrm{SO}_{2}\right), 3.23\left(3 \mathrm{H}, \mathrm{s}, \mathrm{CH}_{3}\right), 4.07(2 \mathrm{H}$, $\left.\mathrm{dt}, \mathrm{J}=3.6,14.3 \mathrm{~Hz}, \mathrm{CH}_{2} \mathrm{SO}_{2}\right), 6.89(1 \mathrm{H}, \mathrm{d}, \mathrm{J}=7.9 \mathrm{~Hz}, \mathrm{ArCH}), 7.13(1 \mathrm{H}, \mathrm{dt}, \mathrm{J}=1.3,7.9$ $\mathrm{Hz}, \mathrm{ArCH}), 7.28(1 \mathrm{H}, \mathrm{d}, \mathrm{J}=7.4 \mathrm{~Hz}, \mathrm{ArCH}), 7.34(1 \mathrm{H}, \mathrm{dt}, \mathrm{J}=1.3,7.4 \mathrm{~Hz}, \mathrm{ArCH})$.

${ }^{13} \mathrm{C}$ NMR $\left(126 \mathrm{MHz}, \mathrm{CDCl}_{3}\right) \delta 26.3\left(\mathrm{CH}_{3}\right), 32.0\left(2 \times \mathrm{CH}_{2} \mathrm{CAr}\right), 43.6(\mathrm{CAr}), 46.3(2 \times$ $\left.\mathrm{CH}_{2} \mathrm{SO}_{2}\right), 108.7(\mathrm{ArCH}), 122.6(\mathrm{ArCH}), 123.4(\mathrm{ArCH}), 129.0(\mathrm{ArCH}), 131.9(\mathrm{ArC})$, $142.5(\mathrm{ArC}), 178.0(C=\mathrm{O})$.

IR $v_{\max } /\left(\mathrm{cm}^{-1}\right): 3429,2305,1700,1613,1472,1265,1128,860,738,425$.

MS $m / z\left(\mathrm{ES}^{+}\right.$mode): $288\left(\mathrm{M}^{+}+\mathrm{Na}, 100 \%\right), 234$ (3), 179 (1).

$\mathrm{C}_{13} \mathrm{H}_{16} \mathrm{O}_{3} \mathrm{NS}$ requires 266.0845, found 266.0848.

\section{5-Bromo-3-(2-vinylsulfonylethyl)-1-propyl-1,3-dihydroindol-2-one (9)}

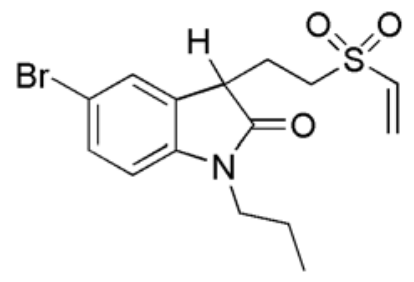

To a degassed solution of 5-bromo-3-(2-vinylsulfonylethyl)-3(3,3,4,4,5,5,6,6,7,7,8,8,9,9,10,10,10-heptadecafluorodecylsulfonyl)-1-propyl-1,3-

dihydroindol-2-one (0.235 g, $0.277 \mathrm{mmol}, 1 \mathrm{eq})$ and $\mathrm{MeOH}(0.010 \mathrm{ml}, 0.277 \mathrm{mmol}, 1 \mathrm{eq})$ in THF (25 ml) was added $\mathrm{SmI}_{2}(6 \mathrm{ml}, 0.1 \mathrm{M}$ solution in THF, $0.608 \mathrm{mmol}, 2.2 \mathrm{eq})$ at room temperature and the reaction allowed to stir for 20 mins. The reaction mixture was then quenched with aqueous saturated $\mathrm{NaHCO}_{3}(7 \mathrm{ml})$ and the aqueous layer extracted with EtOAc $(3 \times 10 \mathrm{ml})$. The organic layer was then dried $\left(\mathrm{MgSO}_{4}\right)$, filtered and concentrated in vacuo to give the crude product as a white oil which was purified by flash chromatography using $10 \%$ ethyl acetate in petroleum ether to give 5-bromo-3-(2vinylsulfonylethyl)-1-propyl-1,3-dihydroindol-2-one 9 (0.074 g, $0.199 \mathrm{mmol}, 72 \%)$ as a yellow oil. 
${ }^{1} \mathrm{H}$ NMR (300 MHz, $\left.\mathrm{CDCl}_{3}\right) \delta 0.93\left(3 \mathrm{H}, \mathrm{t}, \mathrm{J}=7.4 \mathrm{~Hz}, \mathrm{CH}_{3}\right), 1.61-1.72\left(2 \mathrm{H}, \mathrm{m}, \mathrm{CH}_{2} \mathrm{CH}_{3}\right)$, 2.18-2.27 $\left(1 \mathrm{H}, \mathrm{m}, 1 \mathrm{H}\right.$ of $\left.\mathrm{CH}_{2} \mathrm{CH}\right), 2.40-2.52\left(1 \mathrm{H}, \mathrm{m}, 1 \mathrm{H}\right.$ of $\left.\mathrm{CH}_{2} \mathrm{CH}\right), 3.07-3.25(2 \mathrm{H}, \mathrm{m}$, $\left.\mathrm{CH}_{2} \mathrm{SO}_{2}\right), 3.56(1 \mathrm{H}, \mathrm{dd}, \mathrm{J}=2.9,8.1 \mathrm{~Hz}, \mathrm{CH}), 3.62\left(2 \mathrm{H}, \mathrm{t}, \mathrm{J}=7.4 \mathrm{~Hz}, \mathrm{CH}_{2} \mathrm{~N}\right), 6.20(1 \mathrm{H}, \mathrm{d}$, $\mathrm{J}=9.8 \mathrm{~Hz}, 1 \mathrm{H}$ of $\left.\mathrm{CH}_{2}=\mathrm{CH}\right), 6.44\left(1 \mathrm{H}, \mathrm{d}, \mathrm{J}=16.6 \mathrm{~Hz}, 1 \mathrm{H}\right.$ of $\left.\mathrm{CH}_{2}=\mathrm{CH}\right), 6.64(1 \mathrm{H}, \mathrm{dd}, \mathrm{J}=$ 9.8, 16.6 Hz, $\left.\mathrm{CH}=\mathrm{CH}_{2}\right), 6.73(1 \mathrm{H}, \mathrm{d}, \mathrm{J}=8.3 \mathrm{~Hz}, \mathrm{ArCH}), 7.40(2 \mathrm{H}$, apparent td, J = 8.3, $2.0 \mathrm{~Hz}, 2 \times \mathrm{ArCH})$.

${ }^{13} \mathrm{C}$ NMR $\left(75 \mathrm{MHz}, \mathrm{CDCl}_{3}\right) \delta 11.5\left(\mathrm{CH}_{3}\right), 20.8\left(\mathrm{CH}_{2} \mathrm{CH}_{3}\right), 23.6\left(\mathrm{CH}_{2} \mathrm{CH}\right), 41.9\left(\mathrm{CH}_{2} \mathrm{~N}\right)$, $43.4(\mathrm{CH}), 50.4\left(\mathrm{CH}_{2} \mathrm{SO}_{2}\right), 110.2(\mathrm{ArCH}), 115.4(\mathrm{ArC}), 127.3(\mathrm{ArCH}), 129.4(\mathrm{ArC})$, $131.4\left(\mathrm{CH}_{2}=\mathrm{CH}\right), 131.6(\mathrm{ArCH}), 135.9\left(\mathrm{CH}=\mathrm{CH}_{2}\right), 142.8(\mathrm{ArC}), 175.9(C=\mathrm{O})$.

IR $v_{\max } /\left(\mathrm{cm}^{-1}\right): 3054,2305,1708,1608,1484,1421,1251,1128,896$.

MS m/z (ES mode): $394\left(\mathrm{M}^{+}+\mathrm{Na}, 84\right.$ \%), 374 (18), 316 (13), 237 (5), 161 (6).

$\mathrm{C}_{15} \mathrm{H}_{18} \mathrm{O}_{3} \mathrm{NBrNaS}$ requires 394.0083, found 394.0092.

\section{5-Bromo-3-\{2-[2-(3,3,4,4,5,5,6,6,7,7,8,8,9,9,10,10,10-heptadecafluorodecylsulfanyl)-} ethanesulfonyl]-ethyl\}-1-propyl-1,3-dihydroindol-2-one (10)

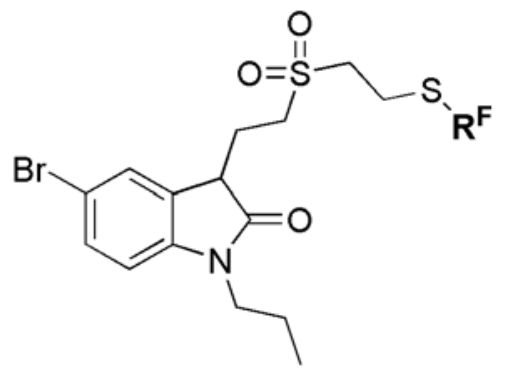

To a degassed solution of 5-bromo-3-(2-vinylsulfonylethyl)-3$(3,3,4,4,5,5,6,6,7,7,8,8,9,9,10,10,10$-heptadecafluorodecylsulfonyl)-1-propyl-1,3dihydroindol-2-one $(0.277 \mathrm{~g}, 0.326 \mathrm{mmol}, 1 \mathrm{eq})$ in THF $(7 \mathrm{ml})$ was added $\mathrm{SmI}_{2}(7.2 \mathrm{ml}$, $0.1 \mathrm{M}$ solution in THF, $0.716 \mathrm{mmol}, 2.2 \mathrm{eq}$ ) at room temperature and the reaction allowed to stir for 3 hours. Once yellow in colour the reaction mixture was quenched with aqueous saturated $\mathrm{NaHCO}_{3}(3 \mathrm{ml})$ and the aqueous layer extracted with EtOAc $(3 \times 5 \mathrm{ml})$. The organic layer was then dried $\left(\mathrm{MgSO}_{4}\right)$, filtered and concentrated in vacuo to give the crude product as a white solid which was purified by flash chromatography using $10 \%$ ethyl acetate in petroleum ether to give $7(0.048 \mathrm{~g}, 0.129 \mathrm{mmol}, 40 \%)$ as a white solid 
and 5-bromo-3-\{2-[2-(3,3,4,4,5,5,6,6,7,7,8,8,9,9,10,10,10-heptadecafluorodecylsulfanyl)ethanesulfonyl]-ethyl\}-1-propyl-1,3-dihydroindol-2-one 10 (0.034 g, $0.040 \mathrm{mmol}, 12 \%)$ as a white solid (mpt $114.8-115.7^{\circ} \mathrm{C}$ recrystallized from $\left.\mathrm{EtOH}\right)$.

${ }^{1} \mathrm{H}$ NMR $\left(300 \mathrm{MHz}, \mathrm{CDCl}_{3}\right) \delta 0.95\left(3 \mathrm{H}, \mathrm{t}, \mathrm{J}=7.4 \mathrm{~Hz}, \mathrm{CH}_{3}\right), 1.62-1.75\left(2 \mathrm{H}, \mathrm{m}, \mathrm{CH}_{2} \mathrm{CH}_{3}\right)$, 2.19-2.30 $\left(1 \mathrm{H}, \mathrm{m}, 1 \mathrm{H}\right.$ of $\left.\mathrm{CH}_{2}\right), 2.32-2.52\left(2 \mathrm{H}, \mathrm{m}, \mathrm{CH}_{2} \mathrm{CF}_{2}\right), 2.54-2.64(1 \mathrm{H}, \mathrm{m}, 1 \mathrm{H}$ of $\left.\mathrm{CH}_{2}\right), 2.79-2.85\left(2 \mathrm{H}, \mathrm{m}, \mathrm{CH}_{2}\right), 2.96-3.04\left(2 \mathrm{H}, \mathrm{m}, \mathrm{CH}_{2}\right), 3.24-3.30\left(4 \mathrm{H}, \mathrm{m}, 2 \times \mathrm{CH}_{2}\right)$, 3.59-3.67 $\left(3 \mathrm{H}, \mathrm{m}, \mathrm{CH}\right.$ and $\left.\mathrm{CH}_{2} \mathrm{~N}\right), 6.75(1 \mathrm{H}, \mathrm{d}, \mathrm{J}=8.2 \mathrm{~Hz}, \mathrm{ArCH}), 7.40-7.45(2 \mathrm{H}, \mathrm{m}, 2 \times$ $\mathrm{ArCH})$.

${ }^{13} \mathrm{C}$ NMR $\left(75 \mathrm{MHz}, \mathrm{CDCl}_{3}\right) \delta 11.6\left(\mathrm{CH}_{3}\right), 20.9\left(\mathrm{CH}_{2} \mathrm{CH}_{3}\right), 23.3\left(\mathrm{CH}_{2}\right), 23.5\left(\mathrm{CH}_{2}\right), 24.4$ $\left(\mathrm{CH}_{2}\right), 32.0\left(\mathrm{CH}_{2} \mathrm{CF}_{2}, \mathrm{t}, \mathrm{J}=22.0 \mathrm{~Hz}\right), 42.0\left(\mathrm{CH}_{2} \mathrm{~N}\right), 43.4(\mathrm{CH}), 49.9\left(\mathrm{CH}_{2}\right), 53.1\left(\mathrm{CH}_{2}\right)$, $110.3(\mathrm{ArCH}), 115.6(\mathrm{ArC}), 127.5(\mathrm{ArCH}), 129.4(\mathrm{ArC}), 131.8(\mathrm{ArCH}), 142.9(\mathrm{ArC})$, $176.1(C=\mathrm{O})$.

IR $v_{\max } /\left(\mathrm{cm}^{-1}\right): 3418,3056,2306,1709,1609,1484,1428,1268,1146,896,811$.

MS $m / z$ (ES ${ }^{+}$mode): $875\left(\mathrm{M}^{+}+\mathrm{H}+\mathrm{Na}, 100 \%\right.$ ), 685 (34), 663 (23), 644 (6), 560 (7), 482 (11), 463 (10), 302 (6).

$\mathrm{C}_{25} \mathrm{H}_{23} \mathrm{O}_{3} \mathrm{NBrF}_{17} \mathrm{NaS}_{2}$ requires 873.9923, found 873.9949.

\section{5-Bromo-3-(3,3,4,4,5,5,6,6,7,7,8,8,9,9,10,10,10-heptadecafluoro-decane-1-sulfonyl)-3-} (2-nitrobenzyl)-1-propyl-1,3-dihydroindol-2-one (12)

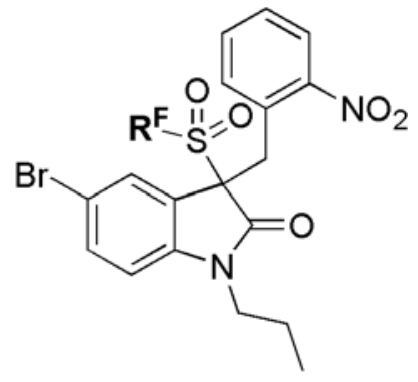

To a solution of 5-bromo-3-(3,3,4,4,5,5,6,6,7,7,8,8,9,9,10,10,10-heptadecafluorodecane1-sulfonyl)-1-propyl-1,3-dihydroindol-2-one (0.199 g, $0.260 \mathrm{mmol}, 1 \mathrm{eq})$ in DMF (7.0 $\mathrm{ml})$ was added $\mathrm{K}_{2} \mathrm{CO}_{3}(0.180 \mathrm{~g}, 1.30 \mathrm{mmol}, 5 \mathrm{eq})$ and 2-nitrobenzyl bromide $(0.281 \mathrm{~g}$, 
$1.30 \mathrm{mmol}, 5 \mathrm{eq})$ at room temperature and the reaction allowed to stir for 19 hours. The reaction mixture was then diluted with EtOAc $(10 \mathrm{ml})$ and washed with water $(5 \times 15$ $\mathrm{ml})$. The organic layer was then dried $\left(\mathrm{MgSO}_{4}\right)$, filtered and concentrated in vacuo to give the crude product as a yellow oil which was purified by fluorous chromatography to give 5-bromo-3-(3,3,4,4,5,5,6,6,7,7,8,8,9,9,10,10,10-heptadecafluorodecane-1-sulfonyl)3-(2-nitrobenzyl)-1-propyl-1,3-dihydroindol-2-one 12 (0.214 g, $0.238 \mathrm{mmol}, 92 \%)$ as a yellow oil.

${ }^{1} \mathrm{H}$ NMR $\left(300 \mathrm{MHz}, \mathrm{CDCl}_{3}\right) \delta 0.79\left(3 \mathrm{H}, \mathrm{t}, \mathrm{J}=7.4 \mathrm{~Hz}, \mathrm{CH}_{3}\right), 1.42-1.54\left(2 \mathrm{H}, \mathrm{m}, \mathrm{CH}_{2} \mathrm{CH}_{3}\right)$, 2.63-2.82 $\left(2 \mathrm{H}, \mathrm{m}, \mathrm{CH}_{2} \mathrm{CF}_{2}\right), 3.44-3.53\left(1 \mathrm{H}, \mathrm{m}, 1 \mathrm{H}\right.$ of $\left.\mathrm{CH}_{2} \mathrm{SO}_{2}\right), 3.55-3.66(2 \mathrm{H}, \mathrm{m}$, $\left.\mathrm{CH}_{2} \mathrm{~N}\right), 3.85-3.96\left(1 \mathrm{H}, \mathrm{m}, 1 \mathrm{H}\right.$ of $\left.\mathrm{CH}_{2} \mathrm{SO}_{2}\right), 4.16\left(1 \mathrm{H}, \mathrm{d}, \mathrm{J}=13.8 \mathrm{~Hz}, 1 \mathrm{H}\right.$ of $\left.\mathrm{CH}_{2} \mathrm{Ph}\right), 4.46$ $\left(1 \mathrm{H}, \mathrm{d}, \mathrm{J}=13.8 \mathrm{~Hz}, 1 \mathrm{H}\right.$ of $\left.\mathrm{CH}_{2} \mathrm{Ph}\right), 6.65(1 \mathrm{H}, \mathrm{d}, \mathrm{J}=8.5 \mathrm{~Hz}, \mathrm{ArCH}), 7.33(1 \mathrm{H}, \mathrm{td}, \mathrm{J}=8.0$, $1.4 \mathrm{~Hz}, \operatorname{ArCH}), 7.38(2 \mathrm{H}, \mathrm{dd}, \mathrm{J}=1.7,8.0 \mathrm{~Hz}, 2 \times \operatorname{ArCH}), 7.46(1 \mathrm{H}, \mathrm{td}, \mathrm{J}=8.0,1.4 \mathrm{~Hz}$, $\operatorname{ArCH}), 7.59(1 \mathrm{H}, \mathrm{d}, \mathrm{J}=2.1 \mathrm{~Hz}, \operatorname{ArCH}), 7.78(1 \mathrm{H}, \mathrm{dd}, \mathrm{J}=1.4,8.0 \mathrm{~Hz}, \mathrm{ArCH})$.

${ }^{13} \mathrm{C}$ NMR $\left(75 \mathrm{MHz}, \mathrm{CDCl}_{3}\right) \delta 11.1\left(\mathrm{CH}_{3} \mathrm{CH}_{2}\right), 20.5\left(\mathrm{CH}_{2} \mathrm{CH}_{3}\right), 24.1\left(\mathrm{CH}_{2} \mathrm{CF}_{2}, \mathrm{t}, \mathrm{J}=22.6\right.$ $\mathrm{Hz}), 33.1\left(\mathrm{CH}_{2} \mathrm{Ph}\right), 40.9\left(\mathrm{CH}_{2} \mathrm{~N}\right), 42.5\left(\mathrm{CH}_{2} \mathrm{SO}_{2}\right), 74.5\left(\mathrm{CSO}_{2}\right), 110.6(\mathrm{ArCH}), 116.4$ $(\mathrm{ArC}), 121.6(\mathrm{ArC}), 125.5(\mathrm{ArCH}), 127.5(\mathrm{ArC}), 129.2(\mathrm{ArCH}), 130.1(\mathrm{ArCH}), 133.0$ $(\mathrm{ArCH}), 133.3(\mathrm{ArCH}), 134.3(\mathrm{ArCH}), 143.4(\mathrm{ArC}), 149.8(\mathrm{ArC}), 169.6(C=\mathrm{O})$.

IR $v_{\max } /\left(\mathrm{cm}^{-1}\right): 3421,1715,1636,1265,738$.

MS $m / z$ ( $\left(\mathrm{SS}^{+}\right.$mode): $916\left(\mathrm{M}^{+}+\mathrm{NH}_{4}, 87\right.$ \%), 843 (31), 838 (48), 739 (16), 654 (9), 537

(18), 288 (100), 242 (13), 160 (18), 145 (79).

$\mathrm{C}_{28} \mathrm{H}_{24} \mathrm{O}_{5} \mathrm{~N}_{3} \mathrm{BrF}_{17} \mathrm{~S}$ requires 916.0343 , found 916.0333 .

3-(2-Aminobenzyl)-5-bromo-1-propyl-1,3-dihydroindol-2-one (13)<smiles>CCCN1C(=O)C(Cc2ccccc2N)c2cc(Br)ccc21</smiles> 
To a degassed solution of 5-bromo-3-(3,3,4,4,5,5,6,6,7,7,8,8,9,9,10,10,10heptadecafluorodecane-1-sulfonyl)-3-(2-nitrobenzyl)-1-propyl-1,3-dihydroindol-2-one $(0.301 \mathrm{~g}, 0.335 \mathrm{mmol}, 1 \mathrm{eq})$ in THF $(12 \mathrm{ml})$ was added $\mathrm{SmI}_{2}(47 \mathrm{ml}, 0.1 \mathrm{M}$ solution in THF, $4.69 \mathrm{mmol}, 14 \mathrm{eq}$ ) at room temperature and the reaction allowed to stir for 48 hours. The reaction was then quenched with aqueous saturated $\mathrm{NaHCO}_{3}(20 \mathrm{ml})$ and the aqueous layer extracted with EtOAc $(3 \times 10 \mathrm{ml})$. The organic layer was then dried $\left(\mathrm{MgSO}_{4}\right)$, filtered and concentrated in vacuo to give the crude product as a yellow oil which was purified by flash chromatography using $10 \%$ ethyl acetate in petroleum ether to give 3-(2-aminobenzyl)-5-bromo-1-propyl-1,3-dihydroindol-2-one 13 (0.048 g, 0.134 mmol, $40 \%)$ as a yellow oil.

${ }^{1} \mathrm{H}$ NMR $\left(300 \mathrm{MHz}, \mathrm{CDCl}_{3}\right) \delta 0.89\left(3 \mathrm{H}, \mathrm{t}, \mathrm{J}=7.4 \mathrm{~Hz}, \mathrm{CH}_{3}\right), 1.58-1.71\left(2 \mathrm{H}, \mathrm{m}, \mathrm{CH}_{2} \mathrm{CH}_{3}\right)$, $2.93\left(1 \mathrm{H}, \mathrm{dd}, \mathrm{J}=8.9,14.2 \mathrm{~Hz}, 1 \mathrm{H}\right.$ of $\left.\mathrm{CH}_{2} \mathrm{Ar}\right), 3.34(1 \mathrm{H}, \mathrm{dd}, \mathrm{J}=3.8,14.2 \mathrm{~Hz}, 1 \mathrm{H}$ of $\left.\mathrm{CH}_{2} \mathrm{Ar}\right), 3.56-3.65\left(1 \mathrm{H}, \mathrm{m}, 1 \mathrm{H}\right.$ of $\left.\mathrm{CH}_{2} \mathrm{~N}\right), 3.67-3.77\left(1 \mathrm{H}, \mathrm{m}, 1 \mathrm{H}\right.$ of $\left.\mathrm{CH}_{2} \mathrm{~N}\right), 3.85(1 \mathrm{H}, \mathrm{dd}$, $\mathrm{J}=3.8,8.9 \mathrm{~Hz}, \mathrm{CH}), 4.03\left(2 \mathrm{H}\right.$, broad s, $\left.\mathrm{NH}_{2}\right), 6.73(3 \mathrm{H}, \mathrm{t}, \mathrm{J}=6.8 \mathrm{~Hz}, 3 \times \operatorname{ArCH}), 6.91$ $(1 \mathrm{H}, \mathrm{dd}, \mathrm{J}=1.1,7.4 \mathrm{~Hz}, \operatorname{ArCH}), 7.07-7.14(2 \mathrm{H}, \mathrm{m}, 2 \times \mathrm{ArCH}), 7.41(1 \mathrm{H}, \mathrm{ddd}, \mathrm{J}=1.1$, 2.0, $8.3 \mathrm{~Hz}, \mathrm{ArCH})$.

${ }^{13} \mathrm{C}$ NMR $\left(75 \mathrm{MHz}, \mathrm{CDCl}_{3}\right) \delta 11.4\left(\mathrm{CH}_{3}\right), 20.8\left(\mathrm{CH}_{2} \mathrm{CH}_{3}\right), 32.9\left(\mathrm{CH}_{2} \mathrm{Ar}\right), 41.8\left(\mathrm{CH}_{2} \mathrm{~N}\right)$, $45.0(\mathrm{CH}), 109.9(\mathrm{ArCH}), 114.9(\mathrm{ArC}), 116.4(\mathrm{ArCH}), 118.6(\mathrm{ArCH}), 122.4(\mathrm{ArC}), 128.1$ ( $\mathrm{ArCH}), 128.3(\mathrm{ArCH}), 130.8(\mathrm{ArCH}), 131.3(\mathrm{ArC}), 131.3(\mathrm{ArCH}), 142.9(\mathrm{ArC}), 145.0$ $(\operatorname{ArC}), 177.6(C=\mathrm{O})$.

IR $v_{\max } /\left(\mathrm{cm}^{-1}\right): 3436,2306,2126,1639,1421,1261,896$.

MS $m / z$ (ES mode): 359 (M+ H, 31 \%), 344 (10), 303 (100), 281 (46), 212 (9), 145 (6), 106 (47), 90 (25).

$\mathrm{C}_{18} \mathrm{H}_{20} \mathrm{ON}_{2} \mathrm{Br}$ requires 359.0754 , found 359.0749 .

\section{9-Bromo-6-propyl-6H-indolo[2,3-b]quinoline (14)}




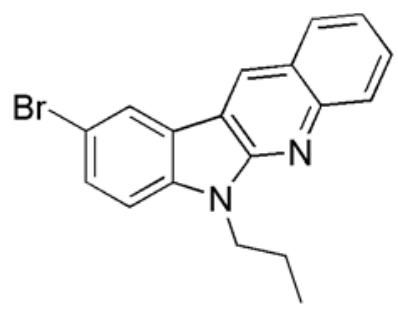

A solution of 3-(2-aminobenzyl)-5-bromo-1-propyl-1,3-dihydroindol-2-one (0.031 g, $0.087 \mathrm{mmol}, 1 \mathrm{eq})$ in $\mathrm{AcOH}(2.0 \mathrm{ml})$ and $\mathrm{EtOH}(2.0 \mathrm{ml})$ was heated under reflux for 17 hours. The reaction mixture was allowed to cool to room temperature before dilution with water (10 ml). The resulting solution was made basic with $\mathrm{Na}_{2} \mathrm{CO}_{3}(\sim 3 \mathrm{~g})$. The aqueous layer was then extracted with EtOAc $(3 \times 10 \mathrm{ml})$ and the organic layer dried $\left(\mathrm{MgSO}_{4}\right)$, filtered and concentrated in vacuo to give the crude product as a yellow solid which was purified by flash chromatography using 5\% ethyl acetate in petroleum ether to give $\mathbf{1 4}$ $(0.023 \mathrm{~g}, 0.068 \mathrm{mmol}, 78 \%)$ as a yellow solid (mpt $154-155{ }^{\circ} \mathrm{C}$ recrystallized from $\mathrm{CH}_{2} \mathrm{Cl}_{2}$ ).

${ }^{1} \mathrm{H}$ NMR $\left(300 \mathrm{MHz}, \mathrm{CDCl}_{3}\right) \delta 1.01\left(3 \mathrm{H}, \mathrm{t}, \mathrm{J}=7.4 \mathrm{~Hz}, \mathrm{CH}_{3}\right), 1.92-2.04\left(2 \mathrm{H}, \mathrm{m}, \mathrm{CH}_{2} \mathrm{CH}_{3}\right)$, $4.47\left(2 \mathrm{H}, \mathrm{t}, \mathrm{J}=7.4 \mathrm{~Hz}, \mathrm{CH}_{2} \mathrm{~N}\right), 7.31(1 \mathrm{H}, \mathrm{d}, \mathrm{J}=8.4 \mathrm{~Hz}, \operatorname{ArCH}), 7.47(1 \mathrm{H}, \mathrm{ddd}, \mathrm{J}=1.4$, 6.8, 8.4 Hz, ArCH), $7.64(1 \mathrm{H}, \mathrm{dd}, \mathrm{J}=2.0,8.4 \mathrm{~Hz}, \operatorname{ArCH}), 7.74(1 \mathrm{H}, \mathrm{ddd}, 1.4,6.8,8.4 \mathrm{~Hz}$, $\operatorname{ArCH}), 7.99(1 \mathrm{H}, \mathrm{dd}, \mathrm{J}=1.4,8.4 \mathrm{~Hz}, \operatorname{ArCH}), 8.12(1 \mathrm{H}, \mathrm{d}, \mathrm{J}=9.0 \mathrm{~Hz}, \operatorname{ArCH}), 8.26(1 \mathrm{H}$, $\mathrm{d}, \mathrm{J}=2.0 \mathrm{~Hz}, \operatorname{ArCH}), 8.68(1 \mathrm{H}, \mathrm{s}, \operatorname{ArCH})$.

${ }^{13} \mathrm{C}$ NMR $\left(75 \mathrm{MHz}, \mathrm{CDCl}_{3}\right) \delta 11.9\left(\mathrm{CH}_{3}\right), 22.1\left(\mathrm{CH}_{2} \mathrm{CH}_{3}\right), 43.4\left(\mathrm{CH}_{2} \mathrm{~N}\right), 110.8(\mathrm{ArCH})$, 112.6 (ArCH), $117.2(\operatorname{ArC}), 121.7(\operatorname{ArC}), 122.5(\operatorname{ArC}), 123.4(\operatorname{ArCH}), 124.4(\mathrm{ArCH})$, 124.5 ( $\mathrm{ArCH}), 128.0(\mathrm{ArCH}), 128.8(\mathrm{ArCH}), 129.4(\mathrm{ArCH}), 130.8(\mathrm{ArC}), 141.3(\mathrm{ArC})$, $147.4(\mathrm{ArC}), 152.7(\mathrm{ArC})$.

IR $v_{\max } /\left(\mathrm{cm}^{-1}\right): 3429,1653,1265,442$.

MS $m / z$ ( $\mathrm{ES}^{+}$mode): 339 (M+ $\mathrm{M}^{+}$H, 100 \%), 326 (24), 261 (80), 238 (12), 206 (19), 185 (14), 160 (37), 128 (22), 106 (31), 101 (27), 97 (18), 81 (15).

$\mathrm{C}_{18} \mathrm{H}_{16} \mathrm{~N}_{2} \mathrm{Br}$ requires 339.0491, found 339.0490 . 


\section{Azaspirocycle (15) and 3-(2-aminobenzyl)-5-bromo-3-hydroxy-1-propyl-1,3-}

dihydroindol-2-one (16)

15
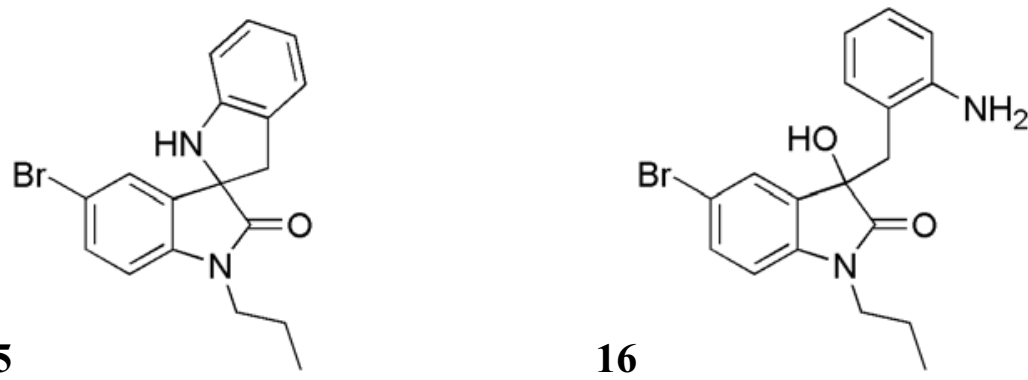

To a degassed solution of 5-bromo-3-(3,3,4,4,5,5,6,6,7,7,8,8,9,9,10,10,10heptadecafluorodecane-1-sulfonyl)-3-(2-nitrobenzyl)-1-propyl-1,3-dihydroindol-2-one $(0.214 \mathrm{~g}, 0.238 \mathrm{mmol}, 1 \mathrm{eq})$ in THF $(5 \mathrm{ml})$ was added $\mathrm{SmI}_{2}(33 \mathrm{ml}, 0.1 \mathrm{M}$ solution in THF, $3.33 \mathrm{mmol}, 14 \mathrm{eq}$ ) at room temperature and allowed to stir for 3.5 hours. Once yellow, the reaction mixture was quenched with aqueous saturated $\mathrm{NaHCO}_{3}(20 \mathrm{ml})$ and the aqueous layer extracted with EtOAc $(3 \times 10 \mathrm{ml})$. The organic layer was then dried $\left(\mathrm{MgSO}_{4}\right)$, filtered and concentrated in vacuo to give the crude product as a brown oil which was purified by flash chromatography using $15 \%$ ethyl acetate in petroleum ether to give $15(0.025 \mathrm{~g}, 0.071 \mathrm{mmol}, 30 \%)$ as a yellow solid (mpt 159.6-161.9 ${ }^{\circ} \mathrm{C}$ recrystallized from $\mathrm{CH}_{2} \mathrm{Cl}_{2}$ ) and 3-(2-aminobenzyl)-5-bromo-3-hydroxy-1-propyl-1,3dihydroindol-2-one $16(0.027 \mathrm{~g}, 0.071 \mathrm{mmol}, 30 \%)$ as a yellow oil.

For 15: ${ }^{1} \mathrm{H}$ NMR $\left(500 \mathrm{MHz}, \mathrm{CDCl}_{3}\right) \delta 0.98\left(3 \mathrm{H}, \mathrm{t}, \mathrm{J}=7.3 \mathrm{~Hz}, \mathrm{CH}_{3}\right), 1.61(1 \mathrm{H}$, broad s, $\mathrm{OH}), 1.69-1.76\left(2 \mathrm{H}, \mathrm{m}, \mathrm{CH}_{2} \mathrm{CH}_{3}\right), 3.26\left(1 \mathrm{H}, \mathrm{d}, \mathrm{J}=15.8 \mathrm{~Hz}, 1 \mathrm{H}\right.$ of $\left.\mathrm{CH}_{2} \mathrm{Ar}\right), 3.62(1 \mathrm{H}, \mathrm{d}, \mathrm{J}$ $=15.8 \mathrm{~Hz}, 1 \mathrm{H}$ of $\left.\mathrm{CH}_{2} \mathrm{Ar}\right), 3.63-3.74\left(2 \mathrm{H}, \mathrm{m}, \mathrm{CH}_{2} \mathrm{~N}\right), 4.20(1 \mathrm{H}$, broad s, $\mathrm{NH}), 6.75(2 \mathrm{H}$, $\mathrm{dd}, \mathrm{J}=8.6,11.9 \mathrm{~Hz}, 2 \times \operatorname{ArCH}), 6.84(1 \mathrm{H}, \mathrm{t}, \mathrm{J}=7.3 \mathrm{~Hz}, \operatorname{ArCH}), 7.13(2 \mathrm{H}, \mathrm{dd}, \mathrm{J}=7.3$, $11.9 \mathrm{~Hz}, 2 \times \operatorname{ArCH}), 7.36(1 \mathrm{H}, \mathrm{d}, \mathrm{J}=2.0 \mathrm{~Hz}, \operatorname{ArCH}), 7.42(1 \mathrm{H}, \mathrm{dd}, \mathrm{J}=2.0,8.6 \mathrm{~Hz}$, $\mathrm{ArCH})$.

${ }^{13} \mathrm{C}$ NMR $\left(75 \mathrm{MHz}, \mathrm{CDCl}_{3}\right) \delta 11.5\left(\mathrm{CH}_{3}\right), 20.8\left(\mathrm{CH}_{2} \mathrm{CH}_{3}\right), 42.0\left(\mathrm{CH}_{2} \mathrm{Ar}\right), 42.3\left(\mathrm{CH}_{2} \mathrm{~N}\right)$, $68.2(\mathrm{CAr}), 110.3(\mathrm{ArCH}), 110.3(\mathrm{ArCH}), 115.7(\mathrm{ArC}), 120.1(\mathrm{ArCH}), 124.9(\mathrm{ArCH})$, $126.3(\mathrm{ArCH}), 128.2(\mathrm{ArCH}), 132.3(\mathrm{ArCH}), 132.3(\mathrm{ArC}), 135.3(\mathrm{ArC}), 141.1(\mathrm{ArC})$, 149.6 (ArC), $178.0(C=\mathrm{O})$. 
IR $v_{\max } /\left(\mathrm{cm}^{-1}\right): 3390,3053,2305,1719,1607,1483,1350,1265,1111,895,737$.

MS m/z ( $\mathrm{CI}^{+}$mode): 357 (M+ H, 64 \%), 279 (98), 261 (7), 176 (8), 136 (14), 123 (12), 108 (42), 94 (31), 84 (34), 70 (25), 58 (100).

$\mathrm{C}_{18} \mathrm{H}_{18} \mathrm{ON}_{2} \mathrm{Br}$ requires 357.0597 , found 357.0593 .

For 16: ${ }^{1} \mathrm{H}$ NMR $\left(500 \mathrm{MHz}, \mathrm{CDCl}_{3}\right) \delta 0.82\left(3 \mathrm{H}, \mathrm{t}, \mathrm{J}=7.3 \mathrm{~Hz}, \mathrm{CH}_{3}\right), 1.32(1 \mathrm{H}$, broad s, $\mathrm{OH}), 1.50-1.57\left(2 \mathrm{H}, \mathrm{m}, \mathrm{CH}_{2} \mathrm{CH}_{3}\right), 2.92\left(1 \mathrm{H}, \mathrm{d}, \mathrm{J}=14.2 \mathrm{~Hz}, 1 \mathrm{H}\right.$ of $\left.\mathrm{CH}_{2} \mathrm{Ar}\right), 3.41(1 \mathrm{H}, \mathrm{d}, \mathrm{J}$ $=14.2 \mathrm{~Hz}, 1 \mathrm{H}$ of $\left.\mathrm{CH}_{2} \mathrm{Ar}\right), 3.41-3.45\left(1 \mathrm{H}, \mathrm{m}, 1 \mathrm{H}\right.$ of $\left.\mathrm{CH}_{2} \mathrm{~N}\right), 3.65-3.70(1 \mathrm{H}, \mathrm{m}, 1 \mathrm{H}$ of $\left.\mathrm{CH}_{2} \mathrm{~N}\right), 3.91\left(2 \mathrm{H}\right.$, broad s, $\left.\mathrm{NH}_{2}\right), 6.59(1 \mathrm{H}, \mathrm{dd}, \mathrm{J}=1.5,7.6 \mathrm{~Hz}, \mathrm{ArCH}), 6.65(1 \mathrm{H}, \mathrm{d}, \mathrm{J}=$ $8.4 \mathrm{~Hz}, \operatorname{ArCH}), 6.68(1 \mathrm{H}, \mathrm{d}, \mathrm{J}=6.6 \mathrm{~Hz}, \operatorname{ArCH}), 6.73(1 \mathrm{H}, \mathrm{d}, \mathrm{J}=7.6 \mathrm{~Hz}, \operatorname{ArCH}), 7.07$ $(1 \mathrm{H}, \mathrm{dt}, \mathrm{J}=1.5,7.6 \mathrm{~Hz}, \operatorname{ArCH}), 7.17(1 \mathrm{H}, \mathrm{d}, \mathrm{J}=1.9 \mathrm{~Hz}, \operatorname{ArCH}), 7.41(1 \mathrm{H}, \mathrm{dd}, \mathrm{J}=1.9,8.4$ $\mathrm{Hz}, \mathrm{ArCH})$.

${ }^{13} \mathrm{C}$ NMR $\left(126 \mathrm{MHz}, \mathrm{CDCl}_{3}\right)$ d $11.3\left(\mathrm{CH}_{3}\right), 20.6\left(\mathrm{CH}_{2} \mathrm{CH}_{3}\right), 39.9\left(\mathrm{CH}_{2} \mathrm{Ar}\right), 41.8\left(\mathrm{CH}_{2} \mathrm{~N}\right)$, $77.8(\mathrm{COH}), 110.2(\mathrm{ArCH}), 115.4(\mathrm{ArC}), 118.1(\mathrm{ArCH}), 119.8(\mathrm{ArCH}), 120.9(\mathrm{ArC})$, $128.3(\mathrm{ArCH}), 128.6(\mathrm{ArCH}), 132.4(\mathrm{ArCH}), 132.4(\mathrm{ArC}), 132.5(\mathrm{ArCH}), 141.7(\operatorname{ArC})$, $145.1(\mathrm{ArC}), 178.0(\mathrm{C}=\mathrm{O})$.

IR $v_{\max } /\left(\mathrm{cm}^{-1}\right): 3407,2305,1717,1609,1422,1265,896,737$.

MS m/z (CI mode): 375 (M+ $\mathrm{M}^{+}+\mathrm{H}, 5$ \%), 359 (4), 341 (8), 261 (6), 176 (3), 136 (3), 123

(5), 108 (100), 94 (15), 84 (18), 79 (13), 70 (18), 58 (60).

$\mathrm{C}_{18} \mathrm{H}_{20} \mathrm{O}_{2} \mathrm{~N}_{2} \mathrm{Br}$ requires 375.0703 , found 375.0709 .

3-(3,3,4,4,5,5,6,6,7,7,8,8,9,9,10,10,10-Heptadecafluorodecane-1-sulfonyl)-3-(2nitrobenzyl)-1-propyl-5-p-tolyl-1,3-dihydroindol-2-one (20)

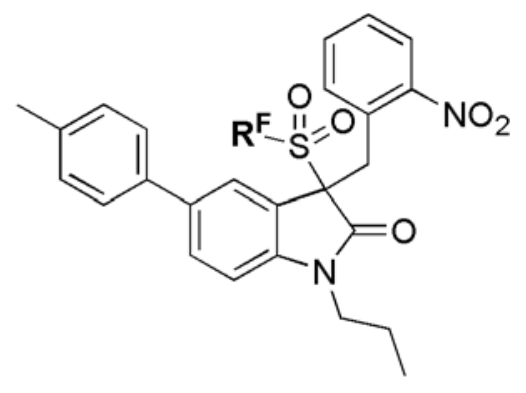


A solution of 5-bromo-3-(3,3,4,4,5,5,6,6,7,7,8,8,9,9,10,10,10-heptadecafluorodecane-1sulfonyl)-3-(2-nitrobenzyl)-1-propyl-1,3-dihydroindol-2-one (1.37 g, $1.52 \mathrm{mmol}, 1 \mathrm{eq})$, $\mathrm{Pd}\left(\mathrm{PPh}_{3}\right)_{4}(0.351 \mathrm{~g}, 0.304 \mathrm{mmol}, 0.2 \mathrm{eq})$ and 4-methyl benzene boronic acid $(0.619 \mathrm{~g}$, $4.56 \mathrm{mmol}, 3 \mathrm{eq})$ in aqueous saturated $\mathrm{Na}_{2} \mathrm{CO}_{3}(2.30 \mathrm{ml}, 4.56 \mathrm{mmol}, 2 \mathrm{M}, 3 \mathrm{eq}), \mathrm{H}_{2} \mathrm{O}$ (13 $\mathrm{ml})$ and 1,4-dioxane $(65 \mathrm{ml})$ was heated at $80{ }^{\circ} \mathrm{C}$ for 19 hours. After cooling to room temperature, the reaction mixture was diluted with $\mathrm{CH}_{2} \mathrm{Cl}_{2}(50 \mathrm{ml})$ and washed with $\mathrm{H}_{2} \mathrm{O}$ $(3 \times 30 \mathrm{ml})$. The organic layer was then dried $\left(\mathrm{MgSO}_{4}\right)$, filtered and concentrated in vacuo to give the crude product as a brown oil which was purified by fluorous chromatography to give $3-(3,3,4,4,5,5,6,6,7,7,8,8,9,9,10,10,10$-heptadecafluorodecane-1sulfonyl)-3-(2-nitrobenzyl)-1-propyl-5-p-tolyl-1,3-dihydroindol-2-one 20 (1.20 g, 1.32 mmol, $87 \%$ ) as a yellow solid (mpt $123.9-124.9{ }^{\circ} \mathrm{C}$ recrystallized from $\mathrm{CHCl}_{3}$ ).

${ }^{1} \mathrm{H}$ NMR $\left(500 \mathrm{MHz}, \mathrm{CDCl}_{3}\right) \delta 0.85\left(3 \mathrm{H}, \mathrm{t}, \mathrm{J}=7.4 \mathrm{~Hz}, \mathrm{CH}_{3} \mathrm{CH}_{2}\right), 1.51-1.59(2 \mathrm{H}, \mathrm{m}$, $\left.\mathrm{CH}_{2} \mathrm{CH}_{3}\right), 2.46\left(3 \mathrm{H}, \mathrm{s}, \mathrm{CH}_{3} \mathrm{ArC}\right), 2.68-2.87\left(2 \mathrm{H}, \mathrm{m}, \mathrm{CH}_{2} \mathrm{CF}_{2}\right), 3.53-3.62(2 \mathrm{H}, \mathrm{m}, 1 \mathrm{H}$ of $\mathrm{CH}_{2} \mathrm{~N}$ and $1 \mathrm{H}$ of $\left.\mathrm{CH}_{2} \mathrm{SO}_{2}\right), 3.66-3.72\left(1 \mathrm{H}, \mathrm{m}, 1 \mathrm{H}\right.$ of $\left.\mathrm{CH}_{2} \mathrm{~N}\right), 3.93-3.99(1 \mathrm{H}, \mathrm{m}, 1 \mathrm{H}$ of $\left.\mathrm{CH}_{2} \mathrm{SO}_{2}\right), 4.21\left(1 \mathrm{H}, \mathrm{d}, \mathrm{J}=13.6 \mathrm{~Hz}, 1 \mathrm{H}\right.$ of $\left.\mathrm{CH}_{2} \mathrm{Ar}\right), 4.70(1 \mathrm{H}, \mathrm{d}, \mathrm{J}=13.6 \mathrm{~Hz}, 1 \mathrm{H}$ of $\left.\mathrm{CH}_{2} \mathrm{Ar}\right), 6.84(1 \mathrm{H}, \mathrm{d}, \mathrm{J}=8.2 \mathrm{~Hz}, \operatorname{ArCH}), 7.31(2 \mathrm{H}, \mathrm{d}, \mathrm{J}=7.9 \mathrm{~Hz}, 2 \times \mathrm{ArCH}), 7.36(1 \mathrm{H}$, ddd, J = 1.9, 6.9, 8.2 Hz, ArCH), 7.44-7.48 (2H, m, $2 \times \operatorname{ArCH}), 7.52(2 \mathrm{H}, \mathrm{d}, \mathrm{J}=7.9 \mathrm{~Hz}, 2$ $\times \operatorname{ArCH}), 7.59(1 \mathrm{H}, \mathrm{dd}, \mathrm{J}=1.9,8.2 \mathrm{~Hz}, \operatorname{ArCH}), 7.76(1 \mathrm{H}, \mathrm{dd}, \mathrm{J}=1.3,8.2 \mathrm{~Hz}, \operatorname{ArCH})$, $7.80(1 \mathrm{H}, \mathrm{d}, \mathrm{J}=1.9 \mathrm{~Hz}, \mathrm{ArCH})$.

${ }^{13} \mathrm{C}$ NMR $\left(126 \mathrm{MHz}, \mathrm{CDCl}_{3}\right) \delta 11.2\left(\mathrm{CH}_{3} \mathrm{CH}_{2}\right), 20.7\left(\mathrm{CH}_{2} \mathrm{CH}_{3}\right), 21.1\left(\mathrm{CH}_{3} \mathrm{Ar}\right), 24.3$ $\left(\mathrm{CH}_{2} \mathrm{CF}_{2}, \mathrm{t}, \mathrm{J}=21.8 \mathrm{~Hz}\right), 32.9\left(\mathrm{CH}_{2} \mathrm{Ar}\right), 41.0\left(\mathrm{CH}_{2} \mathrm{SO}_{2}\right), 42.5\left(\mathrm{CH}_{2} \mathrm{~N}\right), 74.8\left(\mathrm{CSO}_{2}\right)$, 109.5 (ArCH), $120.0(\operatorname{ArC}), 125.4(\mathrm{ArCH}), 125.9(\mathrm{ArCH}), 126.9(2 \times \mathrm{ArCH}), 127.9$ (ArC), 129.1 (ArCH), $129.7(2 \times \operatorname{ArCH}), 129.9(\operatorname{ArC}), 133.0(\operatorname{ArC}), 133.7(\operatorname{ArC}), 137.2$ $(\mathrm{ArCH}), 137.3(\mathrm{ArCH}), 137.4(\mathrm{ArCH}), 143.2(\mathrm{ArC}), 149.9(\operatorname{ArC}), 169.9(C=\mathrm{O})$.

IR $v_{\max } /\left(\mathrm{cm}^{-1}\right): 3054,1710,1530,1488,1420,1265,739$.

MS $m / z$ ( $\mathrm{ES}^{+}$mode): 934 ( $\left.\mathrm{M}^{+}+\mathrm{Na}, 100 \%\right), 929\left(\mathrm{M}^{+}+\mathrm{H}, 40\right), 843$ (31), 745 (4), 523 (10), 198 (4), 160 (23).

$\mathrm{C}_{35} \mathrm{H}_{31} \mathrm{O}_{5} \mathrm{~N}_{3} \mathrm{~F}_{17} \mathrm{~S}$ requires 928.1707, found 928.1718. 


\section{5-(4-Fluorophenyl)-3-(3,3,4,4,5,5,6,6,7,7,8,8,9,9,10,10,10-heptadecafluorodecane-1-}

sulfonyl)-3-(2-nitrobenzyl)-1-propyl-1,3-dihydroindol-2-one (21)

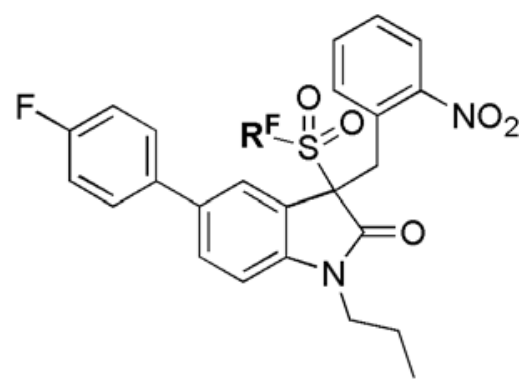

A solution of 5-bromo-3-(3,3,4,4,5,5,6,6,7,7,8,8,9,9,10,10,10-heptadecafluorodecane-1sulfonyl)-3-(2-nitrobenzyl)-1-propyl-1,3-dihydroindol-2-one (0.302 g, $0.336 \mathrm{mmol}, 1 \mathrm{eq})$, $\mathrm{Pd}\left(\mathrm{PPh}_{3}\right)_{4}(0.078 \mathrm{~g}, 0.067 \mathrm{mmol}, 0.2 \mathrm{eq})$ and 4-fluorobenzene boronic acid (0.141 g, 1.01 mmol, 3 eq) in aqueous saturated $\mathrm{Na}_{2} \mathrm{CO}_{3}(0.5 \mathrm{ml}, 1.01 \mathrm{mmol}, 2 \mathrm{M}, 3 \mathrm{eq}), \mathrm{H}_{2} \mathrm{O}(3 \mathrm{ml})$ and 1,4-dioxane $(15 \mathrm{ml})$ was heated at $80{ }^{\circ} \mathrm{C}$ for 4 hours. After cooling to room temperature, the reaction mixture was diluted with $\mathrm{CH}_{2} \mathrm{Cl}_{2}(20 \mathrm{ml})$ and washed with $\mathrm{H}_{2} \mathrm{O}$ $(3 \times 10 \mathrm{ml})$. The organic layer was then dried $\left(\mathrm{MgSO}_{4}\right)$, filtered and concentrated in vacuo to give the crude product as a brown oil which was purified by fluorous chromatography to give 5-(4-fluorophenyl)-3-(3,3,4,4,5,5,6,6,7,7,8,8,9,9,10,10,10heptadecafluorodecane-1-sulfonyl)-3-(2-nitrobenzyl)-1-propyl-1,3-dihydroindol-2-one 21 (0.242 $\mathrm{g}, 0.265 \mathrm{mmol}, 79 \%)$ as a white solid (mpt $157.1-159.1{ }^{\circ} \mathrm{C}$ recrystallized from $\left.\mathrm{CHCl}_{3}\right)$.

${ }^{1} \mathrm{H}$ NMR (500 MHz, $\left.\mathrm{CDCl}_{3}\right) \delta 0.79\left(3 \mathrm{H}, \mathrm{t}, \mathrm{J}=7.4 \mathrm{~Hz}, \mathrm{CH}_{3}\right), 1.44-1.51\left(2 \mathrm{H}, \mathrm{m}, \mathrm{CH}_{2} \mathrm{CH}_{3}\right)$, 2.61-2.76 (2H, m, $\left.\mathrm{CH}_{2} \mathrm{CF}_{2}\right), 3.45-3.53\left(2 \mathrm{H}, \mathrm{m}, 1 \mathrm{H}\right.$ of $\mathrm{CH}_{2} \mathrm{~N}$ and $1 \mathrm{H}$ of $\left.\mathrm{CH}_{2} \mathrm{SO}_{2}\right), 3.61-$ $3.67\left(1 \mathrm{H}, \mathrm{m}, 1 \mathrm{H}\right.$ of $\left.\mathrm{CH}_{2} \mathrm{~N}\right), 3.84-3.90\left(1 \mathrm{H}, \mathrm{m}, 1 \mathrm{H}\right.$ of $\left.\mathrm{CH}_{2} \mathrm{SO}_{2}\right), 4.07(1 \mathrm{H}, \mathrm{d}, \mathrm{J}=13.5 \mathrm{~Hz}$, $1 \mathrm{H}$ of $\left.\mathrm{CH}_{2} \mathrm{Ar}\right), 4.68\left(1 \mathrm{H}, \mathrm{d}, \mathrm{J}=13.5 \mathrm{~Hz}, 1 \mathrm{H}\right.$ of $\left.\mathrm{CH}_{2} \mathrm{Ar}\right), 6.79(1 \mathrm{H}, \mathrm{d}, \mathrm{J}=8.2 \mathrm{~Hz}, \mathrm{ArCH})$, $7.15(2 \mathrm{H}, \mathrm{t}, \mathrm{J}=8.8 \mathrm{~Hz}, 2 \times \operatorname{ArCH}), 7.34(1 \mathrm{H}, \mathrm{ddd}, \mathrm{J}=1.4,7.3,8.8 \mathrm{~Hz}, \operatorname{ArCH}), 7.41(1 \mathrm{H}$, $\mathrm{dd}, \mathrm{J}=1.4,8.2 \mathrm{~Hz}, \operatorname{ArCH}), 7.46(1 \mathrm{H}, \mathrm{td}, \mathrm{J}=7.3,1.4 \mathrm{~Hz}, \operatorname{ArCH}), 7.51-7.55$ (3H, m, 3 × $\operatorname{ArCH}), 7.67(1 \mathrm{H}, \mathrm{d}, \mathrm{J}=1.4 \mathrm{~Hz}, \operatorname{ArCH}), 7.72(1 \mathrm{H}, \mathrm{dd}, \mathrm{J}=1.4,8.2 \mathrm{~Hz}, \operatorname{ArCH})$. 
${ }^{13} \mathrm{C}$ NMR (126 MHz, $\left.\mathrm{CDCl}_{3}\right) \delta 11.4\left(\mathrm{CH}_{3}\right), 20.7\left(\mathrm{CH}_{2} \mathrm{CH}_{3}\right), 24.2\left(\mathrm{CH}_{2} \mathrm{CF}_{2}, \mathrm{t}, \mathrm{J}=25.4\right.$ $\mathrm{Hz}), 33.0\left(\mathrm{CH}_{2} \mathrm{Ar}\right), 41.0\left(\mathrm{CH}_{2} \mathrm{SO}_{2}\right), 42.6\left(\mathrm{CH}_{2} \mathrm{~N}\right), 74.8\left(\mathrm{CH}_{2} \mathrm{CSO}_{2}\right), 109.5(\mathrm{ArCH}), 115.9$ $(\mathrm{ArCH}), 116.0(\mathrm{ArCH}), 120.0(\mathrm{ArC}), 125.5(\mathrm{ArCH}), 126.1(\mathrm{ArCH}), 127.8(\mathrm{ArCH}), 128.9$ $(\mathrm{ArCH}), 129.1(\mathrm{ArCH}), 130.2(\mathrm{ArCH}), 133.0(\mathrm{ArCH}), 133.7(\mathrm{ArCH}), 136.2(\mathrm{ArC}), 136.6$ $(\mathrm{ArC}), 143.4(\mathrm{ArC}), 149.9(\mathrm{ArC}), 161.8(\mathrm{ArC}), 163.7(\mathrm{ArC}), 169.9(C=\mathrm{O})$.

IR $v_{\max } /\left(\mathrm{cm}^{-1}\right): 3052,1714,1420,1265,739$.

MS $m / z$ (ES mode): $937\left(\mathrm{M}^{+}+\mathrm{Na}, 45 \%\right), 932\left(\mathrm{M}^{+}+\mathrm{NH}_{4}, 13\right), 879$ (100), 843 (18), 450 (11), 337 (9).

$\mathrm{C}_{34} \mathrm{H}_{28} \mathrm{O}_{5} \mathrm{~N}_{3} \mathrm{~F}_{18} \mathrm{~S}$ requires 932.1457, found 932.1442.

\section{3-(3,3,4,4,5,5,6,6,7,7,8,8,9,9,10,10,10-Heptadecafluorodecane-1-sulfonyl)-3-(2-} nitrobenzyl)-1-propyl-5-thiophen-2-yl-1,3-dihydroindol-2-one (22)

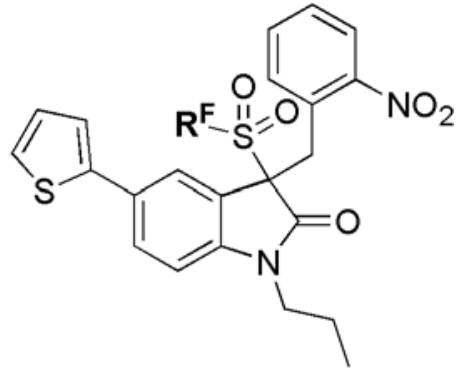

A solution of 5-bromo-3-(3,3,4,4,5,5,6,6,7,7,8,8,9,9,10,10,10-heptadecafluorodecane-1sulfonyl)-3-(2-nitrobenzyl)-1-propyl-1,3-dihydroindol-2-one (0.018 g, $0.019 \mathrm{mmol}, 1 \mathrm{eq})$, $\mathrm{Pd}\left(\mathrm{PPh}_{3}\right)_{4}(0.005 \mathrm{~g}, 0.004 \mathrm{mmol}, 0.2 \mathrm{eq})$ and thiophene-2-boronic acid (0.007 g, 0.058 mmol, 3 eq) in aqueous saturated $\mathrm{Na}_{2} \mathrm{CO}_{3}(0.03 \mathrm{ml}, 0.058 \mathrm{mmol}, 2 \mathrm{M}, 3 \mathrm{eq}), \mathrm{H}_{2} \mathrm{O}(0.2$ $\mathrm{ml})$ and 1,4-dioxane $(1 \mathrm{ml})$ was heated at $80{ }^{\circ} \mathrm{C}$ for 5 hours. After cooling to room temperature, the reaction mixture was diluted with $\mathrm{CH}_{2} \mathrm{Cl}_{2}(5 \mathrm{ml})$ and washed with $\mathrm{H}_{2} \mathrm{O}$ $(3 \times 10 \mathrm{ml})$. The organic layer was then dried $\left(\mathrm{MgSO}_{4}\right)$, filtered and concentrated in vacuo to give the crude product as a brown oil which was purified by fluorous chromatography to give $3-(3,3,4,4,5,5,6,6,7,7,8,8,9,9,10,10,10$-heptadecafluorodecane-1sulfonyl)-3-(2-nitrobenzyl)-1-propyl-5-thiophen-2-yl-1,3-dihydroindol-2-one 22 (0.017 g, $0.019 \mathrm{mmol}, 100 \%$ ) as a yellow solid (mpt $116.8-118^{\circ} \mathrm{C}$ recrystallized from $\mathrm{CHCl}_{3}$ ). 
${ }^{1} \mathrm{H}$ NMR $\left(500 \mathrm{MHz}, \mathrm{CDCl}_{3}\right) \delta 0.89\left(3 \mathrm{H}, \mathrm{t}, \mathrm{J}=7.4 \mathrm{~Hz}, \mathrm{CH}_{3}\right), 1.54-1.63\left(2 \mathrm{H}, \mathrm{m}, \mathrm{CH}_{2} \mathrm{CH}_{3}\right)$, 2.68-2.91 $\left(2 \mathrm{H}, \mathrm{m}, \mathrm{CH}_{2} \mathrm{CF}_{2}\right), 3.56-3.63\left(2 \mathrm{H}, \mathrm{m}, 1 \mathrm{H}\right.$ of $\mathrm{CH}_{2} \mathrm{SO}_{2}$ and $1 \mathrm{H}$ of $\left.\mathrm{CH}_{2} \mathrm{~N}\right), 3.69-$ $3.74\left(1 \mathrm{H}, \mathrm{m}, 1 \mathrm{H}\right.$ of $\left.\mathrm{CH}_{2} \mathrm{~N}\right), 3.93-3.99\left(1 \mathrm{H}, \mathrm{m}, 1 \mathrm{H}\right.$ of $\left.\mathrm{CH}_{2} \mathrm{SO}_{2}\right), 4.23(1 \mathrm{H}, \mathrm{d}, \mathrm{J}=13.5 \mathrm{~Hz}$, $1 \mathrm{H}$ of $\left.\mathrm{CH}_{2} \mathrm{Ar}\right), 4.67\left(1 \mathrm{H}, \mathrm{d}, \mathrm{J}=13.5 \mathrm{~Hz}, 1 \mathrm{H}\right.$ of $\left.\mathrm{CH}_{2} \mathrm{Ar}\right), 6.82(1 \mathrm{H}, \mathrm{d}, \mathrm{J}=8.2 \mathrm{~Hz}, \mathrm{ArCH})$, $7.16(1 \mathrm{H}, \mathrm{dd}, \mathrm{J}=3.5,5.1 \mathrm{~Hz}, \operatorname{ArCH}), 7.35-7.37(2 \mathrm{H}, \mathrm{m}, 2 \times \operatorname{ArCH}), 7.40(1 \mathrm{H}, \mathrm{td}, \mathrm{J}=7.7$, $1.3 \mathrm{~Hz}, \operatorname{ArCH}), 7.45(1 \mathrm{H}, \mathrm{dd}, \mathrm{J}=1.8,7.7 \mathrm{~Hz}, \operatorname{ArCH}), 7.51(1 \mathrm{H}, \mathrm{td}, \mathrm{J}=7.7,1.3 \mathrm{~Hz}$, $\operatorname{ArCH}), 7.63(1 \mathrm{H}, \mathrm{dd}, \mathrm{J}=1.8,8.2 \mathrm{~Hz}, \operatorname{ArCH}), 7.77(1 \mathrm{H}, \mathrm{d}, \mathrm{J}=1.8 \mathrm{~Hz}, \operatorname{ArCH}), 8.01(1 \mathrm{H}$, $\mathrm{dd}, \mathrm{J}=1.3,8.2 \mathrm{~Hz}, \mathrm{ArCH}$ ).

${ }^{13} \mathrm{C}$ NMR $\left(126 \mathrm{MHz}, \mathrm{CDCl}_{3}\right) \delta 11.3\left(\mathrm{CH}_{3}\right), 20.7\left(\mathrm{CH}_{2} \mathrm{CH}_{3}\right), 24.2\left(\mathrm{CH}_{2} \mathrm{CF}_{2}, \mathrm{t}, \mathrm{J}=22.7\right.$ $\mathrm{Hz}), 32.9\left(\mathrm{CH}_{2} \mathrm{Ar}\right), 41.0\left(\mathrm{CH}_{2} \mathrm{SO}_{2}\right), 42.6\left(\mathrm{CH}_{2} \mathrm{~N}\right), 74.6\left(\mathrm{CSO}_{2}\right), 109.6(\mathrm{ArCH}), 120.3$ ( $\mathrm{ArC}), 123.7(\mathrm{ArCH}), 124.9(\mathrm{ArCH}), 125.1(\mathrm{ArCH}), 125.5(\mathrm{ArCH}), 127.9(\mathrm{ArC}), 128.4$ ( $\mathrm{ArCH}), 129.1(\mathrm{ArCH}), 129.2(\mathrm{ArCH}), 130.8(\mathrm{ArC}), 133.1(\mathrm{ArCH}), 133.5(\mathrm{ArCH}), 143.2$ $(\operatorname{ArC}), 143.4(\operatorname{ArC}), 149.9(\operatorname{ArC}), 170.0(C=\mathrm{O})$.

IR $v_{\max } /\left(\mathrm{cm}^{-1}\right): 3433,1653,1265,738$.

MS $m / z$ (ES ${ }^{+}$mode): $920\left(\mathrm{M}^{+}+\mathrm{NH}_{4}, 91\right.$ \%), 879 (37), 838 (24), 531 (2), 449 (2), 337 (3), 273 (2), 241 (1), 160 (1).

$\mathrm{C}_{32} \mathrm{H}_{27} \mathrm{O}_{5} \mathrm{~N}_{3} \mathrm{~F}_{17} \mathrm{~S}_{2}$ requires 920.1115 , found 920.1117 .

\section{3-(2-Aminobenzyl)-1-propyl-5-p-tolyl-1,3-dihydroindol-2-one (23)}

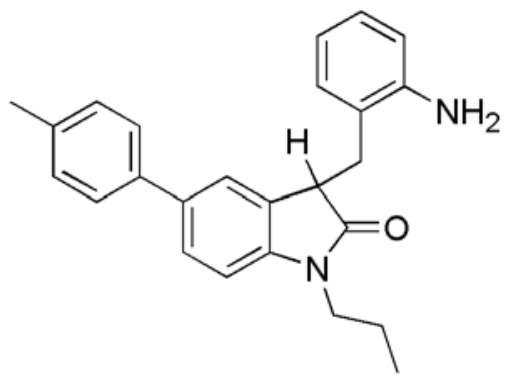

To a degassed solution of $3-(3,3,4,4,5,5,6,6,7,7,8,8,9,9,10,10,10$-heptadecafluorodecane1-sulfonyl)-3-(2-nitrobenzyl)-1-propyl-5-p-tolyl-1,3-dihydroindol-2-one (0.204 g, 0.224 mmol, 1 eq) in THF $(0.5 \mathrm{ml})$ was added $\mathrm{SmI}_{2}(40 \mathrm{ml}, 0.1 \mathrm{M}$ solution in THF, $4.04 \mathrm{mmol}$, 
18 eq) at room temperature and the reaction allowed to stir for 48 hours. The reaction was then quenched with aqueous saturated $\mathrm{NaHCO}_{3}(20 \mathrm{ml})$ and the aqueous layer extracted with EtOAc $(3 \times 10 \mathrm{ml})$. The organic layer was then dried $\left(\mathrm{MgSO}_{4}\right)$, filtered and concentrated in vacuo to give the crude product as a yellow oil which was purified by flash chromatography using $10 \%$ ethyl acetate in petroleum ether to give 3-(2aminobenzyl)-1-propyl-5-p-tolyl-1,3-dihydroindol-2-one 23 (0.042 g, 0.114 mmol, 51 \%, $77 \%$ based on recovered starting material) as a yellow oil.

${ }^{1} \mathrm{H}$ NMR $\left(500 \mathrm{MHz}, \mathrm{CDCl}_{3}\right) \delta 0.91\left(3 \mathrm{H}, \mathrm{t}, \mathrm{J}=7.5 \mathrm{~Hz}, \mathrm{CH}_{3} \mathrm{CH}_{2}\right), 1.63-1.71(2 \mathrm{H}, \mathrm{m}$, $\left.\mathrm{CH}_{2} \mathrm{CH}_{3}\right), 2.40\left(3 \mathrm{H}, \mathrm{s}, \mathrm{CH}_{3} \mathrm{Ar}\right), 2.94\left(1 \mathrm{H}, \mathrm{dd}, \mathrm{J}=8.9,14.4 \mathrm{~Hz}, 1 \mathrm{H}\right.$ of $\left.\mathrm{CH}_{2} \mathrm{Ar}\right), 3.40(1 \mathrm{H}$, $\mathrm{dd}, \mathrm{J}=4.0,14.4 \mathrm{~Hz}, 1 \mathrm{H}$ of $\left.\mathrm{CH}_{2} \mathrm{Ar}\right), 3.61-3.67\left(1 \mathrm{H}, \mathrm{m}, 1 \mathrm{H}\right.$ of $\left.\mathrm{CH}_{2} \mathrm{~N}\right), 3.70-3.77(1 \mathrm{H}, \mathrm{m}$, $1 \mathrm{H}$ of $\left.\mathrm{CH}_{2} \mathrm{~N}\right), 3.88(1 \mathrm{H}, \mathrm{dd}, \mathrm{J}=4.0,8.9 \mathrm{~Hz}, \mathrm{CH}), 4.03\left(2 \mathrm{H}\right.$, broad s, $\left.\mathrm{NH} \mathrm{H}_{2}\right), 6.67(1 \mathrm{H}, \mathrm{dt}, \mathrm{j}$ $=1.2,7.5 \mathrm{~Hz}, \operatorname{ArCH}), 6.72(1 \mathrm{H}, \mathrm{dd}, \mathrm{J}=1.2,7.8 \mathrm{~Hz}, \operatorname{ArCH}), 6.87(1 \mathrm{H}, \mathrm{d}, \mathrm{J}=8.3 \mathrm{~Hz}$, $\operatorname{ArCH}), 6.95(1 \mathrm{H}, \mathrm{dd}, \mathrm{J}=1.2,7.5 \mathrm{~Hz}, \operatorname{ArCH}), 7.08(1 \mathrm{H}, \mathrm{dt}, \mathrm{J}=1.8,7.8 \mathrm{~Hz}, \operatorname{ArCH}), 7.13$ $(1 \mathrm{H}, \mathrm{s}, \operatorname{ArCH}), 7.24(2 \mathrm{H}, \mathrm{d}, \mathrm{J}=8.3 \mathrm{~Hz}, 2 \times \operatorname{ArCH}), 7.38(2 \mathrm{H}, \mathrm{d}, \mathrm{J}=8.3 \mathrm{~Hz}, 2 \times \operatorname{ArCH})$, $7.47(1 \mathrm{H}, \mathrm{dd}, \mathrm{J}=1.8,8.3 \mathrm{~Hz}, \mathrm{ArCH})$.

${ }^{13} \mathrm{C}$ NMR $\left(126 \mathrm{MHz}, \mathrm{CDCl}_{3}\right) \delta 11.3\left(\mathrm{CH}_{3} \mathrm{CH}_{2}\right), 20.8\left(\mathrm{CH}_{2} \mathrm{CH}_{3}\right), 21.1\left(\mathrm{CH}_{3} \mathrm{Ar}\right), 33.0$ $\left(\mathrm{CH}_{2} \mathrm{Ar}\right), 41.7\left(\mathrm{CH}_{2} \mathrm{~N}\right), 45.0(\mathrm{CH}), 108.5(\mathrm{ArCH}), 116.2(\mathrm{ArCH}), 118.3(\mathrm{ArCH}), 122.1$ $(\mathrm{ArC}), 122.8(\mathrm{ArCH}), 123.6(\mathrm{ArCH}), 126.4(\mathrm{ArC}), 126.6(2 \times \mathrm{ArCH}), 128.1(\mathrm{ArCH})$, $129.5(2 \times \mathrm{ArCH}), 131.4(\mathrm{ArCH}), 135.3(\mathrm{ArC}), 136.7(\mathrm{ArC}), 138.0(\mathrm{ArC}), 142.8(\operatorname{ArC})$, $145.0(\operatorname{ArC}), 178.1(C=\mathrm{O})$.

IR $v_{\max } /\left(\mathrm{cm}^{-1}\right): 3396,2087,1652,1014$.

MS m/z (ES mode): $393\left(\mathrm{M}^{+}+\mathrm{Na}, 30 \%\right), 371\left(\mathrm{M}^{+}+\mathrm{H}, 100\right), 303$ (8), 281 (31).

$\mathrm{C}_{25} \mathrm{H}_{27} \mathrm{ON}_{2}$ requires 371.2118, found 371.2111.

\section{3-(2-Aminobenzyl)-5-(4-fluorophenyl)-1-propyl-1,3-dihydroindol-2-one (24)}




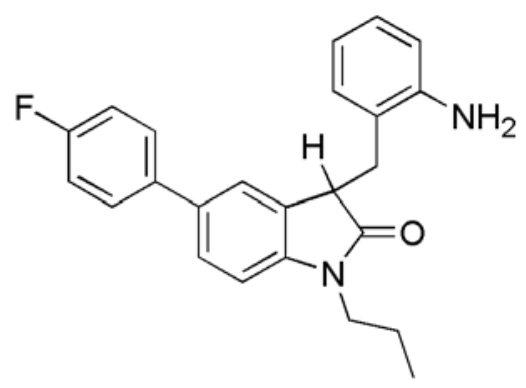

To a degassed solution of 5-(4-fluorophenyl)-3-(3,3,4,4,5,5,6,6,7,7,8,8,9,9,10,10,10heptadecafluorodecane-1-sulfonyl)-3-(2-nitrobenzyl)-1-propyl-1,3-dihydroindol-2-one $(0.266 \mathrm{~g}, 0.290 \mathrm{mmol}, 1 \mathrm{eq})$ in THF $(14 \mathrm{ml})$ was added $\mathrm{SmI}_{2}(41 \mathrm{ml}, 0.1 \mathrm{M}$ solution in THF, $4.07 \mathrm{mmol}, 14 \mathrm{eq}$ ) at room temperature and the reaction allowed to stir for 48 hours. The reaction was then quenched with aqueous saturated $\mathrm{NaHCO}_{3}(20 \mathrm{ml})$ and the aqueous layer extracted with EtOAc $(3 \times 10 \mathrm{ml})$. The organic layer was then dried $\left(\mathrm{MgSO}_{4}\right)$, filtered and concentrated in vacuo to give the crude product as a brown oil which was purified by flash chromatography using $10 \%$ ethyl acetate in petroleum ether to give 3-(2-aminobenzyl)-5-(4-fluorophenyl)-1-propyl-1,3-dihydroindol-2-one 24 (0.054 $\mathrm{g}, 0.145 \mathrm{mmol}, 50 \%, 63 \%$ based on recovered starting material) as a yellow oil.

${ }^{1} \mathrm{H}$ NMR $\left(500 \mathrm{MHz}, \mathrm{CDCl}_{3}\right) \delta 0.91\left(3 \mathrm{H}, \mathrm{t}, \mathrm{J}=7.4 \mathrm{~Hz}, \mathrm{CH}_{3}\right), 1.63\left(2 \mathrm{H}, \mathrm{m}, \mathrm{CH}_{2} \mathrm{CH}_{3}\right), 2.90$ $\left(1 \mathrm{H}, \mathrm{dd}, \mathrm{J}=9.3,14.3 \mathrm{~Hz}, 1 \mathrm{H}\right.$ of $\left.\mathrm{CH}_{2} \mathrm{CH}\right), 3.40\left(1 \mathrm{H}, \mathrm{dd}, \mathrm{J}=4.0,14.3 \mathrm{~Hz}, 1 \mathrm{H}\right.$ of $\left.\mathrm{CH}_{2} \mathrm{CH}\right)$, 3.61-3.67 $\left(1 \mathrm{H}, \mathrm{m}, 1 \mathrm{H}\right.$ of $\left.\mathrm{CH}_{2} \mathrm{~N}\right), 3.71-3.77\left(1 \mathrm{H}, \mathrm{m}, 1 \mathrm{H}\right.$ of $\left.\mathrm{CH}_{2} \mathrm{~N}\right), 3.88(1 \mathrm{H}, \mathrm{dd}, \mathrm{J}=4.0$, $9.3 \mathrm{~Hz}, \mathrm{CH}), 4.03\left(2 \mathrm{H}\right.$, broad s, $\left.\mathrm{NH}_{2}\right), 6.67(1 \mathrm{H}, \mathrm{td}, \mathrm{J}=7.8,1.3 \mathrm{~Hz}, \mathrm{ArCH}), 6.72(1 \mathrm{H}, \mathrm{dd}$, $\mathrm{J}=1.3,7.8 \mathrm{~Hz}, \operatorname{ArCH}), 6.87(1 \mathrm{H}, \mathrm{d}, \mathrm{J}=7.8 \mathrm{~Hz}, \operatorname{ArCH}), 6.93(1 \mathrm{H}, \mathrm{dd}, \mathrm{J}=1.3,7.8 \mathrm{~Hz}$, $\operatorname{ArCH}), 7.04(1 \mathrm{H}, \mathrm{s}, \mathrm{ArCH}), 7.09(3 \mathrm{H}, \mathrm{t}, \mathrm{J}=8.7 \mathrm{~Hz}, 3 \times \operatorname{ArCH}), 7.38-7.44(3 \mathrm{H}, \mathrm{m}, 3 \times$ $\mathrm{ArCH})$.

${ }^{13} \mathrm{C}$ NMR $\left(126 \mathrm{MHz}, \mathrm{CDCl}_{3}\right) \delta 11.5\left(\mathrm{CH}_{3}\right), 21.1\left(\mathrm{CH}_{2} \mathrm{CH}_{3}\right), 33.3\left(\mathrm{CH}_{2} \mathrm{CH}\right), 41.9\left(\mathrm{CH}_{2} \mathrm{~N}\right)$, $45.0(\mathrm{CH}), 108.8(\mathrm{ArCH}), 115.8(\mathrm{ArCH}, \mathrm{d}, \mathrm{J}=20.9 \mathrm{~Hz}), 116.4(\mathrm{ArCH}), 118.5(\mathrm{ArCH})$, $119.5(\mathrm{ArCH}), 122.9(\mathrm{ArCH}), 123.9(\mathrm{ArCH}), 126.7(\mathrm{ArCH}), 128.4(\mathrm{ArCH}, \mathrm{d}, \mathrm{J}=16.3$ $\mathrm{Hz}), 129.9(\mathrm{ArC}), 131.6(\mathrm{ArCH}), 134.6(\operatorname{ArC}), 135.9(\mathrm{ArCH}), 137.2(\mathrm{ArCH}), 143.3$ $(\operatorname{ArC}), 145.2(\operatorname{ArC}), 161.4(\operatorname{ArC}), 163.4(\operatorname{ArC}), 178.2(C=\mathrm{O})$.

IR $v_{\max } /\left(\mathrm{cm}^{-1}\right): 3393,2097,1654,1014$. 
MS $m / z$ (ES mode): $397\left(\mathrm{M}^{+}+\mathrm{Na}, 100 \%\right), 375$ (22), 303 (9).

$\mathrm{C}_{24} \mathrm{H}_{24} \mathrm{ON}_{2} \mathrm{~F}$ requires 375.1867 , found 375.1872 .

\section{3-(2-Aminobenzyl)-1-propyl-5-thiophen-2-yl-1,3-dihydroindol-2-one (25)}

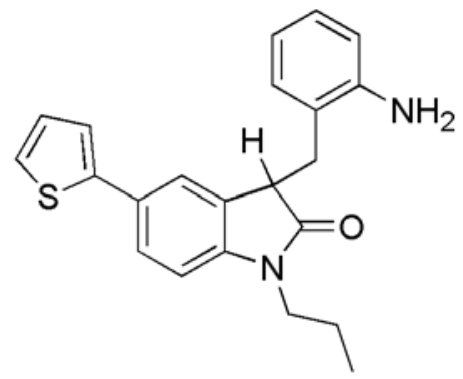

To a degassed solution of 3-(3,3,4,4,5,5,6,6,7,7,8,8,9,9,10,10,10-heptadecafluorodecane1-sulfonyl)-3-(2-nitrobenzyl)-1-propyl-5-thiophen-2-yl-1,3-dihydroindol-2-one (0.264 g, $0.293 \mathrm{mmol}, 1 \mathrm{eq})$ in THF $(14 \mathrm{ml})$ was added $\mathrm{SmI}_{2}(41 \mathrm{ml}, 0.1 \mathrm{M}$ solution in THF, 4.10 mmol, $14 \mathrm{eq}$ ) at room temperature and the reaction allowed to stir for 48 hours. The reaction was then quenched with aqueous saturated $\mathrm{NaHCO}_{3}(20 \mathrm{ml})$ and the aqueous layer extracted with EtOAc $(3 \times 10 \mathrm{ml})$. The organic layer was then dried $\left(\mathrm{MgSO}_{4}\right)$, filtered and concentrated in vacuo to give the crude product as a brown oil which was purified by flash chromatography using $10 \%$ ethyl acetate in petroleum ether to give 3(2-aminobenzyl)-1-propyl-5-thiophen-2-yl-1,3-dihydroindol-2-one 25 (0.052 g, 0.144 mmol, $49 \%, 73 \%$ based on recovered starting material) as a yellow oil.

${ }^{1} \mathrm{H}$ NMR $\left(500 \mathrm{MHz}, \mathrm{CDCl}_{3}\right) \delta 0.72\left(3 \mathrm{H}, \mathrm{t}, \mathrm{J}=7.4 \mathrm{~Hz}, \mathrm{CH}_{3}\right), 1.44-1.52\left(2 \mathrm{H}, \mathrm{m}, \mathrm{CH}_{2} \mathrm{CH}_{3}\right)$, $2.71\left(1 \mathrm{H}, \mathrm{dd}, \mathrm{J}=9.5,14.2 \mathrm{~Hz}, 1 \mathrm{H}\right.$ of $\left.\mathrm{CH}_{2} \mathrm{CH}\right), 3.21(1 \mathrm{H}, \mathrm{dd}, \mathrm{J}=4.1,14.2 \mathrm{~Hz}, 1 \mathrm{H}$ of $\left.\mathrm{CH}_{2} \mathrm{CH}\right), 3.42-3.47\left(1 \mathrm{H}, \mathrm{m}, 1 \mathrm{H}\right.$ of $\left.\mathrm{CH}_{2} \mathrm{~N}\right), 3.52-3.57\left(1 \mathrm{H}, \mathrm{m}, 1 \mathrm{H}\right.$ of $\left.\mathrm{CH}_{2} \mathrm{~N}\right), 3.68(1 \mathrm{H}, \mathrm{dd}$, $\mathrm{J}=4.1,9.5 \mathrm{~Hz}, \mathrm{CH}), 3.84\left(2 \mathrm{H}\right.$, broad s, $\left.\mathrm{NH}_{2}\right), 6.51(1 \mathrm{H}, \mathrm{td}, \mathrm{J}=4.1,9.5 \mathrm{~Hz}, \operatorname{ArCH}), 6.55$ $(1 \mathrm{H}, \mathrm{dd},=1.3,8.0 \mathrm{~Hz}, \operatorname{ArCH}), 6.63(1 \mathrm{H}, \mathrm{d}, \mathrm{J}=8.0 \mathrm{~Hz}, \operatorname{ArCH}), 6.75(1 \mathrm{H}, \mathrm{dd}, \mathrm{J}=1.6,7.6$ $\mathrm{Hz}, \mathrm{ArCH}), 6.87(1 \mathrm{H}, \mathrm{dd}, \mathrm{J}=3.8,5.0 \mathrm{~Hz}, \mathrm{ArCH}), 6.91-6.95(3 \mathrm{H}, \mathrm{m}, 3 \times \mathrm{ArCH}), 7.04$ $(1 \mathrm{H}, \mathrm{dd}, \mathrm{J}=1.3,5.0 \mathrm{~Hz}, \mathrm{ArCH}), 7.31(1 \mathrm{H}, \mathrm{ddd}, \mathrm{J}=0.7,1.9,8.0 \mathrm{~Hz}, \operatorname{ArCH})$. 
${ }^{13} \mathrm{C}$ NMR (126 MHz, $\left.\mathrm{CDCl}_{3}\right) \delta 11.2\left(\mathrm{CH}_{3}\right), 20.7\left(\mathrm{CH}_{2} \mathrm{CH}_{3}\right), 32.9\left(\mathrm{CH}_{2} \mathrm{CH}\right), 41.6\left(\mathrm{CH}_{2} \mathrm{~N}\right)$, $44.6(\mathrm{CH}), 108.5(\mathrm{ArCH}), 116.2(\mathrm{ArCH}), 118.3(\mathrm{ArCH}), 122.1(\mathrm{ArCH}), 122.5(\mathrm{ArC})$, 124.0 ( $\mathrm{ArCH}), 124.0(\mathrm{ArCH}), 125.5(\mathrm{ArCH}), 127.9(\mathrm{ArCH}), 128.0(\mathrm{ArCH}), 128.6(\mathrm{ArC})$, 129.6 (ArC), $131.3(\mathrm{ArCH}), 143.0(\operatorname{ArC}), 144.3(\operatorname{ArC}), 144.8(\operatorname{ArC}), 177.7(C=\mathrm{O})$.

IR $v_{\max } /\left(\mathrm{cm}^{-1}\right): 3341,2165,1652,1410,1023$.

MS $m / z$ (ES mode): $385\left(\mathrm{M}^{+}+\mathrm{Na}, 22 \%\right), 363\left(\mathrm{M}^{+}+\mathrm{H}, 100\right), 281(30)$.

$\mathrm{C}_{22} \mathrm{H}_{23} \mathrm{ON}_{2} \mathrm{~S}$ requires 363.1526 , found 363.1521 .

\section{9-(4-Methylphenyl)-6-propyl-6H-indolo[2,3-b]quinoline (26)}

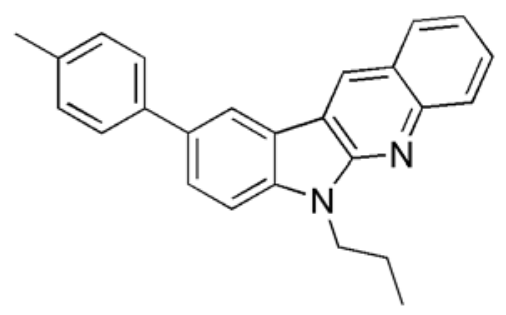

A solution of 3-(2-aminobenzyl)-1-propyl-5-p-tolyl-1,3-dihydroindol-2-one (0.030 g, $0.081 \mathrm{mmol}, 1 \mathrm{eq})$ in $\mathrm{AcOH}(2.0 \mathrm{ml})$ and $\mathrm{EtOH}(2.0 \mathrm{ml})$ was heated under reflux for 17 hours. The reaction mixture was allowed to cool to room temperature before dilution with water $(10 \mathrm{ml})$. The resulting solution was made basic with $\mathrm{Na}_{2} \mathrm{CO}_{3}(\sim 3 \mathrm{~g})$. The aqueous layer was then extracted with EtOAc $(3 \times 10 \mathrm{ml})$ and the organic layer dried $\left(\mathrm{MgSO}_{4}\right)$, filtered and concentrated in vacuo to give the crude product as a yellow solid which was purified by flash chromatography using 5\% ethyl acetate in petroleum ether to give $\mathbf{2 6}$ $(0.020 \mathrm{~g}, 0.057 \mathrm{mmol}, 70 \%)$ as a yellow solid (mpt $110.3-110.9{ }^{\circ} \mathrm{C}$ recrystallized from $\mathrm{CH}_{2} \mathrm{Cl}_{2}$ ).

${ }^{1} \mathrm{H}$ NMR $\left(500 \mathrm{MHz}, \mathrm{CDCl}_{3}\right) \delta 1.06\left(3 \mathrm{H}, \mathrm{t}, \mathrm{J}=7.4 \mathrm{~Hz}, \mathrm{CH}_{3} \mathrm{CH}_{2}\right), 2.00-2.07(2 \mathrm{H}, \mathrm{m}$, $\left.\mathrm{CH}_{2} \mathrm{CH}_{3}\right), 2.82\left(3 \mathrm{H}, \mathrm{s}, \mathrm{CH}_{3} \mathrm{Ar}\right), 4.53\left(2 \mathrm{H}, \mathrm{t}, \mathrm{J}=7.4 \mathrm{~Hz}, \mathrm{CH}_{2} \mathrm{~N}\right), 7.33(2 \mathrm{H}, \mathrm{d}, \mathrm{J}=8.2 \mathrm{~Hz}, 2$ $\times \mathrm{ArCH}), 7.47(1 \mathrm{H}, \mathrm{ddd}, \mathrm{J}=0.9,6.6,8.2 \mathrm{~Hz}, \mathrm{ArCH}), 7.52(1 \mathrm{H}, \mathrm{d}, \mathrm{J}=8.2 \mathrm{~Hz}, \operatorname{ArCH})$, $7.63(2 \mathrm{H}, \mathrm{dt}, \mathrm{J}=8.2,1.9 \mathrm{~Hz}, 2 \times \mathrm{ArCH}), 7.73(1 \mathrm{H}, \mathrm{ddd}, \mathrm{J}=1.9,6.6,8.2 \mathrm{~Hz}, \mathrm{ArCH}), 7.82$ 
$(1 \mathrm{H}, \mathrm{dd}, \mathrm{J}=1.9,8.2 \mathrm{~Hz}, \operatorname{ArCH}), 8.04(1 \mathrm{H}, \mathrm{dd}, \mathrm{J}=1.9,8.2 \mathrm{~Hz}, \operatorname{ArCH}), 8.16(1 \mathrm{H}, \mathrm{d}, \mathrm{J}=$ $8.2 \mathrm{~Hz}, \mathrm{ArCH}), 8.37(1 \mathrm{H}, \mathrm{d}, \mathrm{J}=1.9 \mathrm{~Hz}, \mathrm{ArCH}), 8.78(1 \mathrm{H}, \mathrm{s}, \mathrm{ArCH})$.

${ }^{13} \mathrm{C}$ NMR (126 MHz, $\left.\mathrm{CDCl}_{3}\right) \delta 11.9\left(\mathrm{CH}_{3} \mathrm{CH}_{2}\right), 21.3\left(\mathrm{CH}_{3} \mathrm{Ar}\right), 22.1\left(\mathrm{CH}_{2} \mathrm{CH}_{3}\right), 43.4$ $\left(\mathrm{CH}_{2} \mathrm{~N}\right), 109.5(\mathrm{ArCH}), 118.5(\mathrm{ArC}), 120.1(\mathrm{ArCH}), 121.2(\mathrm{ArC}), 123.1(\mathrm{ArCH}), 124.4$ $(\mathrm{ArC}), 127.3(2 \times \mathrm{ArCH}), 127.4(\mathrm{ArCH}), 127.6(\mathrm{ArCH}), 127.9(\mathrm{ArCH}), 128.8(\mathrm{ArCH})$, 129.0 (ArCH), $129.8(2 \times \mathrm{ArCH}), 133.5(\operatorname{ArC}), 136.7(\operatorname{ArC}), 138.9(\operatorname{ArC}), 141.9(\operatorname{ArC})$, $147.2(\operatorname{ArC}), 153.1(\operatorname{ArC})$.

IR $v_{\max } /\left(\mathrm{cm}^{-1}\right): 3435,2123,1645,1264,738$.

MS $m / z$ ( $\mathrm{ES}^{+}$mode): 351 ( $\left.\mathrm{M}^{+}+\mathrm{H}, 100 \%\right), 281$ (8), 261 (11), 194 (18).

$\mathrm{C}_{25} \mathrm{H}_{23} \mathrm{~N}_{2}$ requires 351.1856, found 351.1853 .

\section{9-(4-Fluorophenyl)-6-propyl-6H-indolo[2,3-b]quinoline (27)}

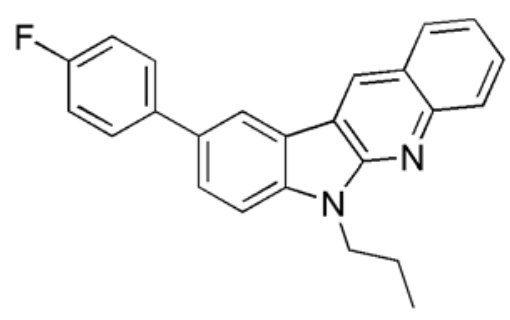

A solution of 3-(2-aminobenzyl)-5-(4-fluorophenyl)-1-propyl-1,3-dihydroindol-2-one $(0.038 \mathrm{~g}, 0.102 \mathrm{mmol}, 1 \mathrm{eq})$ in $\mathrm{AcOH}(2.0 \mathrm{ml})$ and $\mathrm{EtOH}(2.0 \mathrm{ml})$ was heated under reflux for 17 hours. The reaction mixture was allowed to cool to room temperature before dilution with water $(10 \mathrm{ml})$. The resulting solution was made basic with $\mathrm{Na}_{2} \mathrm{CO}_{3}(\sim 3 \mathrm{~g})$. The aqueous layer was then extracted with EtOAc $(3 \times 10 \mathrm{ml})$ and the organic layer dried $\left(\mathrm{MgSO}_{4}\right)$, filtered and concentrated in vacuo to give the crude product as a yellow oil which was purified by flash chromatography using 5\% ethyl acetate in petroleum ether to give $27(0.026 \mathrm{~g}, 0.074 \mathrm{mmol}, 73 \%)$ as a yellow solid (mpt $148.0-150.3{ }^{\circ} \mathrm{C}$ recrystallized from $\mathrm{CH}_{2} \mathrm{Cl}_{2}$ ).

${ }^{1} \mathrm{H}$ NMR (500 MHz, $\left.\mathrm{CDCl}_{3}\right) \delta 1.06\left(3 \mathrm{H}, \mathrm{t}, \mathrm{J}=7.3 \mathrm{~Hz}, \mathrm{CH}_{3}\right), 2.00-2.08\left(2 \mathrm{H}, \mathrm{m}, \mathrm{CH}_{2} \mathrm{CH}_{3}\right)$, $4.54\left(2 \mathrm{H}, \mathrm{t}, \mathrm{J}=7.3 \mathrm{~Hz}, \mathrm{CH}_{2} \mathrm{~N}\right), 7.20(2 \mathrm{H}, \mathrm{tt}, \mathrm{J}=1.9,8.5 \mathrm{~Hz}, 2 \times \operatorname{ArCH}), 7.48(1 \mathrm{H}, \mathrm{ddd}, \mathrm{J}$ 
$=1.3,6.9,8.5 \mathrm{~Hz}, \operatorname{ArCH}), 7.50(1 \mathrm{H}, \mathrm{d}, \mathrm{J}=8.5 \mathrm{~Hz}, \operatorname{ArCH}), 7.67(2 \mathrm{H}, \mathrm{dd}, \mathrm{J}=5.4,8.8 \mathrm{~Hz}, 2$ $\times \mathrm{ArCH}), 7.75(2 \mathrm{H}, \mathrm{dt}, \mathrm{J}=1.9,8.5 \mathrm{~Hz}, 2 \times \mathrm{ArCH}), 8.03(1 \mathrm{H}, \mathrm{dd}, \mathrm{J}=1.3,7.9 \mathrm{~Hz}, \mathrm{ArCH})$, $8.16(1 \mathrm{H}, \mathrm{d}, \mathrm{J}=8.8 \mathrm{~Hz}, \operatorname{ArCH}), 8.33(1 \mathrm{H}, \mathrm{d}, \mathrm{J}=1.3 \mathrm{~Hz}, \operatorname{ArCH}), 8.78(1 \mathrm{H}, \mathrm{s}, \operatorname{ArCH})$.

${ }^{13} \mathrm{C} \mathrm{NMR}\left(126 \mathrm{MHz}, \mathrm{CDCl}_{3}\right) \delta 11.8\left(\mathrm{CH}_{3}\right), 22.0\left(\mathrm{CH}_{2} \mathrm{CH}_{3}\right), 43.3\left(\mathrm{CH}_{2} \mathrm{~N}\right), 109.5(\mathrm{ArCH})$, 115.7 ( $\mathrm{ArCH}), 115.9(\mathrm{ArCH}), 118.2(\mathrm{ArC}), 120.1(\mathrm{ArCH}), 121.1(\mathrm{ArC}), 123.1(\mathrm{ArCH})$, 124.3 ( $\mathrm{ArC}), 127.3(\mathrm{ArCH}), 127.5(\mathrm{ArCH}), 127.8(\mathrm{ArCH}), 128.7(\mathrm{ArCH}), 128.8(\mathrm{ArCH})$, $128.8(\mathrm{ArCH}), 129.0(\mathrm{ArCH}), 132.4(\mathrm{ArC}), 137.9(\mathrm{ArC}), 141.8(\mathrm{ArC}), 147.1(\mathrm{ArC})$, 153.0 (ArC), $162.5(\operatorname{ArC}, \mathrm{d}, \mathrm{J}=245.3 \mathrm{~Hz})$.

IR $v_{\max }\left(\mathrm{cm}^{-1}\right): 3341,2524,2045,1459,1271,1115,1032$.

MS $m / z$ ( $\mathrm{CI}^{+}$mode): $355\left(\mathrm{M}^{+}+\mathrm{H}, 100 \%\right), 311$ (2), 261 (2).

$\mathrm{C}_{24} \mathrm{H}_{20} \mathrm{~N}_{2} \mathrm{~F}$ requires 355.1605 , found 355.1597 .

\section{9-(2-Thienyl)-6-propyl-6H-indolo[2,3-b]quinoline (28)}

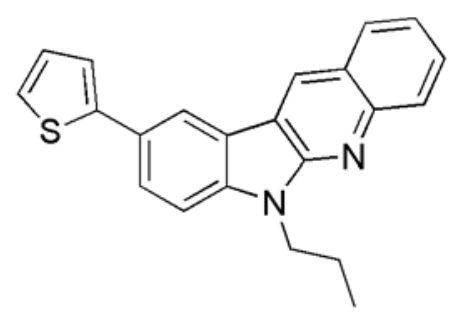

A solution of 3-(2-aminobenzyl)-1-propyl-5-thiophen-2-yl-1,3-dihydroindol-2-one (0.034 $\mathrm{g}, 0.094 \mathrm{mmol}, 1 \mathrm{eq})$ in $\mathrm{AcOH}(2.0 \mathrm{ml})$ and $\mathrm{EtOH}(2.0 \mathrm{ml})$ was heated under reflux for 17 hours. The reaction mixture was allowed to cool to room temperature before dilution with water $(10 \mathrm{ml})$. The resulting solution was made basic with $\mathrm{Na}_{2} \mathrm{CO}_{3}(\sim 3 \mathrm{~g})$. The aqueous layer was then extracted with EtOAc $(3 \times 10 \mathrm{ml})$ and the organic layer dried $\left(\mathrm{MgSO}_{4}\right)$, filtered and concentrated in vacuo to give the crude product as a yellow solid which was purified by flash chromatography using 5\% ethyl acetate in petroleum ether to give $\mathbf{2 8}$ $(0.025 \mathrm{~g}, 0.073 \mathrm{mmol}, 78 \%)$ as a yellow solid (mpt $96.4-98.6{ }^{\circ} \mathrm{C}$ recrystallized from $\left.\mathrm{CH}_{2} \mathrm{Cl}_{2}\right)$. 
${ }^{1} \mathrm{H}$ NMR (500 MHz, $\left.\mathrm{CDCl}_{3}\right) \delta 1.04\left(3 \mathrm{H}, \mathrm{t}, \mathrm{J}=7.4 \mathrm{~Hz}, \mathrm{CH}_{3}\right), 1.98-2.05\left(2 \mathrm{H}, \mathrm{m}, \mathrm{CH}_{2} \mathrm{CH}_{3}\right)$, $4.50\left(2 \mathrm{H}, \mathrm{t}, \mathrm{J}=7.4 \mathrm{~Hz}, \mathrm{CH}_{2} \mathrm{~N}\right), 7.14(1 \mathrm{H}, \mathrm{dd}, \mathrm{J}=3.5,5.2 \mathrm{~Hz}, \operatorname{ArCH}), 7.30(1 \mathrm{H}, \mathrm{dd}, \mathrm{J}=$ 1.1, $5.2 \mathrm{~Hz}, \operatorname{ArCH}), 7.37(1 \mathrm{H}, \mathrm{dd}, \mathrm{J}=1.1,3.5 \mathrm{~Hz}, \operatorname{ArCH}), 7.44(1 \mathrm{H}, \mathrm{d}, \mathrm{J}=8.8 \mathrm{~Hz}$, $\operatorname{ArCH}), 7.47(1 \mathrm{H}, \mathrm{ddd}, \mathrm{J}=1.3,6.9,8.2 \mathrm{~Hz}, \operatorname{ArCH}), 7.73(1 \mathrm{H}, \mathrm{ddd}, \mathrm{J}=1.3,6.9,8.2 \mathrm{~Hz}$, $\operatorname{ArCH}), 7.83(1 \mathrm{H}, \mathrm{dd}, \mathrm{J}=1.7,8.2 \mathrm{~Hz}, \operatorname{ArCH}), 8.02(1 \mathrm{H}, \mathrm{dd}, \mathrm{J}=1.3,8.2 \mathrm{~Hz}, \operatorname{ArCH}), 8.15$ $(1 \mathrm{H}, \mathrm{d}, \mathrm{J}=8.8 \mathrm{~Hz}, \operatorname{ArCH}), 8.37(1 \mathrm{H}, \mathrm{d}, \mathrm{J}=1.7 \mathrm{~Hz}, \operatorname{ArCH}), 8.76(1 \mathrm{H}, \mathrm{s}, \operatorname{ArCH})$.

${ }^{13} \mathrm{C}$ NMR $\left(126 \mathrm{MHz}, \mathrm{CDCl}_{3}\right) \delta 11.9\left(\mathrm{CH}_{3}\right), 22.1\left(\mathrm{CH}_{2} \mathrm{CH}_{3}\right), 43.4\left(\mathrm{CH}_{2} \mathrm{~N}\right), 109.6(\mathrm{ArCH})$, 118.2 (ArCH), 119.2 (ArCH), $121.2(\mathrm{ArC}), 122.5(\mathrm{ArCH}), 123.2(\mathrm{ArCH}), 124.2(\mathrm{ArCH})$, $124.4(\mathrm{ArC}), 126.5(\mathrm{ArCH}), 126.8(\mathrm{ArCH}), 127.8(\mathrm{ArCH}), 127.9(\mathrm{ArC}), 128.3(\mathrm{ArCH})$, $128.8(\mathrm{ArC}), 129.1(\mathrm{ArCH}), 142.0(\operatorname{ArC}), 145.4(\operatorname{ArC}), 147.0(\operatorname{ArC}), 153.0(\operatorname{ArC})$.

IR $v_{\max } /\left(\mathrm{cm}^{-1}\right): 3435,1644,1264,734$.

MS $m / z$ (ES mode): $343\left(\mathrm{M}^{+}+\mathrm{H}, 100 \%\right), 230$ (3), 191 (3).

$\mathrm{C}_{22} \mathrm{H}_{19} \mathrm{~N}_{2} \mathrm{~S}$ requires 343.1263 , found 343.1272 .

\section{Azaspirocycle (29) and 3-(2-aminobenzyl)-3-hydroxy-1-propyl-5-p-tolyl-1,3-} dihydroindol-2-one (30)

29

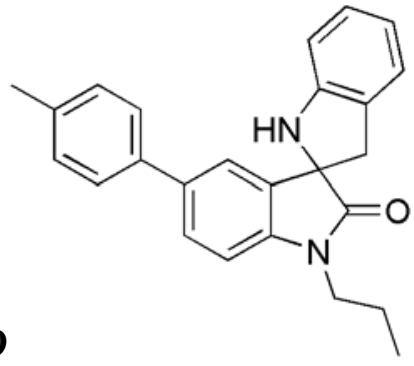

30

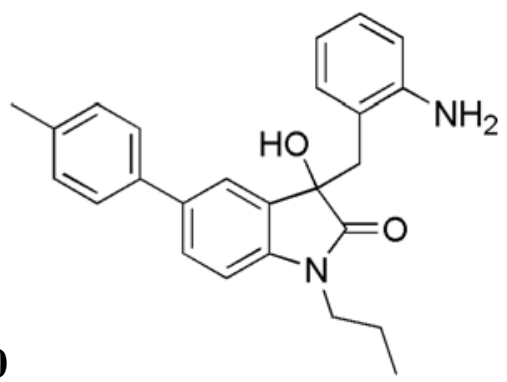

To a degassed solution of 3-(3,3,4,4,5,5,6,6,7,7,8,8,9,9,10,10,10-heptadecafluorodecane1-sulfonyl)-3-(2-nitrobenzyl)-1-propyl-5-p-tolyl-1,3-dihydroindol-2-one (0.176 g, 0.193 mmol, 1 eq) in THF $(8 \mathrm{ml})$ was added $\mathrm{SmI}_{2}(27 \mathrm{ml}, 0.1 \mathrm{M}$ solution in THF, $2.71 \mathrm{mmol}$, $14 \mathrm{eq}$ ) at room temperature and allowed to stir for 3.5 hours. Once yellow, the reaction mixture was quenched with aqueous saturated $\mathrm{NaHCO}_{3}(15 \mathrm{ml})$ and the aqueous layer extracted with EtOAc $(3 \times 10 \mathrm{ml})$. The organic layer was then dried $\left(\mathrm{MgSO}_{4}\right)$, filtered and concentrated in vacuo to give the crude product as a brown oil which was purified by 
flash chromatography using $20 \%$ ethyl acetate in petroleum ether to give $29(0.021 \mathrm{~g}$, $0.058 \mathrm{mmol}, 30 \%$ ) as a brown solid (mpt $149.5-150.8{ }^{\circ} \mathrm{C}$ recrystallized from $\mathrm{CHCl}_{3}$ ) and 3-(2-aminobenzyl)-3-hydroxy-1-propyl-5-p-tolyl-1,3-dihydroindol-2-one 30 (0.022 g, $0.058 \mathrm{mmol}, 30 \%$ ) as a brown solid (mpt $179.3-180.6{ }^{\circ} \mathrm{C}$ recrystallized from $\mathrm{CHCl}_{3}$ ).

For 29: ${ }^{1} \mathrm{H}$ NMR $\left(500 \mathrm{MHz}, \mathrm{CDCl}_{3}\right) \delta 1.02\left(3 \mathrm{H}, \mathrm{t}, \mathrm{J}=7.4 \mathrm{~Hz}, \mathrm{CH}_{3} \mathrm{CH}_{2}\right), 1.74-1.82(2 \mathrm{H}$, $\left.\mathrm{m}, \mathrm{CH}_{2} \mathrm{CH}_{3}\right), 2.38\left(3 \mathrm{H}, \mathrm{s}, \mathrm{CH}_{3} \mathrm{Ar}\right), 3.35\left(1 \mathrm{H}, \mathrm{d}, \mathrm{J}=15.9 \mathrm{~Hz}, 1 \mathrm{H}\right.$ of $\left.\mathrm{CH}_{2} \mathrm{Ar}\right), 3.65(1 \mathrm{H}, \mathrm{d}, \mathrm{J}$ $=15.9 \mathrm{~Hz}, 1 \mathrm{H}$ of $\left.\mathrm{CH}_{2} \mathrm{Ar}\right), 3.69-3.79\left(2 \mathrm{H}, \mathrm{m}, \mathrm{CH}_{2} \mathrm{~N}\right), 4.26(1 \mathrm{H}$, broad s, $\mathrm{N} H), 6.74(1 \mathrm{H}, \mathrm{d}$, $\mathrm{J}=7.9 \mathrm{~Hz}, \mathrm{ArCH}), 6.83(1 \mathrm{H}, \mathrm{td}, \mathrm{J}=7.2,1.0 \mathrm{~Hz}, \operatorname{ArCH}), 6.93(1 \mathrm{H}, \mathrm{d}, \mathrm{J}=8.2 \mathrm{~Hz}, \operatorname{ArCH})$, 7.10-7.15 (2H, m, $2 \times \operatorname{ArCH}), 7.20(2 \mathrm{H}, \mathrm{d}, \mathrm{J}=7.9 \mathrm{~Hz}, 2 \times \operatorname{ArCH}), 7.38(2 \mathrm{H}, \mathrm{d}, \mathrm{J}=8.2$ $\mathrm{Hz}, 2 \times \mathrm{ArCH}), 7.48(1 \mathrm{H}, \mathrm{d}, \mathrm{J}=1.9 \mathrm{~Hz}, \mathrm{ArCH}), 7.52(1 \mathrm{H}, \mathrm{dd}, \mathrm{J}=1.9,8.2 \mathrm{~Hz}, \mathrm{ArCH})$.

${ }^{13} \mathrm{C}$ NMR $\left(126 \mathrm{MHz}, \mathrm{CDCl}_{3}\right) \delta 11.6\left(\mathrm{CH}_{3} \mathrm{CH}_{2}\right), 21.0\left(\mathrm{CH}_{2} \mathrm{CH}_{3}\right), 21.3\left(\mathrm{CH}_{3} \mathrm{Ar}\right), 42.1$ $\left(\mathrm{CH}_{2} \mathrm{~N}\right), 42.4\left(\mathrm{CH}_{2} \mathrm{Ar}\right), 68.4(\mathrm{CNH}), 109.1(\mathrm{ArCH}), 110.2(\mathrm{ArCH}), 120.0(\mathrm{ArCH}), 121.9$ $(\mathrm{ArCH}), 124.9(\mathrm{ArCH}), 126.8(2 \times \mathrm{ArCH}), 128.1(\mathrm{ArCH}), 128.1(\mathrm{ArCH}), 129.7(2 \times$ $\operatorname{ArCH}), 134.0(\operatorname{ArC}), 136.8(\operatorname{ArC}), 137.2(\operatorname{ArC}), 137.8(\operatorname{ArC}), 141.3(\operatorname{ArC}), 150.0(\operatorname{ArC})$, $150.0(\mathrm{ArC}), 178.7(C=\mathrm{O})$.

IR $v_{\max } /\left(\mathrm{cm}^{-1}\right): 3053,2305,1718,1488,1265,896,739$.

MS m/z (ES mode): 391 (M+ Na, $100 \%$ \%), 301 (42), 205 (6), 145 (9).

$\mathrm{C}_{25} \mathrm{H}_{24} \mathrm{ON}_{2} \mathrm{Na}$ requires 391.1781 , found 391.1792 .

For 30: ${ }^{1} \mathrm{H}$ NMR $\left(500 \mathrm{MHz}, \mathrm{CDCl}_{3}\right) \delta 0.85\left(3 \mathrm{H}, \mathrm{t}, \mathrm{J}=7.4 \mathrm{~Hz}, \mathrm{CH}_{3} \mathrm{CH}_{2}\right), 1.54-1.62(2 \mathrm{H}$, m, $\left.\mathrm{CH}_{2} \mathrm{CH}_{3}\right), 2.36(1 \mathrm{H}, \mathrm{s}, \mathrm{OH}), 2.39\left(3 \mathrm{H}, \mathrm{s}, \mathrm{CH}_{3} \mathrm{Ar}\right), 2.97(1 \mathrm{H}, \mathrm{d}, \mathrm{J}=13.9 \mathrm{~Hz}, 1 \mathrm{H}$ of $\left.\mathrm{CH}_{2} \mathrm{Ar}\right), 3.44\left(1 \mathrm{H}, \mathrm{d}, \mathrm{J}=13.9 \mathrm{~Hz}, 1 \mathrm{H}\right.$ of $\left.\mathrm{CH}_{2} \mathrm{Ar}\right), 3.44-3.50\left(1 \mathrm{H}, \mathrm{m}, 1 \mathrm{H}\right.$ of $\left.\mathrm{CH}_{2} \mathrm{~N}\right), 3.69-$ $3.74\left(1 \mathrm{H}, \mathrm{m}, 1 \mathrm{H}\right.$ of $\left.\mathrm{CH}_{2} \mathrm{~N}\right), 4.01\left(2 \mathrm{H}\right.$, broad s, $\left.\mathrm{NH}_{2}\right), 6.62-6.63(2 \mathrm{H}, \mathrm{m}, 2 \times \mathrm{ArCH}), 6.69$ $(1 \mathrm{H}, \mathrm{d}, \mathrm{J}=7.9 \mathrm{~Hz}, \mathrm{ArCH}), 6.82(1 \mathrm{H}, \mathrm{d}, \mathrm{J}=7.9 \mathrm{~Hz}, \operatorname{ArCH}), 7.03-7.06(1 \mathrm{H}, \mathrm{m}, \operatorname{ArCH})$, $7.22(2 \mathrm{H}, \mathrm{d}, \mathrm{J}=9.2 \mathrm{~Hz}, 2 \times \operatorname{ArCH}), 7.30(1 \mathrm{H}, \mathrm{d}, \mathrm{J}=1.9 \mathrm{~Hz}, \operatorname{ArCH}), 7.38(2 \mathrm{H}, \mathrm{d}, \mathrm{J}=7.9$ $\mathrm{Hz}, 2 \times \mathrm{ArCH}), 7.49(1 \mathrm{H}, \mathrm{dd}, \mathrm{J}=1.9,7.9 \mathrm{~Hz}, \mathrm{ArCH})$.

${ }^{13} \mathrm{C}$ NMR $\left(126 \mathrm{MHz}, \mathrm{CDCl}_{3}\right) \delta 11.5\left(\mathrm{CH}_{3} \mathrm{CH}_{2}\right), 20.9\left(\mathrm{CH}_{2} \mathrm{CH}_{3}\right), 21.3\left(\mathrm{CH}_{3} \mathrm{Ar}\right), 40.1$ $\left(\mathrm{CH}_{2} \mathrm{Ar}\right), 41.9\left(\mathrm{CH}_{2} \mathrm{~N}\right), 78.0(\mathrm{COH}), 109.1(\mathrm{ArCH}), 117.8(\mathrm{ArC}), 119.4(\mathrm{ArCH}), 121.1$ $(\mathrm{ArCH}), 123.8(\mathrm{ArCH}), 126.7(2 \times \mathrm{ArCH}), 128.2(\mathrm{ArCH}), 128.5(\mathrm{ArCH}), 129.7(2 \times$ 
$\mathrm{ArCH}), 130.6(\mathrm{ArC}), 132.8(\mathrm{ArCH}), 136.0(\mathrm{ArC}), 137.0(\mathrm{ArC}), 137.9(\mathrm{ArC}), 141.9$

$(\operatorname{ArC}), 145.7(\operatorname{ArC}), 178.6(C=\mathrm{O})$.

IR $v_{\max } /\left(\mathrm{cm}^{-1}\right): 3438,1702,1618,1490,1265,1119,896$.

MS $m / z$ (ES ${ }^{+}$mode): 409 (M+ Na, $\left.100 \%\right), 391$ (19), 319 (21).

$\mathrm{C}_{25} \mathrm{H}_{26} \mathrm{O}_{2} \mathrm{~N}_{2} \mathrm{Na}$ requires 409.1886, found 409.1885. 


\section{X-ray crystallographic data for 8}

CCDC number for 8: 671492

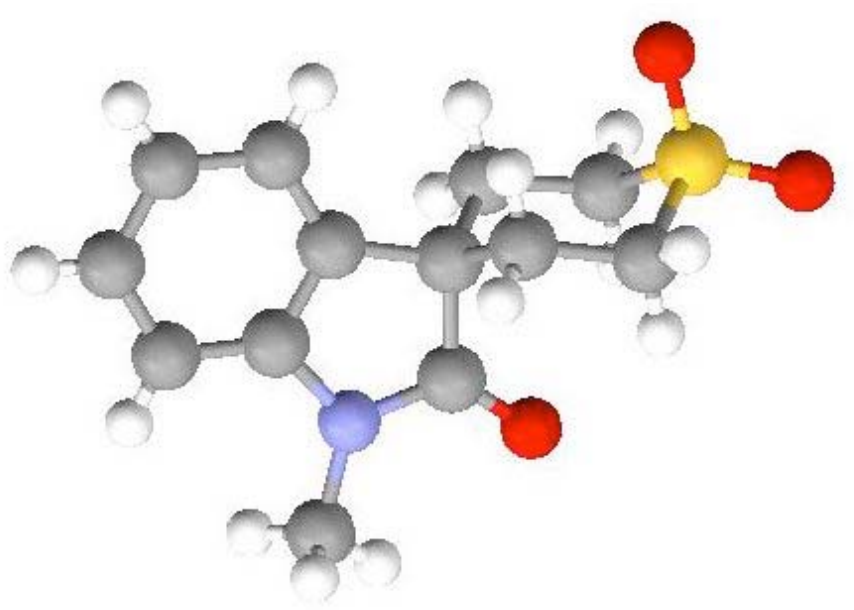


Table 1. Crystal data and structure refinement for s2812m.

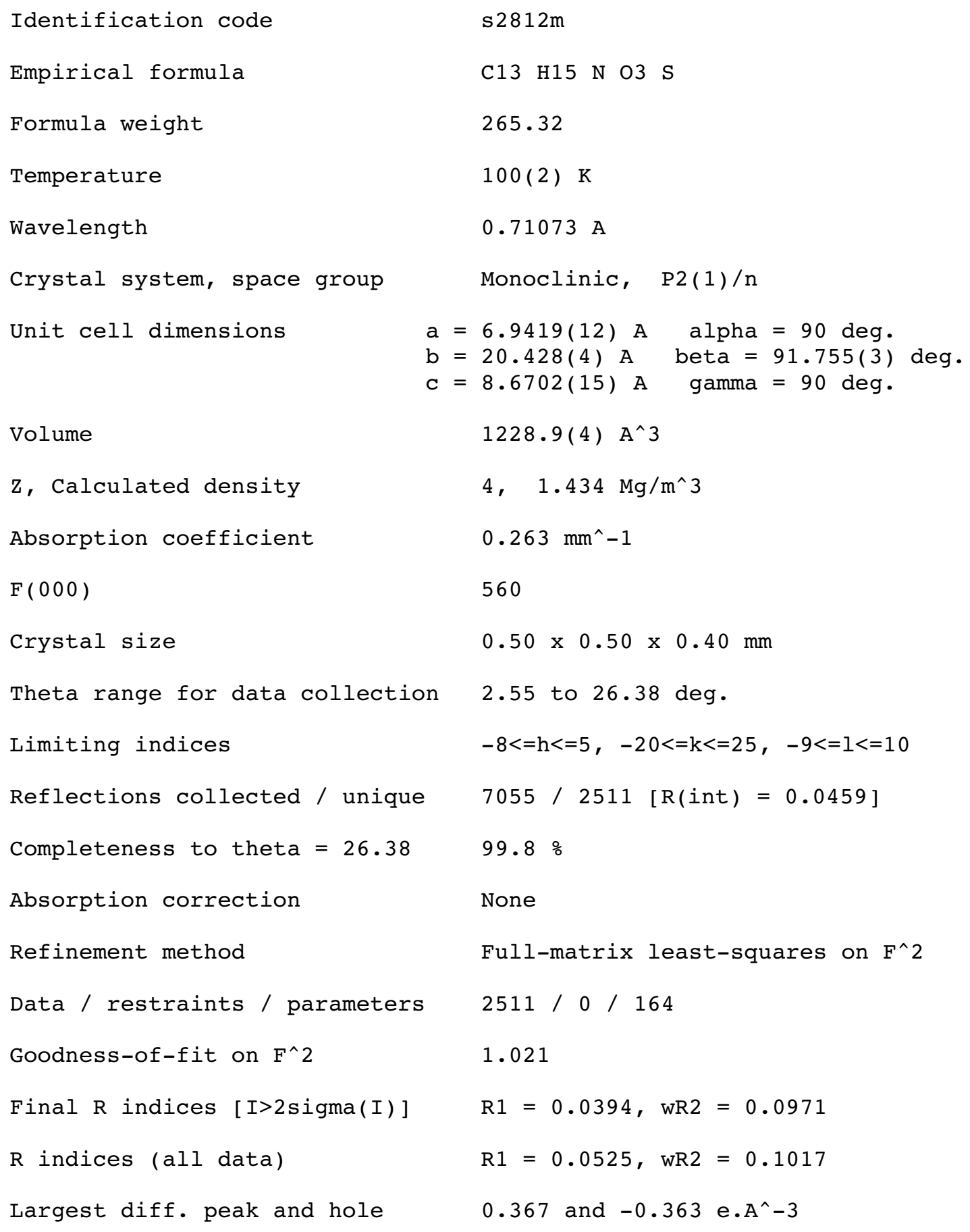


Table 2. Atomic coordinates ( $\left.\mathrm{x} 10^{\wedge} 4\right)$ and equivalent isotropic displacement parameters $\left(A^{\wedge} 2 \times 10^{\wedge} 3\right)$ for $52812 \mathrm{~m}$.

$\mathrm{U}(\mathrm{eq})$ is defined as one third of the trace of the orthogonalized Uij tensor.

\begin{tabular}{lrlll}
\hline & & & \\
& & & \\
& & & \\
& & & \\
& & & \\
S (1) & $8264(1)$ & $1928(1)$ & $4546(1)$ & $18(1)$ \\
$O(1)$ & $8167(2)$ & $3210(1)$ & $7862(1)$ & $18(1)$ \\
$O(2)$ & $8567(2)$ & $1303(1)$ & $5288(2)$ & $29(1)$ \\
$O(3)$ & $8065(2)$ & $1943(1)$ & $2885(2)$ & $22(1)$ \\
N $(1)$ & $7839(2)$ & $4272(1)$ & $7021(2)$ & $15(1)$ \\
$C(1)$ & $7957(2)$ & $3612(1)$ & $6818(2)$ & $14(1)$ \\
$C(2)$ & $7777(3)$ & $3466(1)$ & $5082(2)$ & $15(1)$ \\
$C(3)$ & $7512(3)$ & $4139(1)$ & $4408(2)$ & $15(1)$ \\
$C(4)$ & $7242(3)$ & $4342(1)$ & $2899(2)$ & $18(1)$ \\
$C(5)$ & $7087(3)$ & $5010(1)$ & $2593(2)$ & $19(1)$ \\
$C(6)$ & $7180(3)$ & $5462(1)$ & $3788(2)$ & $19(1)$ \\
$C(7)$ & $7430(3)$ & $5263(1)$ & $5323(2)$ & $15(1)$ \\
$C(8)$ & $7590(2)$ & $4599(1)$ & $5589(2)$ & $22(1)$ \\
$C(9)$ & $7953(3)$ & $4589(1)$ & $8524(2)$ & $17(1)$ \\
$C(10)$ & $6023(3)$ & $3019(1)$ & $4725(2)$ & $19(1)$ \\
$C(11)$ & $6238(3)$ & $2318(1)$ & $5322(2)$ & $17(1)$ \\
$C(12)$ & $10119(3)$ & $2475(1)$ & $5110(2)$ & \\
$C(13)$ & $9671(3)$ & $3161(1)$ & $4508(2)$ & \\
& & & & \\
\hline
\end{tabular}


Table 3. Bond lengths [A] and angles [deg] for s2812m.

\begin{tabular}{|c|c|}
\hline$S(1)-O(2)$ & $1.4421(14)$ \\
\hline$S(1)-O(3)$ & $1.4423(14)$ \\
\hline$S(1)-C(12)$ & $1.7637(19)$ \\
\hline$S(1)-C(11)$ & $1.7677(19)$ \\
\hline $\mathrm{O}(1)-\mathrm{C}(1)$ & $1.226(2)$ \\
\hline$N(1)-C(1)$ & $1.363(2)$ \\
\hline $\mathrm{N}(1)-\mathrm{C}(8)$ & $1.416(2)$ \\
\hline$N(1)-C(9)$ & $1.455(2)$ \\
\hline$C(1)-C(2)$ & $1.535(2)$ \\
\hline$C(2)-C(3)$ & $1.503(3)$ \\
\hline$C(2)-C(10)$ & $1.545(3)$ \\
\hline$C(2)-C(13)$ & $1.550(2)$ \\
\hline$C(3)-C(4)$ & $1.380(2)$ \\
\hline$C(3)-C(8)$ & $1.390(3)$ \\
\hline$C(4)-C(5)$ & $1.393(3)$ \\
\hline $\mathrm{C}(4)-\mathrm{H}(4)$ & 0.9500 \\
\hline$C(5)-C(6)$ & $1.387(3)$ \\
\hline $\mathrm{C}(5)-\mathrm{H}(5)$ & 0.9500 \\
\hline$C(6)-C(7)$ & $1.398(3)$ \\
\hline $\mathrm{C}(6)-\mathrm{H}(6)$ & 0.9500 \\
\hline$C(7)-C(8)$ & $1.379(3)$ \\
\hline $\mathrm{C}(7)-\mathrm{H}(7)$ & 0.9500 \\
\hline$C(9)-H(9 A)$ & 0.9800 \\
\hline$C(9)-H(9 B)$ & 0.9800 \\
\hline $\mathrm{C}(9)-\mathrm{H}(9 \mathrm{C})$ & 0.9800 \\
\hline$C(10)-C(11)$ & $1.527(3)$ \\
\hline$C(10)-H(10 A)$ & 0.9900 \\
\hline$C(10)-H(10 B)$ & 0.9900 \\
\hline$C(11)-H(11 A)$ & 0.9900 \\
\hline $\mathrm{C}(11)-\mathrm{H}(11 \mathrm{~B})$ & 0.9900 \\
\hline$C(12)-C(13)$ & $1.524(2)$ \\
\hline $\mathrm{C}(12)-\mathrm{H}(12 \mathrm{~A})$ & 0.9900 \\
\hline $\mathrm{C}(12)-\mathrm{H}(12 \mathrm{~B})$ & 0.9900 \\
\hline$C(13)-H(13 A)$ & 0.9900 \\
\hline$C(13)-H(13 B)$ & 0.9900 \\
\hline$O(2)-S(1)-O(3)$ & 118.25 ( 8 ) \\
\hline $\mathrm{O}(2)-\mathrm{S}(1)-\mathrm{C}(12)$ & $109.98(9)$ \\
\hline $\mathrm{O}(3)-\mathrm{S}(1)-\mathrm{C}(12)$ & $108.04(8)$ \\
\hline$O(2)-S(1)-C(11)$ & $109.67(9)$ \\
\hline$O(3)-S(1)-C(11)$ & $108.50(8)$ \\
\hline$C(12)-S(1)-C(11)$ & $100.99(9)$ \\
\hline$C(1)-N(1)-C(8)$ & $111.12(15)$ \\
\hline$C(1)-N(1)-C(9)$ & $123.67(16)$ \\
\hline $\mathrm{C}(8)-\mathrm{N}(1)-\mathrm{C}(9)$ & $125.20(16)$ \\
\hline $\mathrm{O}(1)-\mathrm{C}(1)-\mathrm{N}(1)$ & $124.90(17)$ \\
\hline $\mathrm{O}(1)-\mathrm{C}(1)-\mathrm{C}(2)$ & $126.72(17)$ \\
\hline$N(1)-C(1)-C(2)$ & $108.37(15)$ \\
\hline$C(3)-C(2)-C(1)$ & $102.06(14)$ \\
\hline$C(3)-C(2)-C(10)$ & $112.11(15)$ \\
\hline$C(1)-C(2)-C(10)$ & $110.55(14)$ \\
\hline$C(3)-C(2)-C(13)$ & $109.71(15)$ \\
\hline$C(1)-C(2)-C(13)$ & $110.37(14)$ \\
\hline$C(10)-C(2)-C(13)$ & $111.65(15)$ \\
\hline$C(4)-C(3)-C(8)$ & $119.77(18)$ \\
\hline
\end{tabular}




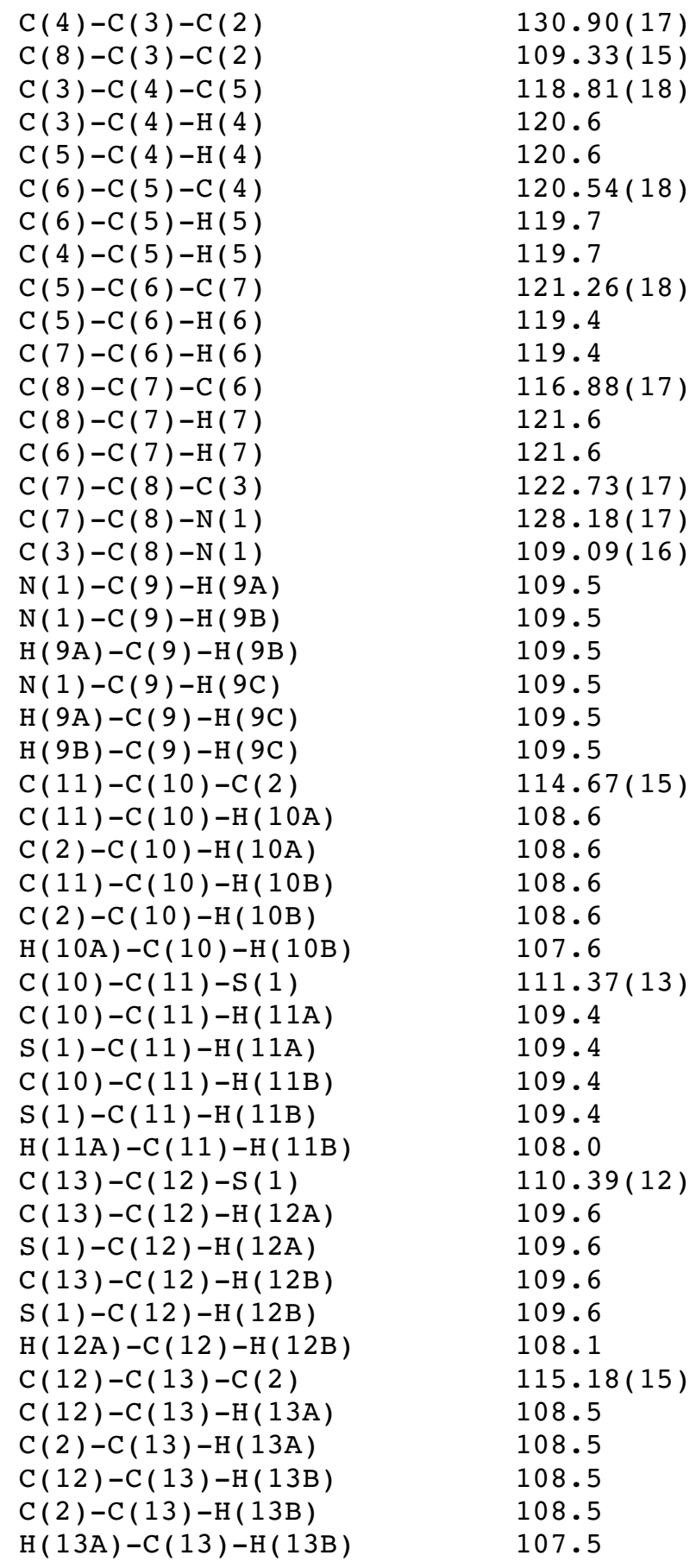

Symmetry transformations used to generate equivalent atoms: 


\section{${ }^{1} \mathrm{H}$ and ${ }^{13} \mathrm{C}$ spectra}
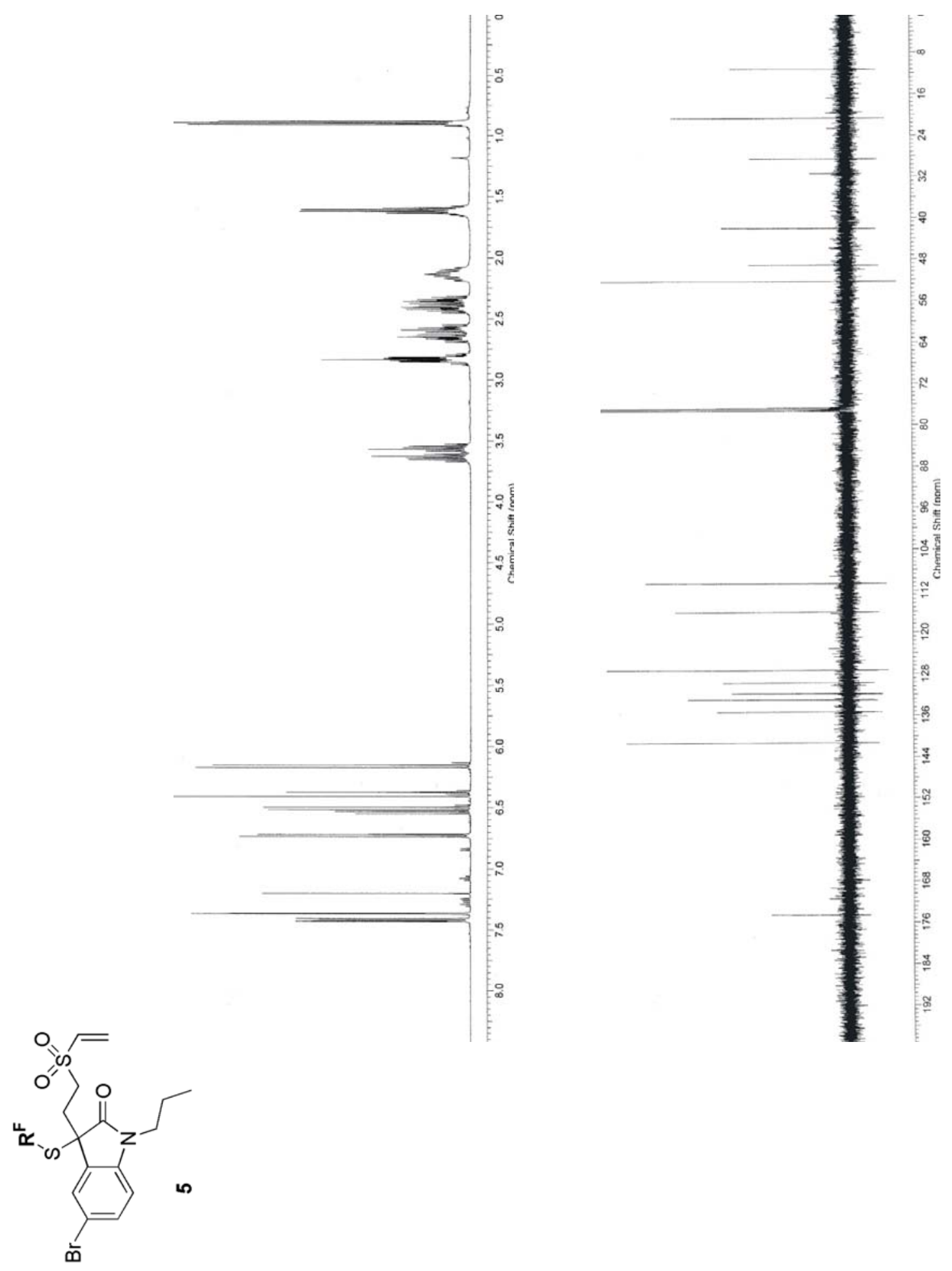

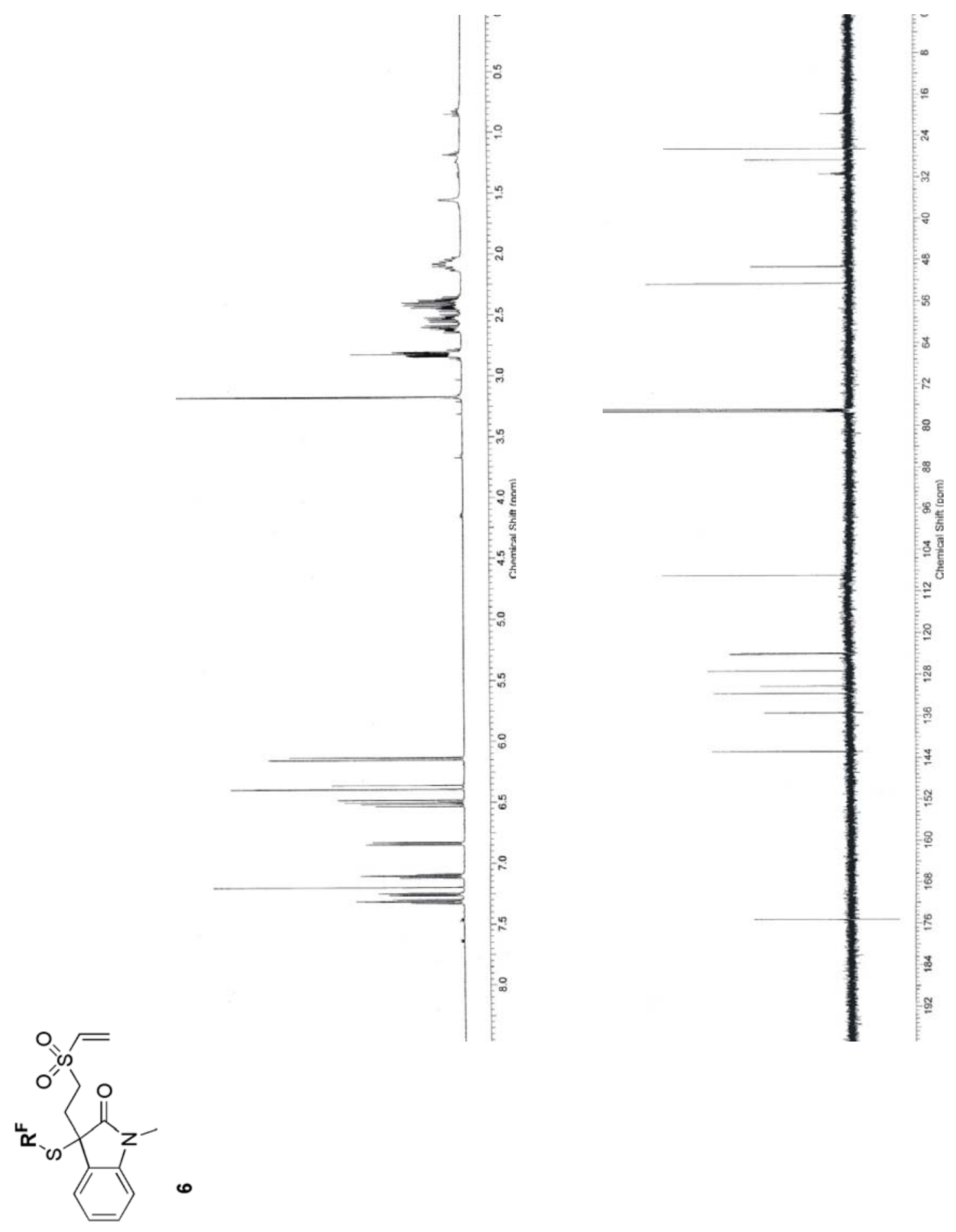

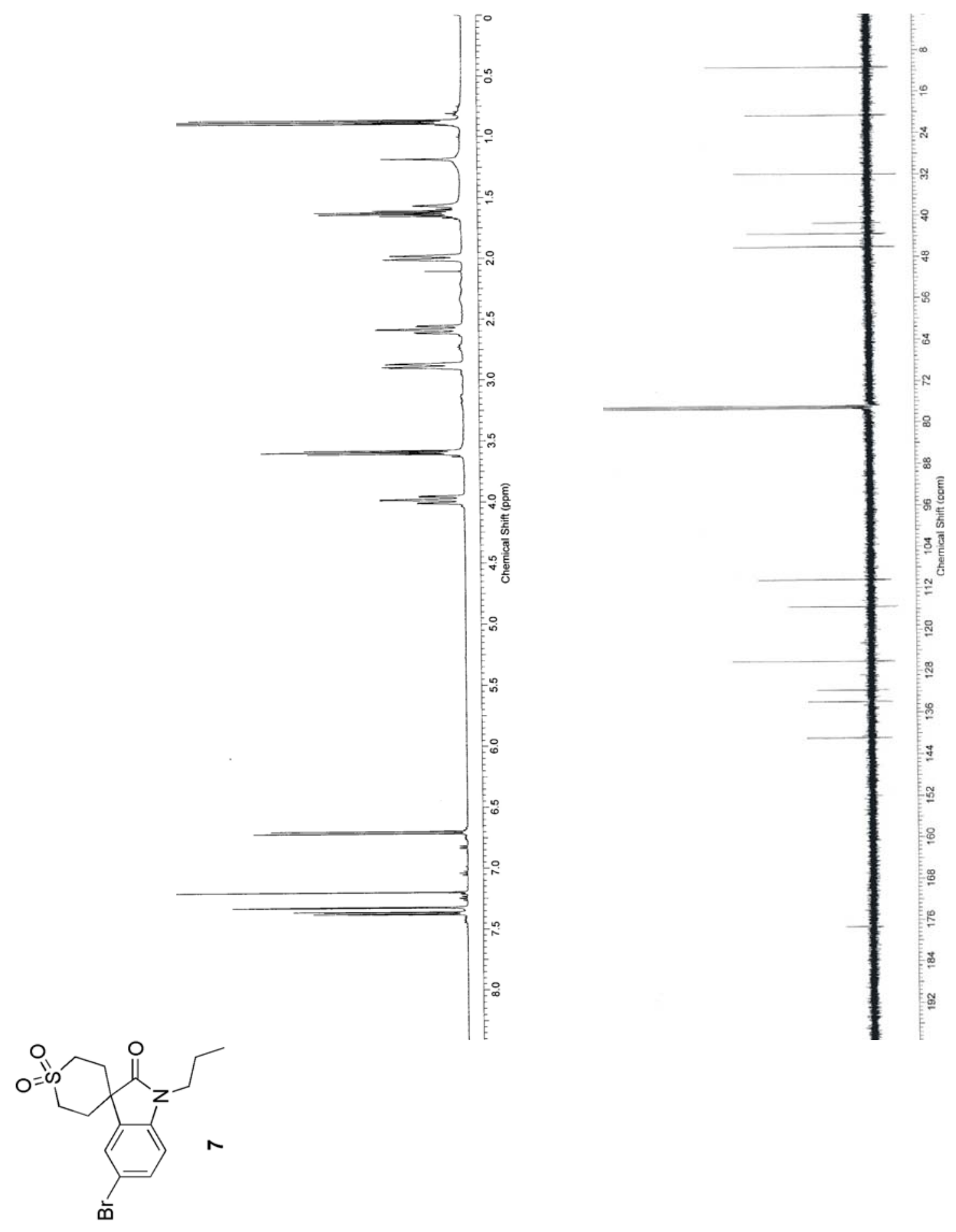

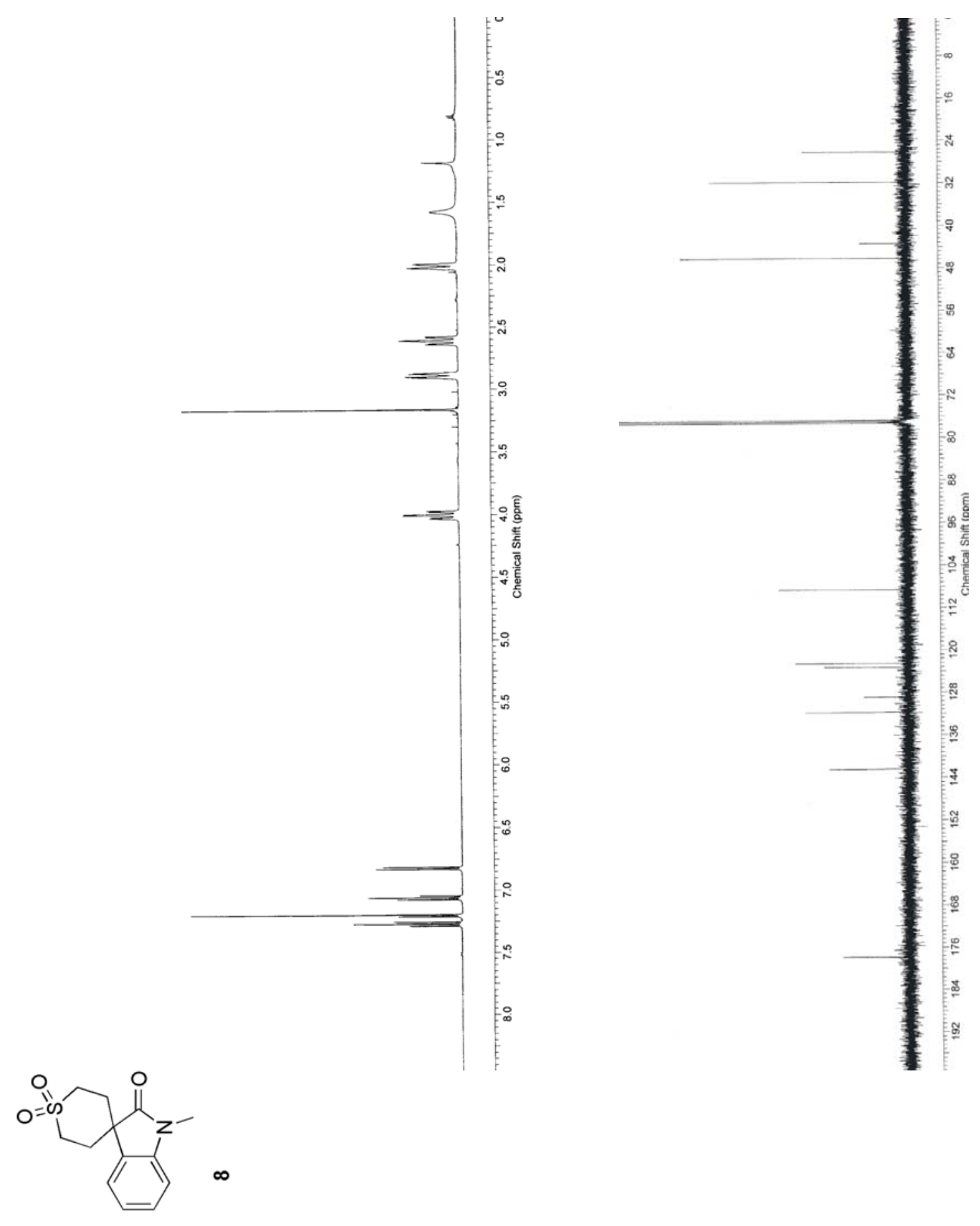


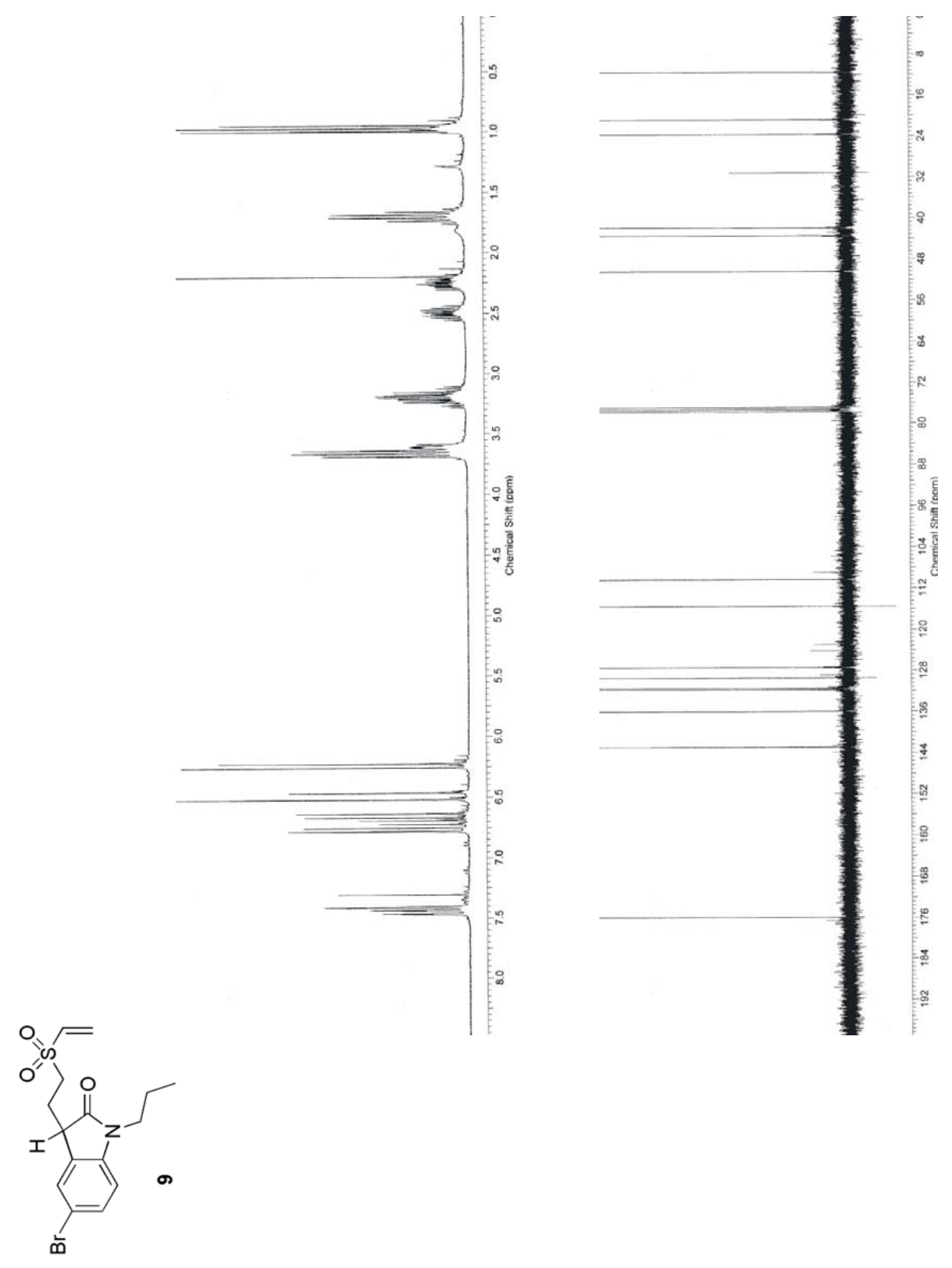



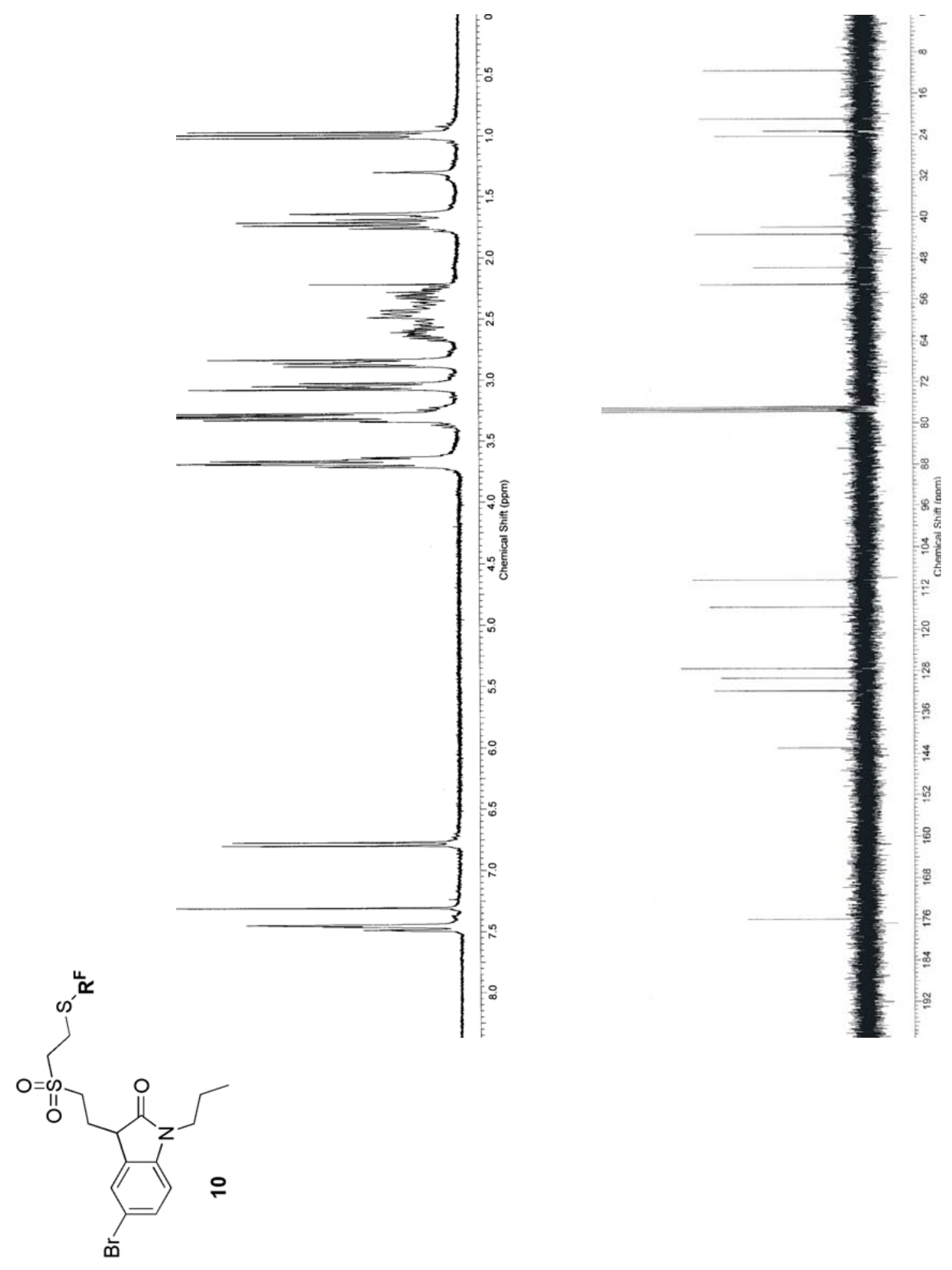

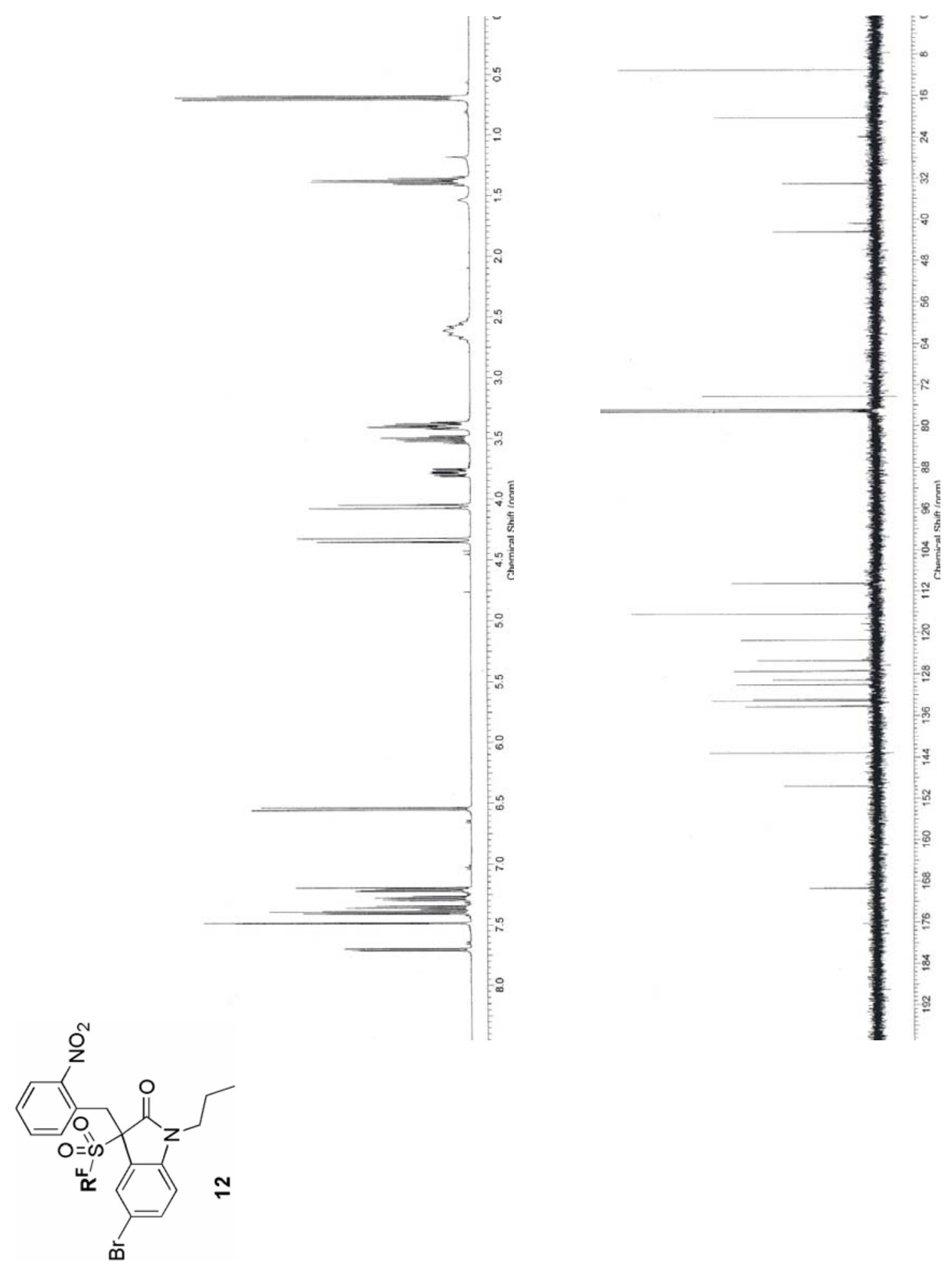

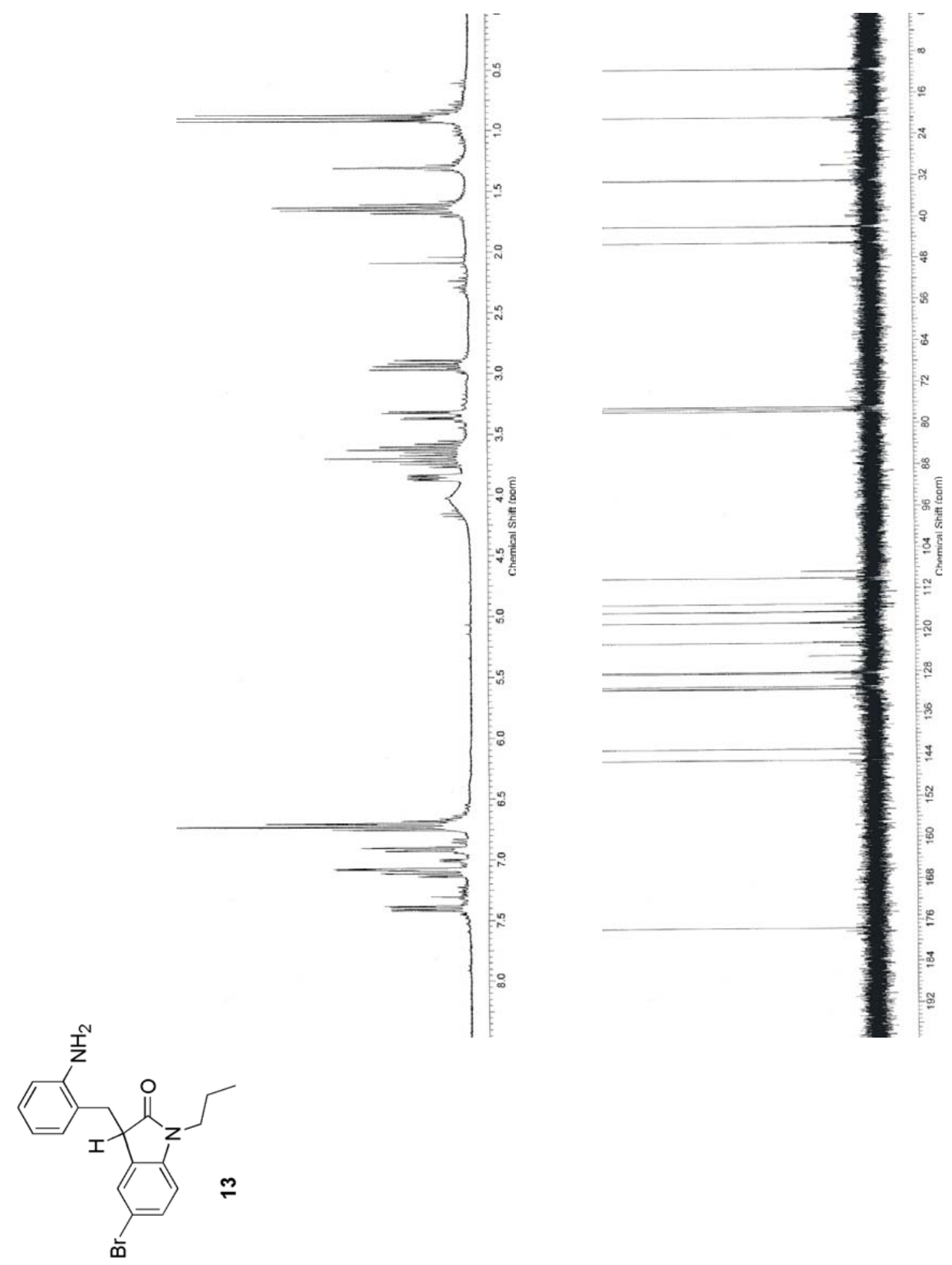

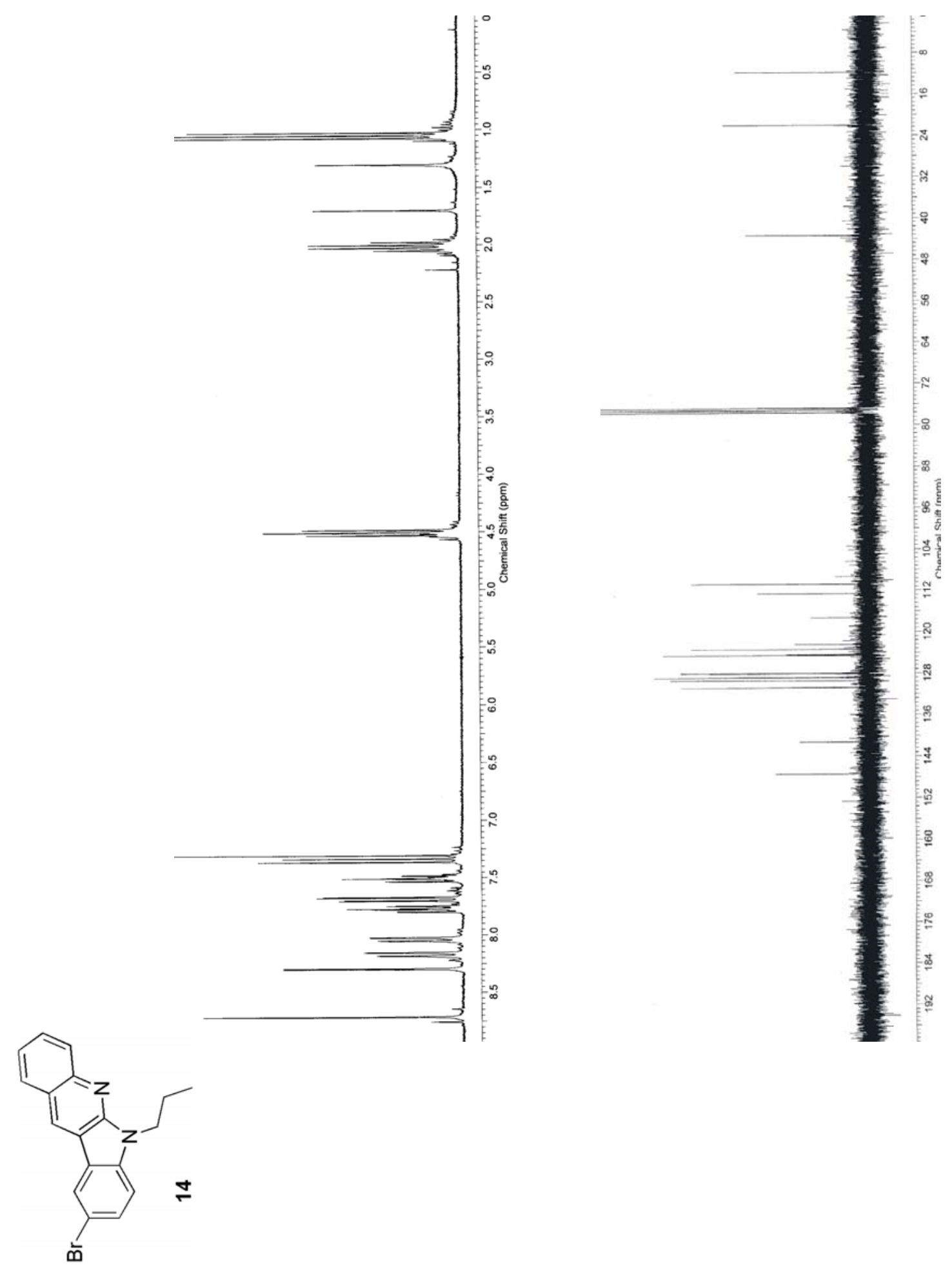

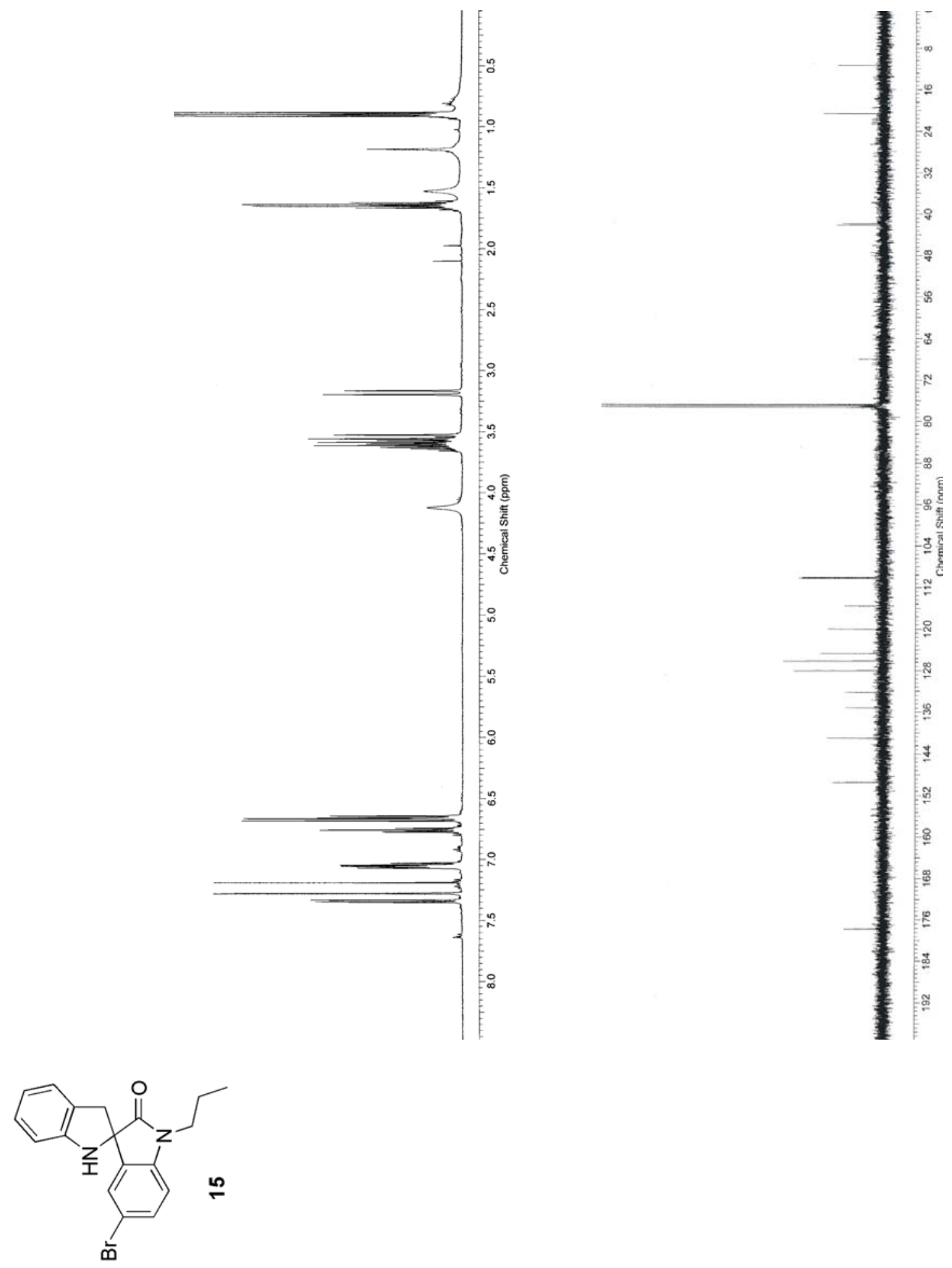

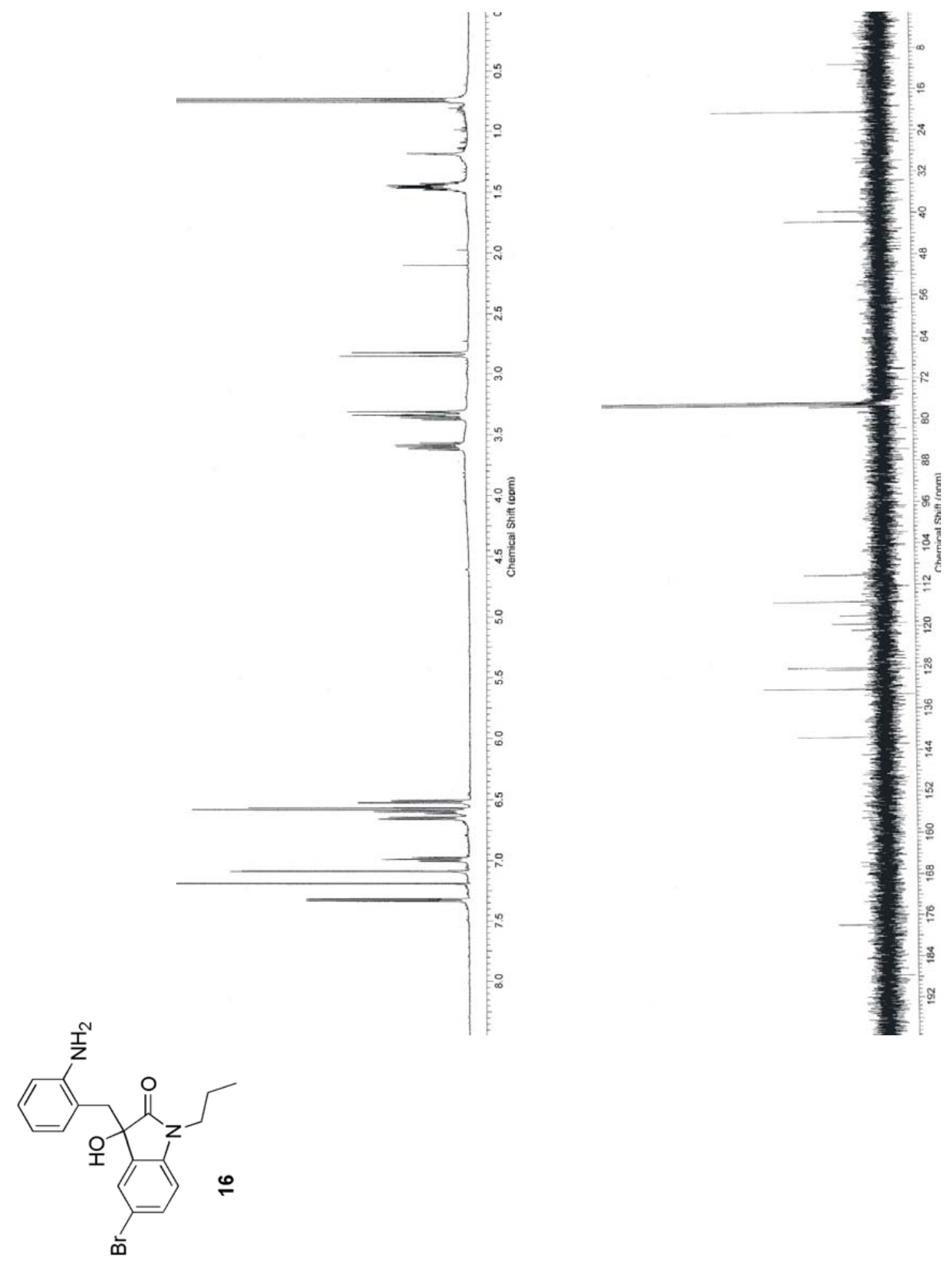

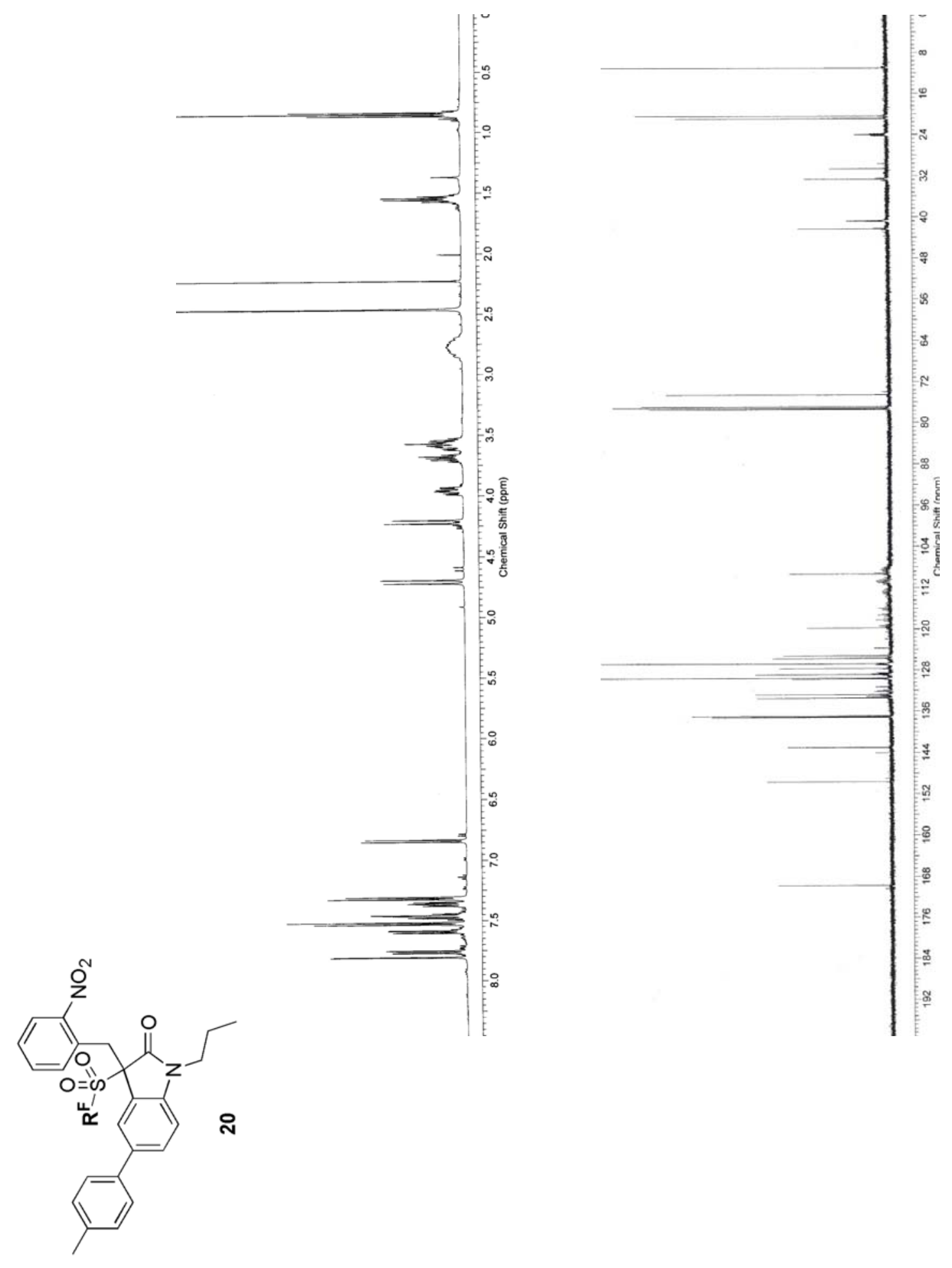

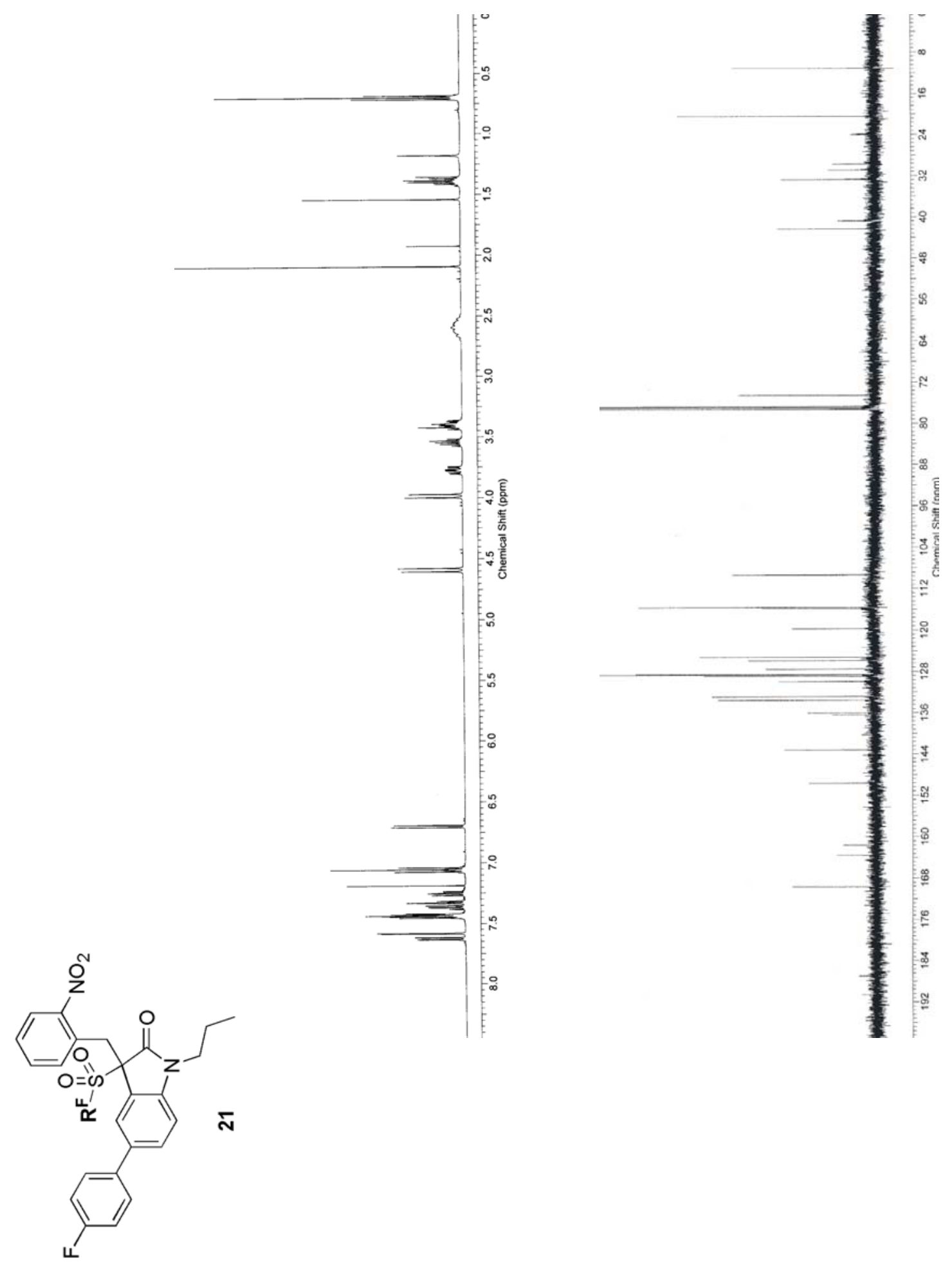

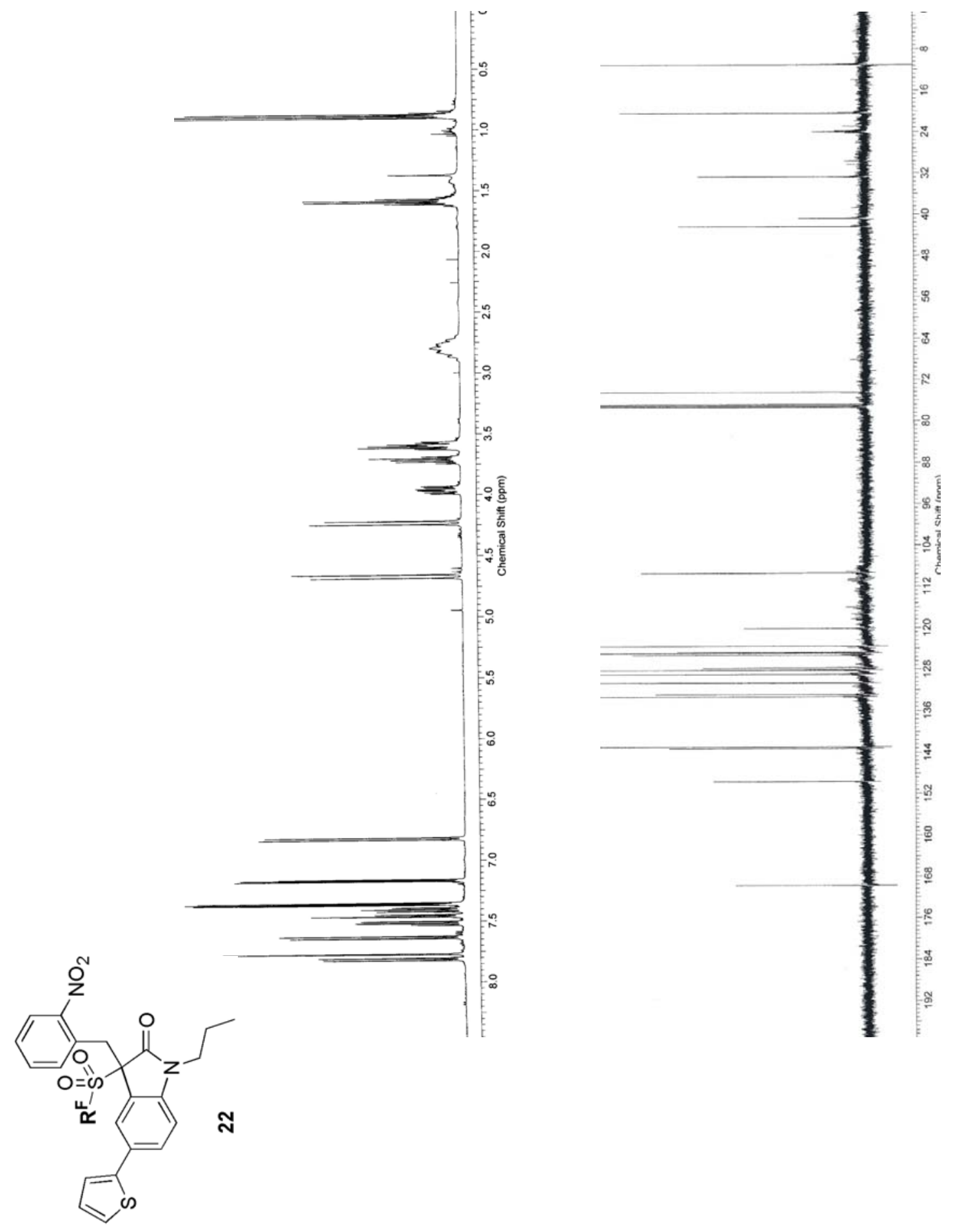

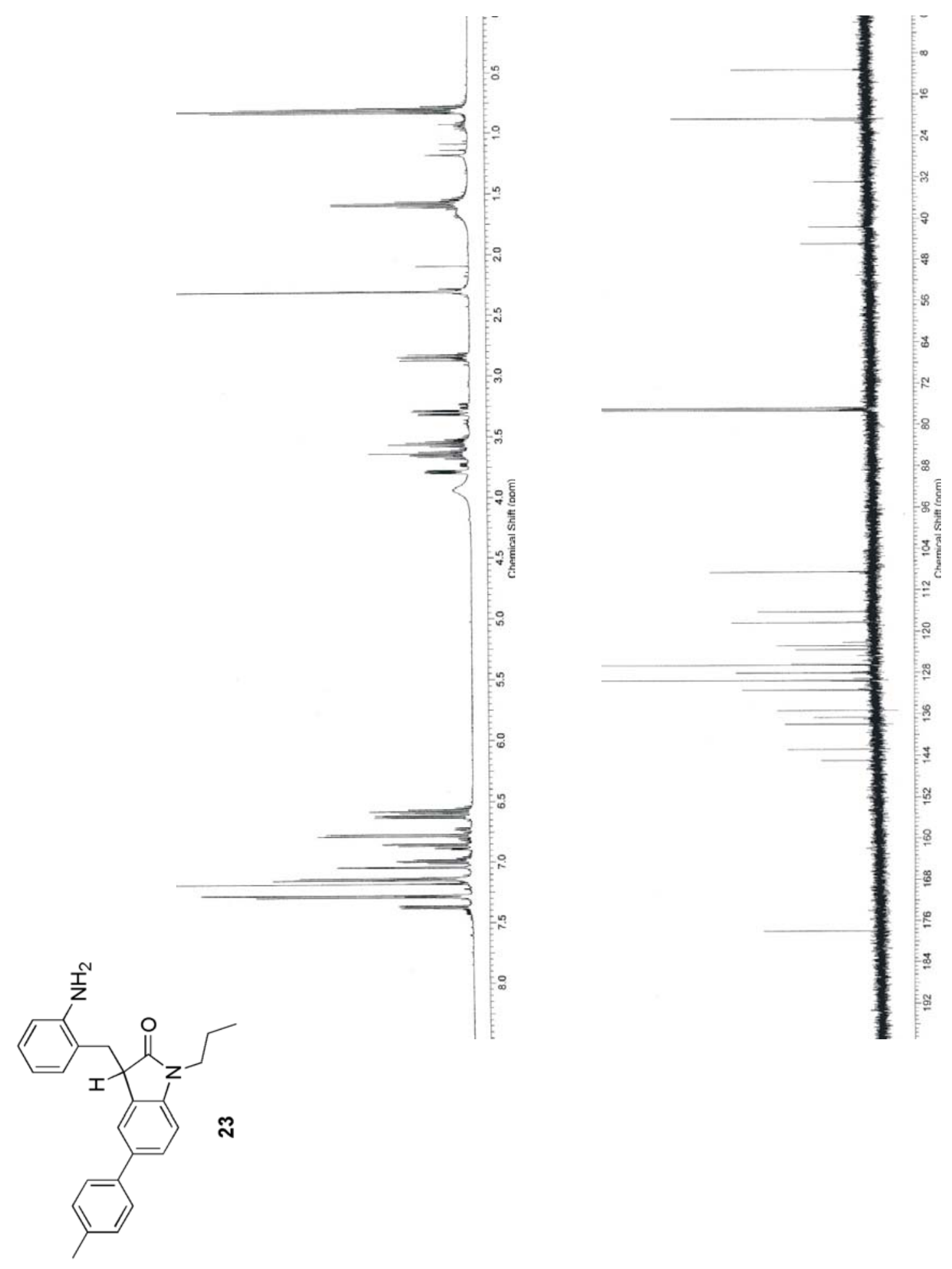

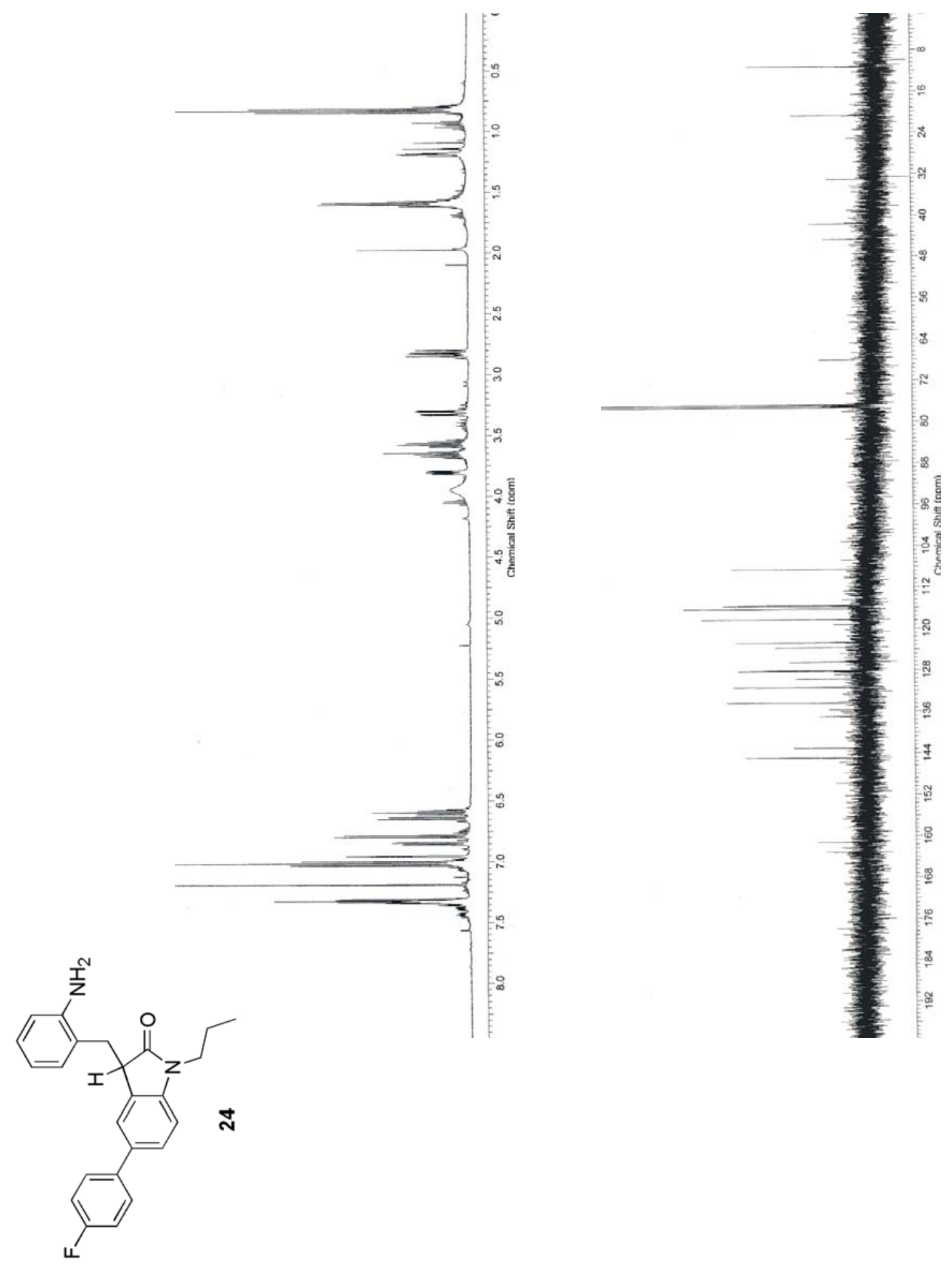

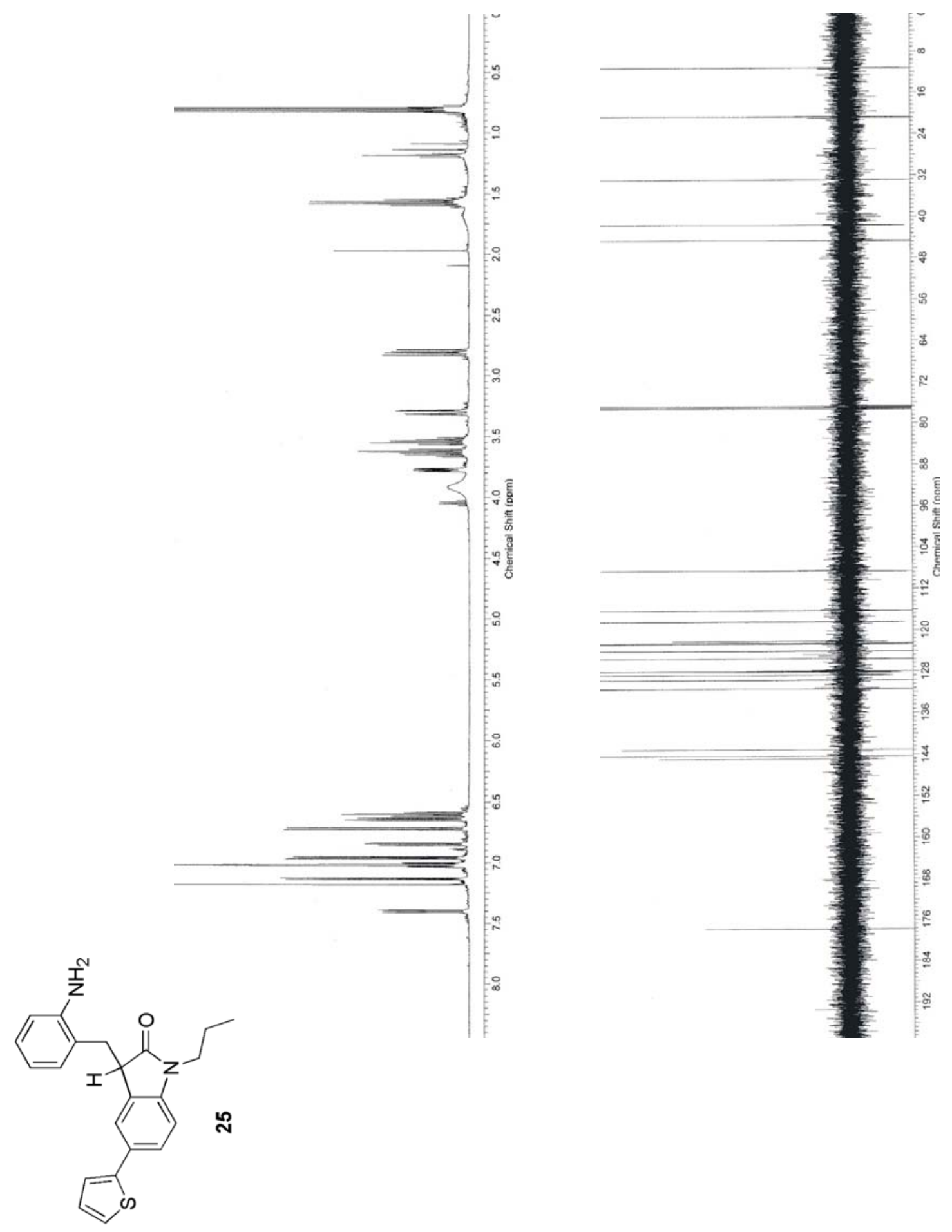

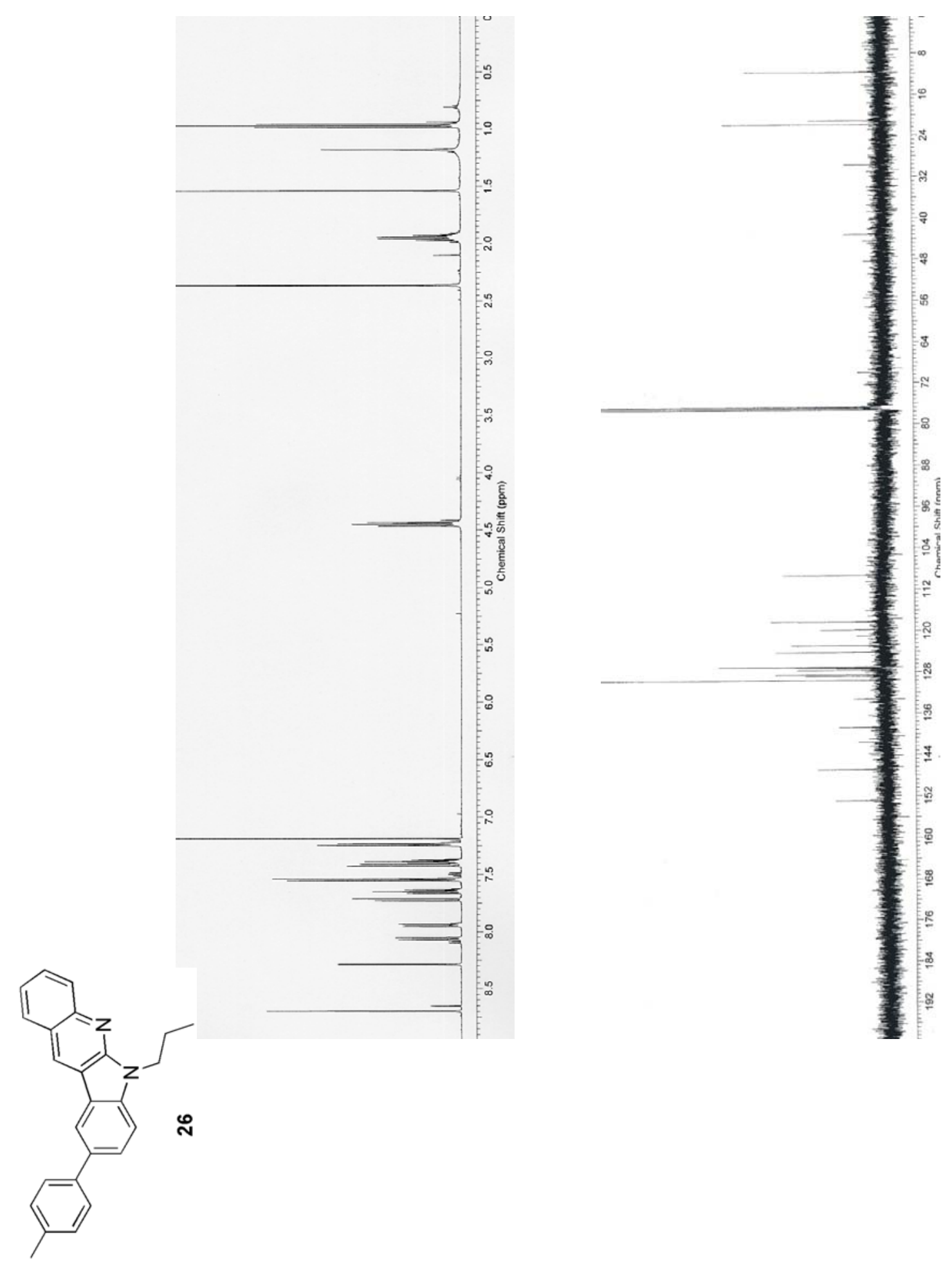

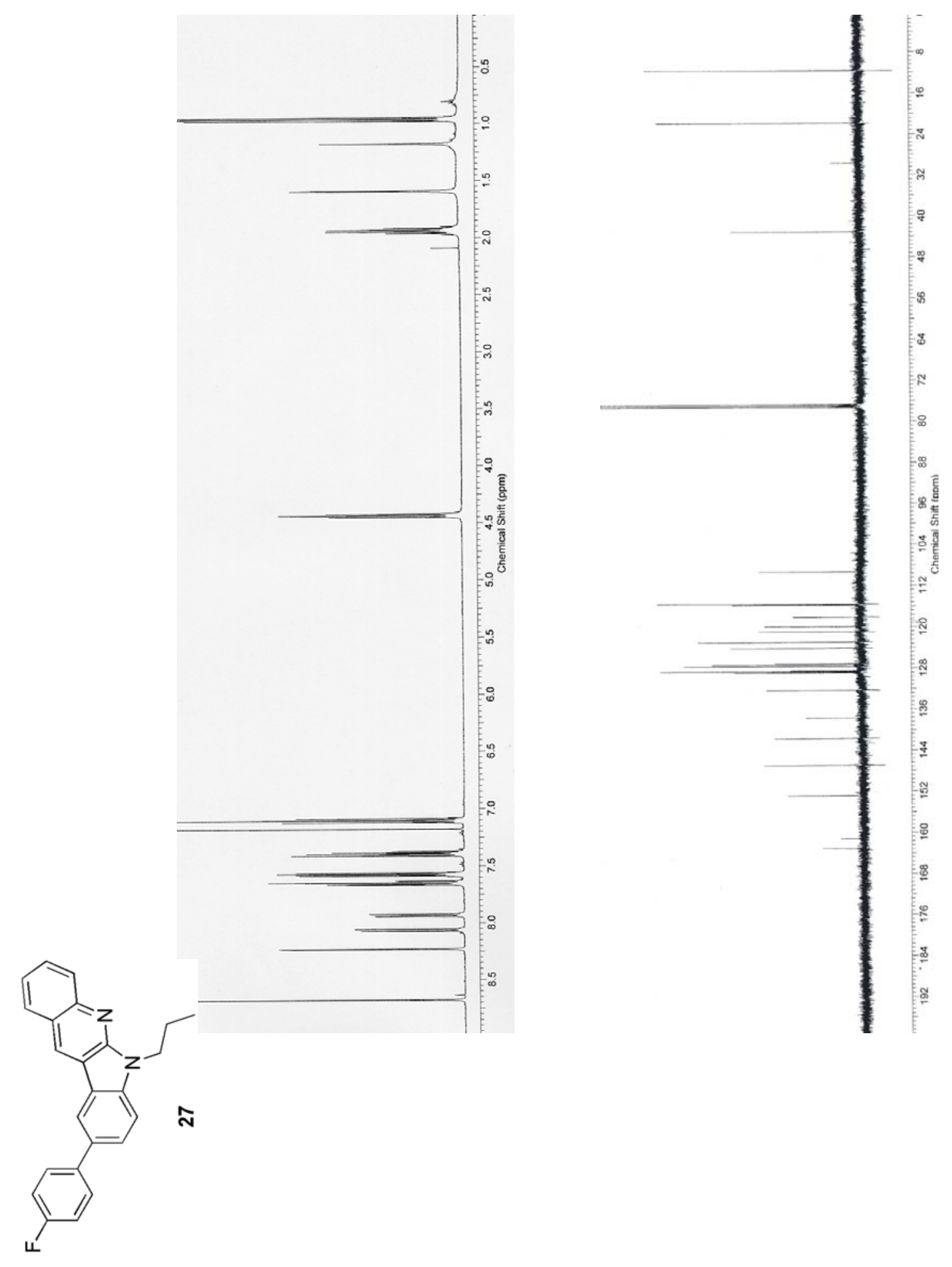

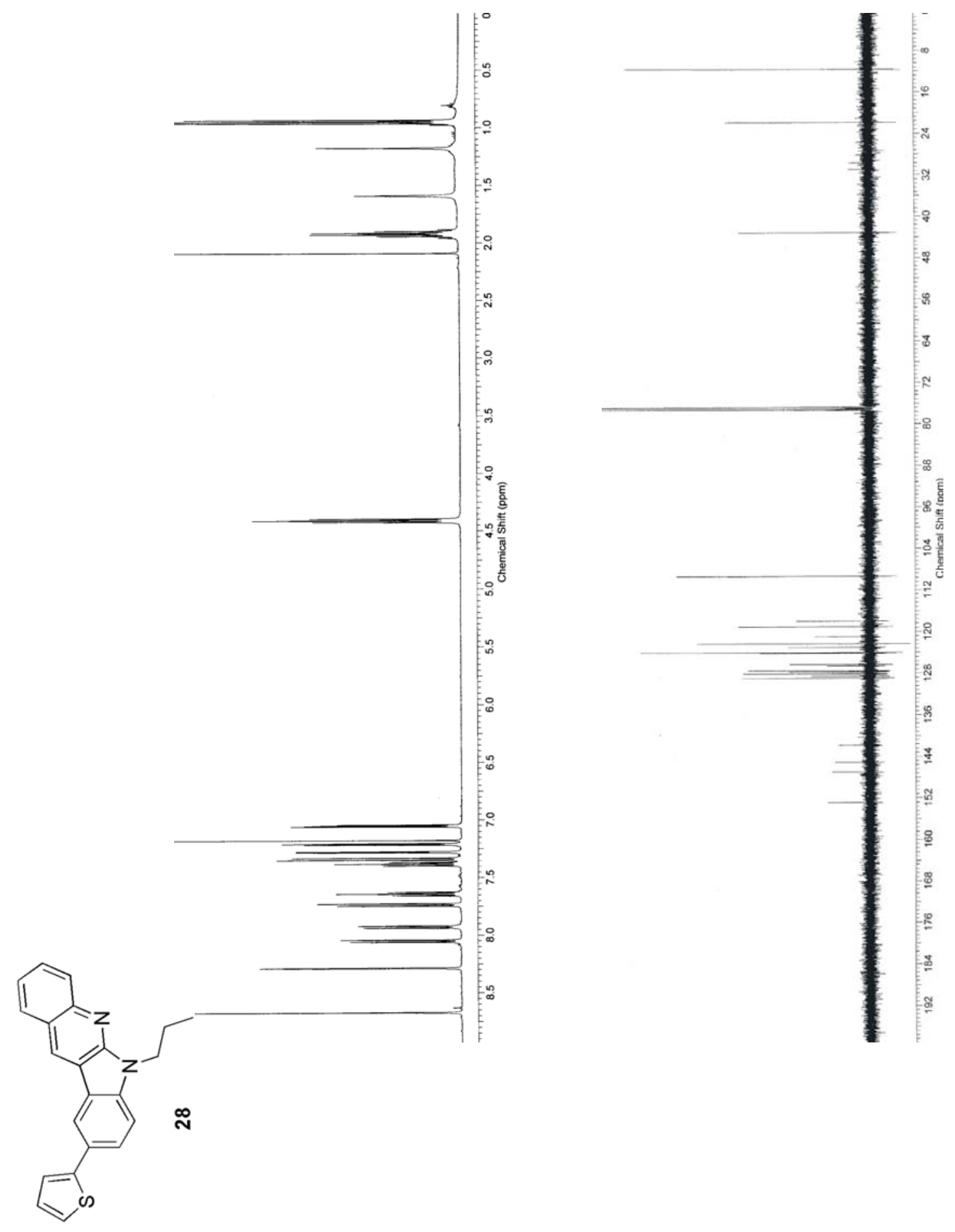

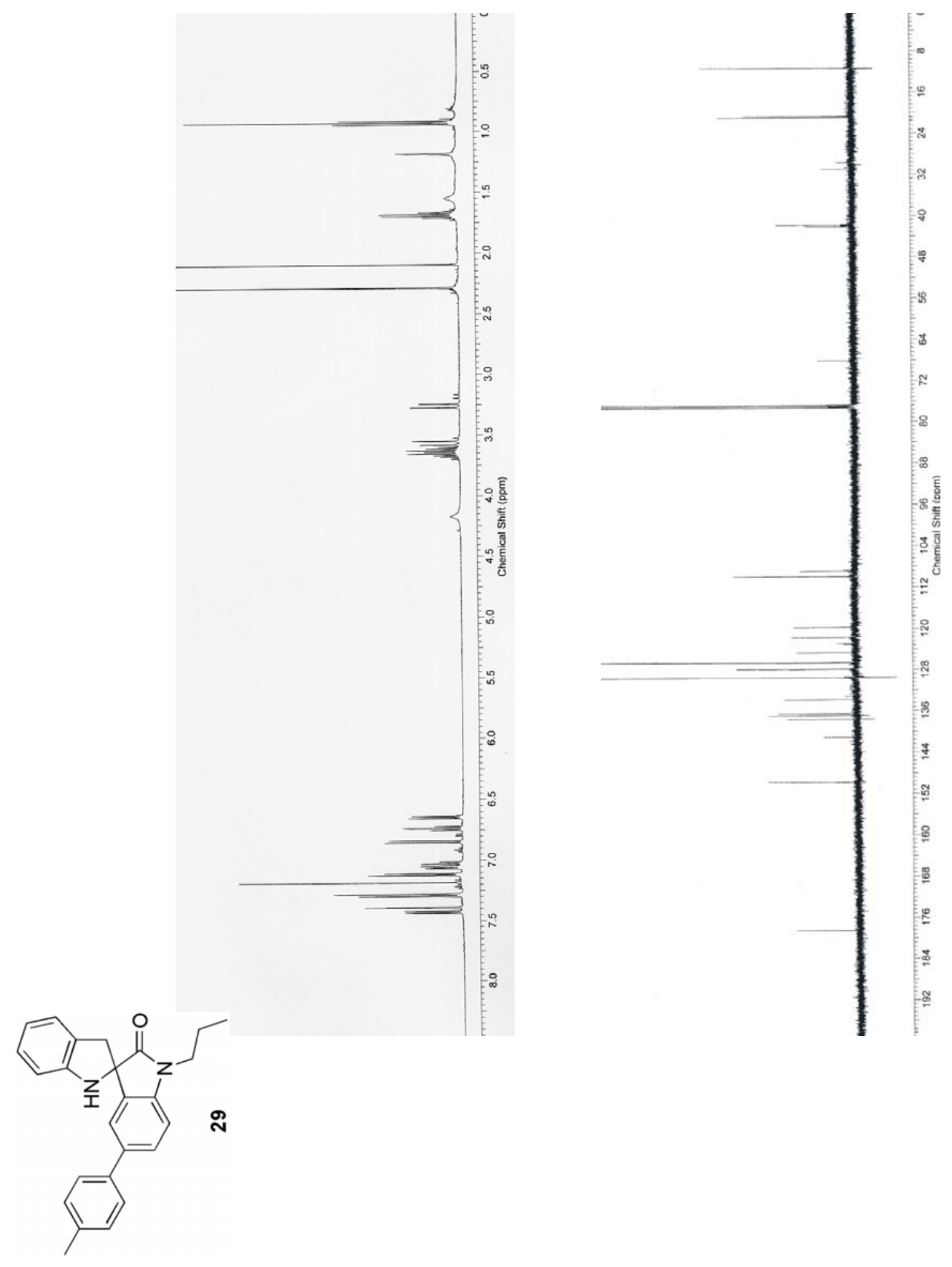

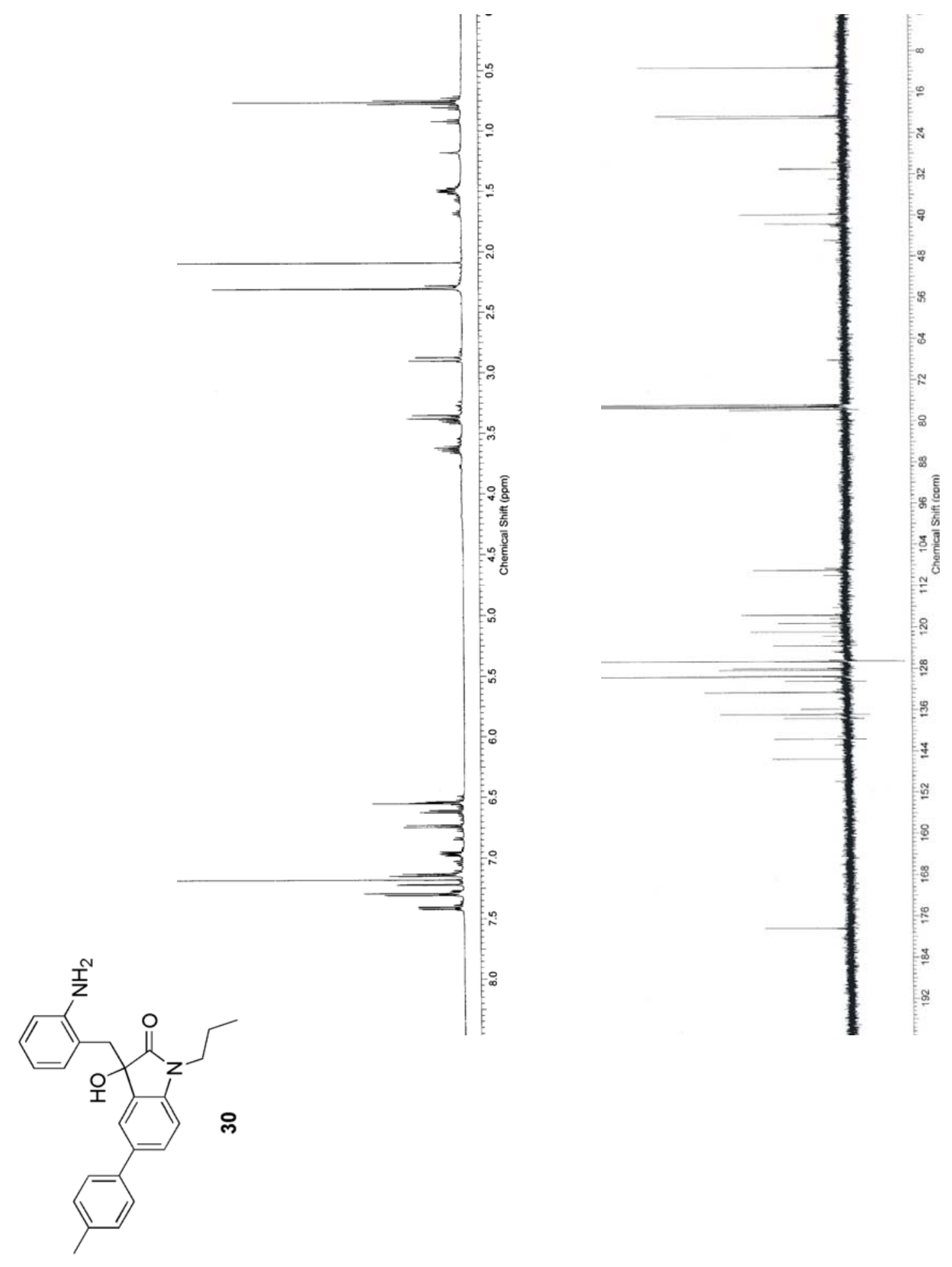